\title{
Generic matter representations in 6D supergravity theories
}

\author{
Washington Taylor and Andrew P. Turner \\ Massachusetts Institute of Technology, \\ 77 Massachusetts Avenue, Cambridge, MA, U.S.A. \\ E-mail: wati@mit.edu, apturner@mit.edu
}

ABSTRACT: In six-dimensional supergravity, there is a natural sense in which matter lying in certain representations of the gauge group is "generic," in that other "exotic" matter representations require more fine tuning. From considerations of the dimensionality of the moduli space and anomaly cancellation conditions, we find that the generic sets of matter representations are well-defined for $6 \mathrm{D}$ supergravity theories with gauge groups containing arbitrary numbers of nonabelian factors and U(1) factors. These generic matter representations also match with those that arise in the most generic F-theory constructions, both in $6 \mathrm{D}$ and in $4 \mathrm{D}$, with non-generic matter representations requiring more exotic singularity types. The analysis of generic versus exotic matter illuminates long-standing puzzles regarding F-theory models with multiple $\mathrm{U}(1)$ factors and provides a useful framework for analyzing the 6D "swampland" of apparently consistent low-energy theories that cannot be realized through known string constructions. We note also that the matter content of the standard model is generic by the criteria used here only if the global structure is $\mathrm{SU}(3)_{\mathrm{c}} \times \mathrm{SU}(2)_{\mathrm{L}} \times \mathrm{U}(1)_{Y} / \mathbb{Z}_{6}$.

Keywords: F-Theory, Field Theories in Higher Dimensions, Supergravity Models

ARXIV EPRINT: 1901.02012 


\section{Contents}

1 Introduction 1

2 Anomaly conditions in 6D supergravity 4

3 Generic matter 7

3.1 Generic matter representations for $G=\mathrm{SU}\left(N_{1}\right) \times \cdots \times \mathrm{SU}\left(N_{r}\right) \times \mathrm{U}(1)^{s}, s \leq 3 \quad 7$

3.2 Examples: generic $\mathrm{U}(1)$ and $\mathrm{SU}(2)$ matter 9

3.3 Anomaly constraints and generic matter content 11

3.4 Anomaly-equivalent exchanges and ambiguities in generic matter 12

$\begin{array}{lll}3.5 & \text { Generic matter and anomaly coefficients } & 13\end{array}$

$\begin{array}{lll}\text { 3.5.1 } & \mathrm{SU}(2) \text { generic matter and anomaly coefficients } & 14\end{array}$

$\begin{array}{lll}3.5 .2 \mathrm{U}(1) \text { generic matter and anomaly coefficients } & 16\end{array}$

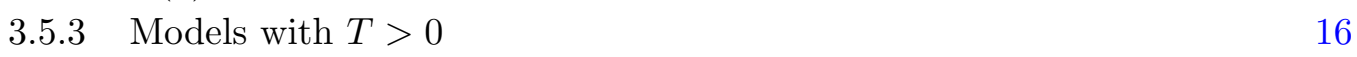

$\begin{array}{lll}3.6 & \text { Two abelian factors: } \mathrm{U}(1)^{2} & 19\end{array}$

3.6.1 Choices of generic matter spectra for $\mathrm{U}(1) \times \mathrm{U}(1)$ theories 20

3.6.2 Generic $\mathrm{U}(1) \times \mathrm{U}(1)$ matter from Higgsing nonabelian theories 22

3.6.3 Generic $\mathrm{U}(1) \times \mathrm{U}(1)$ matter at small $b, T=0 \quad 23$

3.7 Three abelian factors: $\mathrm{U}(1)^{3} \quad 23$

3.8 Generic matter with more than three U(1) factors 27

$\begin{array}{ll}3.9 & \text { Other nonabelian factors } \\ \end{array}$

4 Generic matter in F-theory and the string swampland $\quad 29$

4.1 Tate and Weierstrass models for $\mathrm{SU}(N)$ gauge groups 29

$4.2 \mathrm{U}(1)$ matter in F-theory 32

4.2.1 One U(1) factor 32

4.2.2 Two U(1) factors 33

4.2.3 Three or more U(1) factors 33

4.3 Generic matter, string universality, and the swampland 34

4.3.1 Structure of 6D supergravity theories 35

4.3.2 Positivity cone and anomaly lattice related swampland issues $\quad 36$

$\begin{array}{lll}\text { 4.3.3 The swampland for generic matter } & 36\end{array}$

4.3.4 Exotic matter and the 6D swampland $\quad 40$

$\begin{array}{lll}4.3 .5 & \text { Swampland summary } & 40\end{array}$

5 Generic matter with global gauge group structure and 4D physics $\quad 40$

5.1 Generic matter and the global structure of the gauge group 40

5.2 Generic matter in 4D supergravity theories and F-theory models 43

5.3 Generic matter and the standard model 43

$\begin{array}{lll}6 & \text { Conclusions } & 46\end{array}$ 
A Generic matter for $\mathrm{SU}\left(N_{1}\right) \times \cdots \times \mathrm{SU}\left(N_{r}\right) \times \mathrm{U}(1)^{3}$

$\begin{array}{lll}\text { A.1 } & \mathrm{SU}(N) & 48\end{array}$

$\begin{array}{lll}\text { A.2 } & \mathrm{U}(1)^{3} & 52\end{array}$

A.3 $\mathrm{SU}(N) \times \mathrm{U}(1)^{3}$

A.4 $\mathrm{SU}\left(N_{1}\right) \times \mathrm{SU}\left(N_{2}\right) \times \mathrm{U}(1)^{3}$

A.5 $\quad \mathrm{SU}\left(N_{1}\right) \times \cdots \times \mathrm{SU}\left(N_{r}\right) \times \mathrm{U}(1)^{3}$

B Generic SU(2) matter at small $b, T>0 \quad 54$

C Generic matter spectra for $G=\mathrm{U}(1)^{2} \quad 56$

\section{Introduction}

In principle, it seems possible to define low-energy field theories in various dimensions with a given gauge group $G$ and matter that can live in essentially any representation of the group $G$. For example, one could imagine a quantum field theory with a U(1) gauge group and elementary particles associated with excitations of matter fields that transform with fairly arbitrary combinations of charges $q_{i}$, or a QFT with an $\mathrm{SU}(2)$ gauge group and elementary particles transforming in a representation of $\mathrm{SU}(2)$ with arbitrarily large dimension. From this point of view, it may seem surprising that the standard model of particle physics that we observe in nature has only very simple types of charges under the electromagnetic, weak, and strong forces.

From the point of view of string theory, however, the most natural constructions tend to lead to fairly simple representations, such as the fundamental, adjoint, and two-index antisymmetric and symmetric representations of $\mathrm{SU}(N)$. There are also constraints from quantum consistency conditions such as anomalies that limit the set of possible matter representations, particularly in higher-dimensional theories with more symmetry.

In this paper we consider the question of whether some matter representations are more generic than others in a concrete context where the notion of "generic" can be given a quantitative meaning. In particular, we consider matter spectra in six-dimensional $\mathcal{N}=(1,0)$ supergravity theories. Six is the largest dimension in which a supersymmetric theory with a gauge group $G$ can have matter in any representation other than the adjoint. Six dimensional supergravity also has very strong conditions for the cancellation of gauge, gravitational, and mixed gauge-gravitational anomalies [1,2]; these anomaly cancellation conditions, for example, restrict the set of possible matter representations of nonabelian gauge groups to a finite set (at least when the number of tensor multiplets does not exceed 8) [3, 4], although they do not place a bound on U(1) charges [5]. Perhaps most importantly, however, the space of six-dimensional supergravity theories consists of a set 
of interconnected ${ }^{1}$ branches, where each branch constitutes a moduli space of fixed dimensionality. By comparing the dimensionality of a branch of moduli space having, for example, a $\mathrm{U}(1)$ gauge group and elementary charges $q=1,2$ with a branch having a $\mathrm{U}(1)$ gauge group and elementary charges $q=1,2,3$ and the same anomaly coefficients, we can say that the former set of charges is more generic because the dimensionality of the first branch of the moduli space is larger, so that the set of theories with charges $q=3$ requires more fine tuning of the theory.

A precise definition of generic matter representations requires consideration of how the matter content depends on the anomaly structure of the theory. After a brief review of $6 \mathrm{D}$ anomaly conditions in section 2, we consider the notion of generic matter in $6 \mathrm{D}$ from the points of view of moduli space dimension and anomalies in section 3, and in section 4 we describe generic matter in F-theory and discuss how generic matter illuminates questions about the $6 \mathrm{D}$ string landscape and swampland. By analyzing the dimensionality of various branches of the $6 \mathrm{D}$ supergravity moduli space with different charge content, we find that in many situations generic matter representations can be defined as those on the branch of moduli space of greatest dimension, when the other discrete parameters of the theory are taken to be fixed. In the simplest cases, with at most one nonabelian gauge factor and one abelian U(1) factor, this notion matches well with the anomaly cancellation conditions, in the sense that the number of distinct generic matter representations matches the number of anomaly cancellation conditions, so that the generic matter content in a given theory is determined as a unique solution of the anomaly cancellation equations restricted to the generic matter fields; these equations are fixed in terms of the discrete parameters characterizing the number of tensor multiplets, the gauge group, and the anomaly coefficients. Furthermore, we find that the types of generic matter singled out by moduli space dimensions and anomaly cancellation conditions match precisely with the types of matter constructed in the most generic F-theory models with a given gauge group. As examples, in the case of a $\mathrm{U}(1)$ gauge group, all of these considerations point to $q=1,2$ as the generic matter charges, and in the case of a single $\mathrm{SU}(2)$ gauge group, generic matter is in the fundamental and adjoint representations. As the gauge group becomes more complicated, however, generic matter becomes less uniquely defined; in particular, we find that when there are multiple $\mathrm{U}(1)$ factors, the number of anomaly-inequivalent charge combinations associated with generic matter becomes larger than the number of anomaly cancellation conditions, so that in the "generic" models of maximal moduli space dimension, only a subset of all the generic charge combinations are realized.

While most of the considerations in this paper are based on $6 \mathrm{D}$ supergravity, the fact that the generic matter representations identified in 6D match with those constructed by generic F-theory models suggests that the same or similar notions of generic matter may be relevant in four dimensions. In section 5, we discuss how the framework of this

\footnotetext{
${ }^{1}$ It is not proven that all consistent $6 \mathrm{D}$ supergravity theories lie on a single connected moduli space. This is true of all conventional F-theory models, since all elliptic Calabi-Yau threefolds are connected through various topological transitions, as discussed in, e.g., [4]. There may, however, be other branches associated with "frozen phase" F-theory models that are not connected in the same way with the set of conventional F-theory models [6].
} 
paper illuminates various questions relevant to both $6 \mathrm{D}$ and $4 \mathrm{D}$ supergravity, including the possible relevance of this kind of analysis to the standard model. In section 6 , we make some concluding remarks and describe some further directions for related investigations.

We close this introduction with a brief discussion of the term "generic" and the sense in which the matter representations we define as generic here are natural from a physics perspective. The word "generic" is a somewhat dangerous term, which is used in many different ways by physicists. We use this term here in a very specific way. In particular, we define generic matter representations to be those that arise in 6D supergravity theories with a fixed gauge group and number of tensor multiplets on the moduli space branch of highest dimensionality given relatively small anomaly coefficients. We feel this term is appropriate and useful in this context because it matches structure associated with anomaly cancellation conditions and the geometry of F-theory. The idea that the generic matter fields are those on the largest-dimensional component of moduli space essentially captures the idea that other matter representations involve some fine tuning, in the same sense that an arbitrary point $x_{i}$ in a 37-dimensional space is more generic than one that satisfies a system of 16 algebraic equations $f_{j}(x)=0$. An important aspect of this definition is, however, that we have fixed the gauge group of the theory, and the generic matter fields are defined only with respect to this certain choice of gauge group. More broadly speaking, some gauge groups themselves may be non-generic from the point of view of string compactifications. For example, in 6D supergravity theories coming from F-theory, if we fix the number of tensor multiplets and the string charge lattice - associated with a given choice of elliptic Calabi-Yau threefold for the F-theory geometry - the only generic gauge groups are those associated with non-Higgsable clusters [7], which typically support no or minimal matter. The generic matter fields for a given gauge group $G$ that we describe here thus address what is in some sense a second-order question, where the gauge group $G$ itself may require some fine-tuning of the geometry and we inquire about the expectations for generic matter given that first-order fine tuning. In terms of the full $6 \mathrm{D}$ supergravity moduli space, the branches with a tuned gauge group are themselves of smaller dimensionality than the generic branches with only (supersymmetrically) non-Higgsable gauge group factors. How this notion of genericity for either gauge groups or matter applies for four-dimensional theories, from F-theory or otherwise, is much less clear, although at the geometric level there are certain gauge groups that are similarly generic associated with $4 \mathrm{D}$ non-Higgsable clusters [8]; we discuss questions of generic matter for $4 \mathrm{D}$ theories a bit further in section 5.2.

Finally, another important issue that we have not addressed here is the role of strongly coupled matter, associated in the F-theory picture with $(4,6)$ non-Kodaira type singularities at codimension two in the base of the elliptic fibration. These are associated with superconformal field theories (SCFTs) coupled to the gravity theory giving rise to "conformal matter" [9-12]. Studies of the global structure of the space of 4D F-theory models show that such strongly coupled conformal matter appears quite broadly throughout the string landscape $[13,14]$, and may in fact provide a natural mechanism for realizing the standard model within 4D F-theory [15, 16]. Incorporating strongly coupled conformal matter into our understanding of generic gauge groups and matter in a systematic way presents promising avenues for further research. 


\section{Anomaly conditions in 6D supergravity}

We write the gauge group in the form

$$
G=\prod_{\kappa=1}^{r} G_{\kappa} \times \prod_{i=1}^{s} \mathrm{U}(1)_{i},
$$

where the $G_{\kappa}$ are simple nonabelian gauge group factors. In general, we will use lowercase Greek letters to index simple nonabelian gauge group factors, and lowercase Roman letters to index $\mathrm{U}(1)$ factors. The numbers of simple nonabelian and abelian gauge group factors are respectively denoted $r$ and $s$. Hypermultiplets in the irreducible representation $R$ transform in the representation $R_{\kappa}$ of $G_{\kappa}$ and have $\mathrm{U}(1)_{i}$ charge $q_{R, i}$. That is, we indicate the individual factors of a representation via

$$
R=\bigotimes_{\kappa=1}^{r} R_{\kappa} \otimes \bigotimes_{i=1}^{s} q_{R, i}
$$

Note that a hypermultiplet we refer to as being in representation $R$ actually contains fields transforming in both $R$ and $\bar{R}$, as the matter representations in a $6 \mathrm{D}$ theory must be quaternionic. For representations $R$ that are themselves quaternionic, we may have "halfhypermultiplets" with half the field content, only transforming in the representation $R$.

The dimension $d_{R}$ of the representation $R$ is given by

$$
d_{R}=\prod_{\kappa} d_{R_{\kappa}},
$$

where $d_{R_{\kappa}}$ is the dimension of the representation $R_{\kappa}$ of the gauge group factor $G_{\kappa}$. We use $d_{R}^{\kappa}$ and $d_{R}^{\kappa \mu}$ to respectively denote the number of $G_{\kappa}$ and $G_{\kappa} \times G_{\mu}$ representations $R_{\kappa}$ and $R_{\kappa} \otimes R_{\mu}$ in $R$, given by

$$
d_{R}^{\kappa}=\prod_{\lambda \neq \kappa} d_{R_{\lambda}}, \quad d_{R}^{\kappa \mu}=\prod_{\lambda \neq \kappa, \mu} d_{R_{\lambda}} .
$$

We use $x$ with various superscripts and subscripts to indicate the number of hypermultiplets transforming in a given representation. Subscripts indicate representations, and superscripts indicate to which gauge factor these representations belong. Thus, for example, $x_{R}^{\kappa}$ indicates the number of hypermultiplets transforming in the representation $R$ of the factor $G_{\kappa}, x_{R, S}^{\kappa, \mu}$ indicates the number of hypermultiplets transforming in the representation $R \otimes S$ of the product $G_{\kappa} \times G_{\mu}$, and $x_{q, r, s, t}^{i, j, k, \ell}$ indicates the number of hypermultiplets transforming in the representation $q \otimes r \otimes s \otimes t$ of the abelian product $\mathrm{U}(1)_{i} \times \mathrm{U}(1)_{j} \times \mathrm{U}(1)_{k} \times \mathrm{U}(1)_{\ell}$. For ease of notation, we will omit the superscripts when they would include all factors of the gauge group, so that, for example, $x_{q, r}:=x_{q, r}^{1,2}$ for $G=\mathrm{U}(1)^{2}$. Because we use $R_{\kappa}$ to explicitly indicate a representation of factor $G_{\kappa}$ and $q_{i}$ to indicate a charge of factor $\mathrm{U}(1)_{i}$, we will also use a compressed notation that omits the superscripts when they can be inferred from the subscripts, e.g., $x_{R_{\kappa}, R_{\mu}}:=x_{R_{\kappa}, R_{\mu}}^{\kappa, \mu}$.

These multiplicities are defined so as to account for the dimensions of the representations of the other group factors in every representation $\tilde{R}$ of $G$ that contains the relevant 
representation, i.e.,

$$
x_{R_{\kappa}}=\sum_{\tilde{R} \supset R_{\kappa}} x_{\tilde{R}} d_{\tilde{R}}^{\kappa}
$$

Similarly, we have

$$
\begin{array}{r}
x_{R_{\kappa}, R_{\mu}}=\sum x_{\tilde{R}} d_{\tilde{R}}^{\kappa \mu}, \\
x_{R_{\kappa}, q_{i}}=\sum x_{\tilde{R}} x_{\tilde{R}} d_{\tilde{R}}^{\kappa}, \\
x_{R_{\kappa}, q_{i}, q_{j}}=\sum x_{\tilde{R}} x_{\tilde{R}} d_{\tilde{R}}^{\kappa}, \\
\tilde{R} \supset R_{\kappa} \otimes q_{i} \otimes q_{j} \\
x_{q_{i}, q_{j}, q_{k}, q_{\ell}}=\sum x_{\tilde{R}} d_{\tilde{R}} . \\
\tilde{R} \supset q_{i} \otimes q_{j} \otimes q_{k} \otimes q_{\ell}
\end{array}
$$

It is important to note that these multiplicities are related to one another when indices are duplicated, e.g.,

$$
x_{q, r, s, t}^{i, i, k, \ell}=\delta_{q r} x_{q, s, t}^{i, k, \ell}, \quad \text { (no summation) } .
$$

The numbers of massless vector multiplets and hypermultiplets are denoted $V$ and $H$, respectively, and are given by

$$
V=V_{\mathrm{NA}}+V_{\mathrm{A}}=\sum_{\kappa} d_{\mathrm{Adj}_{\kappa}}+s, \quad H=\sum_{R} x_{R} d_{R},
$$

where $V_{\mathrm{NA}}$ is the number of nonabelian vector multiplets in the theory, $V_{\mathrm{A}}=s$ is the number of abelian vector multiplets, and $\mathrm{Adj}_{\kappa}$ is the adjoint representation of the gauge group factor $G_{\kappa}$. The number of tensor multiplets is denoted by $T$.

We will use tr to denote the trace in the fundamental representation and $\operatorname{tr}_{R}$ to denote the trace in an arbitrary representation $R$.

The terms in the anomaly polynomial with no abelian field strength factors give rise to the conditions for cancellation of gravitational, gauge, and mixed gauge-gravitational anomalies for the nonabelian factors, which in the notation of [4] are

$$
\begin{aligned}
273 & =H-V+29 T \\
a \cdot a & =9-T \\
a \cdot b_{\kappa} & =-\frac{1}{6} \lambda_{\kappa}\left(\sum_{R_{\kappa}} x_{R_{\kappa}} A_{R_{\kappa}}-A_{\mathrm{Adj}_{\kappa}}\right), \\
0 & =\sum_{R_{\kappa}} x_{R_{\kappa}} B_{R_{\kappa}}-B_{\mathrm{Adj}_{\kappa}}, \\
b_{\kappa} \cdot b_{\kappa} & =\frac{1}{3} \lambda_{\kappa}^{2}\left(\sum_{R_{\kappa}} x_{R_{\kappa}} C_{R_{\kappa}}-C_{\mathrm{Adj}_{\kappa}}\right), \\
b_{\kappa} \cdot b_{\mu} & =\lambda_{\kappa} \lambda_{\mu} \sum_{R_{\kappa}, R_{\mu}} x_{R_{\kappa}, R_{\mu}} A_{R_{\kappa}} A_{R_{\mu}}, \quad \kappa \neq \mu .
\end{aligned}
$$




\begin{tabular}{|cccccccccc|}
\hline & $\mathrm{A}_{n}$ & $\mathrm{~B}_{n}$ & $\mathrm{C}_{n}$ & $\mathrm{D}_{n}$ & $\mathrm{E}_{6}$ & $\mathrm{E}_{7}$ & $\mathrm{E}_{8}$ & $\mathrm{~F}_{4}$ & $\mathrm{G}_{2}$ \\
\hline$\lambda$ & 1 & 2 & 1 & 2 & 6 & 12 & 60 & 6 & 2 \\
\hline
\end{tabular}

Table 1. Normalization factors for the simple Lie groups.

The anomaly coefficients $a$ and $b_{\kappa}$ are $\mathrm{SO}(1, T)$ vectors in the string charge lattice $\Gamma$ of the $6 \mathrm{D}$ theory, and the notation $x \cdot y$ denotes the (integer-valued) $\mathrm{SO}(1, T)$-invariant product $\Omega_{\alpha \beta} x^{\alpha} y^{\beta}$ on $\Gamma$, where $\Omega$ is an invariant symmetric bilinear form in $\operatorname{SO}(1, T)$ associated with the Dirac pairing between string charges. Note that the indices $\kappa, \mu$ in eq. (2.9f) must be distinct. For each representation $R$ of a given gauge group factor, the group theory coefficients $A_{R}, B_{R}$, and $C_{R}$ are defined by

$$
\operatorname{tr}_{R} F^{2}=A_{R} \operatorname{tr} F^{2}, \quad \operatorname{tr}_{R} F^{4}=B_{R} \operatorname{tr} F^{4}+C_{R}\left(\operatorname{tr} F^{2}\right)^{2} .
$$

These group theory coefficients can be computed by hand for any given gauge group factor $\mathrm{SU}(N)$ in a manner discussed in [3] and [17], among other sources, and can also be determined systematically [18]. Note that for gauge groups without a quartic Casimir, like $\mathrm{SU}(2), \mathrm{SU}(3)$, and the exceptional groups, there is no coefficient $B_{R}$ and the anomaly equation $(2.9 \mathrm{~d})$ does not constrain the theory.

The $\lambda_{\kappa}$ are normalization factors associated with the simple nonabelian Lie groups (given by $\lambda_{\kappa}=2 c_{\kappa}^{\vee} / A_{\operatorname{Adj}_{\kappa}}$, where $c^{\vee}$ is the dual Coxeter number), given in table 1 .

The terms in the anomaly polynomial with abelian field strength factors yield the U(1) and mixed abelian-nonabelian anomaly equations, given by [19-21]

$$
\begin{aligned}
a \cdot b_{i j} & =-\frac{1}{6} \sum_{q_{i}, q_{j}} x_{q_{i}, q_{j}} q_{i} q_{j}, \\
0 & =\sum_{R_{\kappa}, q_{i}} x_{R_{\kappa}, q_{i}} E_{R_{\kappa}} q_{i}, \\
b_{\kappa} \cdot b_{i j} & =\lambda_{\kappa} \sum_{R_{\kappa}, q_{i}, q_{j}} x_{R_{\kappa}, q_{i}, q_{j}} A_{R_{\kappa}} q_{i} q_{j}, \\
b_{i j} \cdot b_{k \ell}+b_{i k} \cdot b_{j \ell}+b_{i \ell} \cdot b_{j k} & =\sum_{q_{i}, q_{j}, q_{k}, q_{\ell}} x_{q_{i}, q_{j}, q_{k}, q_{\ell}} q_{i} q_{j} q_{k} q_{\ell} .
\end{aligned}
$$

The $b_{i j}$ are $\mathrm{SO}(1, T)$ vectors in $\Gamma$, where, under the additional mild assumption that the $6 \mathrm{D}$ supergravity theory can be compactified on any spin manifold with any smooth gauge field configuration, we have the additional condition $b_{i i} \in 2 \Gamma[22]$, and the fourth group theory coefficient $E$ is defined by

$$
\operatorname{tr}_{R} F^{3}=E_{R} \operatorname{tr} F^{3}
$$

Note that the indices in eq. (2.11) need not be distinct.

We refer to eqs. (2.9) and (2.11) collectively as the anomaly cancellation (AC) equations.

In addition to the local anomalies, there are also global gauge anomalies that must cancel. These occur for gauge factors $\mathrm{SU}(2), \mathrm{SU}(3)$, and $\mathrm{G}_{2}$ [22-25]. In particular, for 
$\mathrm{SU}(2)$ and $\mathrm{SU}(3)$ with only fundamental and adjoint hypermultiplets, the conditions for the global anomalies to cancel are

$$
\begin{aligned}
\mathrm{SU}(2): & x \square+4 x_{\square} & =4(\bmod 6), \\
\mathrm{SU}(3): & x_{\square} & =0(\bmod 6) .
\end{aligned}
$$

The condition $b_{\kappa} \in \Gamma$ ensures that the cancellation of these global anomalies follows from the cancellation of the local gauge anomalies.

\section{Generic matter}

In this section we describe generic matter in $6 \mathrm{D}$ supergravity from several perspectives. We begin with gauge groups containing only up to three $\mathrm{U}(1)$ factors and only nonabelian $\mathrm{SU}(N)$ factors, and discuss at the end of the section how more $\mathrm{U}(1)$ factors and other nonabelian factors can be analyzed in a similar fashion. Note that the analysis in this section is based only on the structure of $6 \mathrm{D}$ supergravity theories and is independent of F-theory or any other UV completion.

\subsection{Generic matter representations for $G=\mathrm{SU}\left(N_{1}\right) \times \cdots \times \mathrm{SU}\left(N_{r}\right) \times \mathrm{U}(1)^{s}$, $s \leq 3$}

We begin by tabulating in table 2 the set of fields that we identify as living in generic matter representations for models of the form $G=\mathrm{SU}\left(N_{1}\right) \times \cdots \times \mathrm{SU}\left(N_{r}\right) \times \mathrm{U}(1)^{s}$, for $s \leq 3$. In the following parts of this section, we describe in detail the sense in which this set of representations can be considered "generic" from the points of view of moduli space dimension and anomaly cancellation. In each case we identify a canonical subset of the fields that matches the number of anomaly cancellation conditions. In all cases with no more than three abelian gauge factors, when there is a solution to the anomaly equations containing only the canonical set of generic matter fields, the dimensionality of the associated branch of the $6 \mathrm{D}$ supergravity theory should be greater than (or equal to) that of any other branch with the same discrete structure of tensor fields, gauge groups, anomaly coefficients, and string charge lattice. As discussed further below, when there are multiple abelian or nonabelian factors, the canonical set indicated in the table is not uniquely determined; for multiple abelian factors, different subsets of the full set of generic matter fields can be realized in models with different signs of anomaly coefficients. The generic matter fields in table 2 arise with non-negative multiplicities in solutions to the anomaly equations with small values of the anomaly coefficients; for nonabelian groups, the corresponding dimension of the moduli space branch for a given gauge theory is larger than for models with larger choices of anomaly coefficients. We illustrate the simplest examples of generic matter in section 3.2, and discuss in section 3.5 some aspects of how the definition of generic matter based on the dimension of moduli space branches relates to the choice of anomaly coefficients.

There is extra freedom in the set of generic matter fields when multiple gauge factors are involved, associated with the distinction between representations and their conjugates. This kind of ambiguity in generic matter arises in particular in distinguishing the $(\square, \square)$ 


\begin{tabular}{|c|c|c|}
\hline Canonical Representations & Number & Other Generic Representations \\
\hline $\mathbf{1}_{0}$ & 1 & \\
\hline$\square_{0}$ & $r$ & \\
\hline- & $r_{\geq 4}$ & \\
\hline $\operatorname{Adj}_{0}$ & $r$ & \\
\hline$(\square, \bar{\square})_{0}$ & $\left(\begin{array}{l}r \\
2\end{array}\right)$ & $(\square, \square)_{0}$ \\
\hline$\square_{1}$ & rs & \\
\hline$\square_{-1}$ & $\left(r_{3}+r_{\geq 4}\right) s$ & \\
\hline$\square_{(1,1)}$ & $r\left(\begin{array}{c}s \\
2\end{array}\right)$ & \\
\hline $\mathbf{1}_{1}$ & $s$ & \\
\hline $\mathbf{1}_{2}$ & $s$ & \\
\hline $\mathbf{1}_{(1,1)}$ & $\left(\begin{array}{l}s \\
2\end{array}\right)$ & \\
\hline $\mathbf{1}_{(1,-1)}$ & $\left(\begin{array}{l}s \\
2\end{array}\right)$ & \\
\hline $\mathbf{1}_{(2,-1)}$ & $2\left(\begin{array}{l}s \\
2\end{array}\right)$ & $\mathbf{1}_{(2,1)}$ \\
\hline $\mathbf{1}_{(1,1,-1)}$ & $3\left(\begin{array}{l}s \\
3\end{array}\right)$ & $\mathbf{1}_{(1,1,1)}$ \\
\hline
\end{tabular}

Table 2. Generic matter representations for gauge groups $G=\mathrm{SU}\left(N_{1}\right) \times \cdots \times \mathrm{SU}\left(N_{r}\right) \times \mathrm{U}(1)^{s}$ with $s \leq 3$, along with the number of distinct (canonical) representations of each type. Here, $r_{2}$, $r_{3}$, and $r_{\geq 4}$ are the number of $\mathrm{SU}(N)$ factors in $G$ with $N=2, N=3$, and $N \geq 4$, respectively, with $r=r_{2}+r_{3}+r_{\geq 4}$. Note that for some fields charged under multiple gauge factors, the fields in the first column are canonical choices and there are other "locally equivalent" generic matter representations, as discussed in section 3.4; such further generic matter representations are listed in the third column.

and $(\square, \square)$ representations of $\mathrm{SU}(N) \times \mathrm{SU}(M)$, as well as the $(2,1)$ vs. $(2,-1)$ representations of $\mathrm{U}(1)^{2}$ and the $(1,1,-1)$ vs. $(1,1,1)$ representations of $\mathrm{U}(1)^{3}$. In the first of these cases, the matter fields are locally indistinguishable (since they are equivalent under conjugating one of the gauge factors and they contribute equally to anomalies) and the branches of the moduli space with these alternate charges have equal dimension to the generic branch listed in table 2 . In the $\mathrm{U}(1)^{2}$ and $\mathrm{U}(1)^{3}$ cases, as discussed in sections 3.6 and 3.7, the situation is more subtle; different choices of signs of anomaly coefficients give rise to different combinations of generic matter fields that lie on branches of equal dimension.

We use in table 2 and in the rest of the paper a compressed notation for sets of representations, in which $\mathrm{SU}(N)$ representations are denoted with Young diagrams, $\mathrm{U}(1)$ charges are denoted with subscripts (except in some cases where there are no nonabelian factors), and only nontrivial representations are listed. In this way, we refer to a set 
of representations of a general given form. For example, the notation $\square(1,-1)$ actually designates the set

$$
\square_{(1,-1)}=\left\{(\mathbf{1}, \ldots, \mathbf{1}, \square, \mathbf{1}, \ldots, \mathbf{1})_{(0, \ldots, 0,1,0, \ldots, 0,-1,0, \ldots, 0)}\right\},
$$

while $(\square, \bar{\square})_{0}$ designates the set

$$
(\square, \bar{\square})_{0}=\left\{(1, \ldots, 1, \square, \mathbf{1}, \ldots, \mathbf{1}, \bar{\square}, \mathbf{1}, \ldots, 1)_{(0, \ldots, 0)}\right\} .
$$

These sets contain all representations with the nontrivial charges listed in any factor and in any permutation. Recall that a hypermultiplet we refer to as being in representation $R$ is in fact in the representation $R \oplus \bar{R}$, and so a negation of all $\mathrm{U}(1)$ charges and conjugation of all nonabelian reps does not result in a new representation.

\subsection{Examples: generic U(1) and SU(2) matter}

To illustrate the basic ideas we begin with a simple example: generic matter when the gauge group is just an abelian $\mathrm{U}(1)$ group. In this case, the anomaly equations are very simple, and read

$$
\begin{aligned}
-6 a \cdot \tilde{b} & =\sum_{q>0} x_{q} q^{2}, \\
3 \tilde{b} \cdot \tilde{b} & =\sum_{q>0} x_{q} q^{4} .
\end{aligned}
$$

Here, $\tilde{b}:=b_{11}$ is the single anomaly coefficient for the $\mathrm{U}(1)$ gauge factor, and $x_{q}$ is the number of fields of charge $q$.

In this case, the generic matter charges from table 2 are $q=1,2$. There are two independent anomaly equations constraining the charged matter, so for any anomaly coefficients $a, \tilde{b}$ there is a unique solution to the pair of equations

$$
\begin{aligned}
-6 a \cdot \tilde{b} & =x_{1}+4 x_{2}, \\
3 \tilde{b} \cdot \tilde{b} & =x_{1}+16 x_{2} .
\end{aligned}
$$

We can easily show explicitly that whenever there is a solution to these equations with non-negative charge multiplicities $x_{1}, x_{2}$, any other solution to the anomaly equations that contains higher charges will have fewer uncharged scalar degrees of freedom, indicating that the branches of supergravity moduli space with higher, "exotic," U(1) charges will have lower dimensionality. This can be seen by noting that the contribution to the anomaly equations from a single field of charge $q$ is equivalent to that of a linear combination of $y_{1}, y_{2}$ fields of charge 1,2 , respectively, determined by the equations

$$
\begin{aligned}
& q^{2}=y_{1}+4 y_{2}, \\
& q^{4}=y_{1}+16 y_{2} .
\end{aligned}
$$

The solution to these equations is $y_{1}=-q^{2}\left(q^{2}-4\right) / 3, y_{2}=q^{2}\left(q^{2}-1\right) / 12$. Because the total number of hypermultiplets is fixed by the gravitational anomaly condition (2.9a), this 
means that the following combinations of fields are equivalent under anomalies:

$$
(q)+\frac{q^{2}\left(q^{2}-4\right)}{3} \times(1) \longleftrightarrow \frac{q^{2}\left(q^{2}-1\right)}{12} \times(2)+\left(\frac{q^{4}}{4}-\frac{5 q^{2}}{4}+1\right) \times(0) .
$$

The number of uncharged scalars on the right-hand side is always positive for $q>2$, so starting with the generic matter solution with only charges $q=1,2$ and then "exchanging" these charges for any larger charge necessarily reduces the number of uncharged scalars. Since any solution to the anomaly equations can be found by a finite combination of such exchanges, this proves that the dimension of the moduli space on the branch with generic $\mathrm{U}(1)$ matter is larger than that of any branch with larger charges, when a solution with generic matter exists. Furthermore, as we discuss in more detail in section 3.5, when the anomaly coefficient $\tilde{b}$ is relatively small (subject to the condition that there is a solution of the anomaly equations with any set of charges), there is always a solution of eq. (3.4) with non-negative multiplicities $x_{1}, x_{2}$. Note that in this analysis, the equivalence (3.6) is simply used as a formal way of relating different solutions to the anomaly equations. In many cases, however, we expect that there are "matter transitions" in which these changes in spectrum can be realized physically without changing the gauge group [26]. We discuss "anomaly equivalences" like eq. (3.6) further in section 3.4.

The same kind of analysis can be carried out if the gauge group is SU(2). Again, there are only two anomaly equations constraining the charged matter since $\mathrm{SU}(2)$ has no quartic Casimir; these are

$$
\begin{aligned}
-6 a \cdot b & =x \square+4(x \square-1), \\
3 b \cdot b & =\frac{1}{2} x \square+8(x \square-1),
\end{aligned}
$$

with $b:=b_{\mathrm{SU}(2)}$. An arbitrary rep $\underbrace{\square \cdots \square}_{k}$ of $\mathrm{SU}(2)$ has $A_{R}=\left(\begin{array}{c}k+2 \\ 3\end{array}\right)$ and $C_{R}=\left(\begin{array}{c}k+2 \\ 3\end{array}\right) \frac{3 k(k+2)-4}{10}$. Expressing these as a linear combination of $A_{\square}=1, C_{\square}=\frac{1}{2}$ and $A_{\square}=4, C_{\square}=8$ leads to an anomaly equivalence

$$
\begin{aligned}
\underbrace{\square \cdots \square}_{k} & +\frac{(k+4)(k+2)(k+1) k(k-2)}{30} \times \square \\
& \longleftrightarrow\left(\begin{array}{c}
k+3 \\
5
\end{array}\right) \times \square \square+\frac{(k+4)(k+3)(k+1)(k-1)(k-2)}{24} \times \mathbf{1},
\end{aligned}
$$

where the number of uncharged scalars on the right-hand side is again always positive for $k>2$. So again, the dimension of the branch of moduli space with only fundamental and adjoint representations is larger than the branches containing any other combination of matter fields for a pure $\mathrm{SU}(2)$ theory. And again, as discussed in section 3.5, when $b$ is small, there is always a good solution of the anomaly equations with only fundamental and adjoint representations.

A similar analysis holds in an $\mathrm{SU}(2) \times \mathrm{U}(1)$ theory, where we expect that generic matter will only involve matter charged under the fundamental or adjoint of $\mathrm{SU}(2)$ that is neutral 
under the U(1) $\left(\square_{0}, \square_{0}\right)$, matter that is neutral under $\mathrm{SU}(2)$ and has charge $q=1$ or 2 under the $\mathrm{U}(1)$ factor $\left(\mathbf{1}_{1}, \mathbf{1}_{2}\right)$, and matter that lives in the fundamental representation of $\mathrm{SU}(2)$ and has charge $q=1$ under the $\mathrm{U}(1)\left(\square_{1}\right)$. In this case, there are five independent anomaly equations constraining the charged matter (the four considered above and one equation of the form (2.11c)), and correspondingly there are five charged generic matter fields. A similar calculation to those above shows that an exchange that increases the number of fields in some other representation $\underbrace{\square}_{k} \cdot \square_{q}$ by one decreases the number of uncharged scalars by

$$
\frac{(k+4)(k+3)(k+1)(k-1)(k-2)}{24}+\left(\frac{q^{4}}{4}-\frac{5 q^{2}}{4}\right)+\frac{k(k+1)(k+2) q^{2}}{6},
$$

which is positive for $k>2$, for $q>2$, for $k=2$ and $q>0$, or for $k=1$ and $q>1$.

All these cases provide simple examples of situations where we can explicitly show that the branch of moduli space with only generic matter types has greater dimension than any branch with other "exotic" matter types, and that these generic matter types can appear when the group theory anomaly coefficients $b$ are not too large. In the subsequent parts of this section, we explore the various perspectives on generic matter in more detail.

\subsection{Anomaly constraints and generic matter content}

Generalizing the examples just discussed, given a fixed gauge group $G$, and fixing the discrete parameters $T$ and $a, b_{\kappa}, b_{i j} \in \Gamma$, if we consider matter charged under a number of distinct representations equal to the number of nontrivial $\mathrm{AC}$ equations, then we can generally find a unique solution for the multiplicity $x_{R}$ of each representation $R$. In this sense, any set of anomaly-inequivalent generic matter charges realized on a maximal-dimensional moduli space branch should have a cardinality equal to the number of nontrivial AC equations. We now show that this is true for the canonical subsets of the generic matter charges listed in table 2.

For $\mathrm{SU}(N)$, we have $B_{R}=0$ for $N<4$ and $E_{R}=0$ for $N<3$. Suppose that

$$
G=\mathrm{SU}\left(N_{1}\right) \times \cdots \times \mathrm{SU}\left(N_{r}\right) \times \mathrm{U}(1)^{s}
$$

with $s \leq 3$, and let $r_{2}, r_{3}, r_{\geq 4}$ be the number of $\mathrm{SU}(N)$ factors in $G$ with $N=2, N=3$, and $N \geq 4$, respectively, with $r=r_{2}+r_{3}+r_{\geq 4}$. Examining the AC equations in the order presented (noting that eq. (2.9b) simply relates $a$ and $T$, and so does not contribute), we see then that the number of nontrivial $\mathrm{AC}$ equations is

$$
n_{\mathrm{AC}}\left(r_{2}, r_{3}, r_{\geq 4}, s\right)=1+r+r_{\geq 4}+r+\left(\begin{array}{l}
r \\
2
\end{array}\right)+\left(\left(\begin{array}{l}
s \\
2
\end{array}\right)\right)+\left(r_{3}+r_{\geq 4}\right) s+r\left(\left(\begin{array}{l}
s \\
2
\end{array}\right)\right)+\left(\left(\begin{array}{l}
s \\
4
\end{array}\right)\right),
$$

where

$$
\left(\left(\begin{array}{l}
n \\
k
\end{array}\right)\right)=\left(\begin{array}{c}
n+k-1 \\
k
\end{array}\right)
$$

gives the number of multisets of length $k$ on $n$ symbols. Note that the term $\left.\left(\begin{array}{l}s \\ 4\end{array}\right)\right)$ coming from eq. (2.11d) includes $\left(\begin{array}{l}s \\ 4\end{array}\right)=0$, because $s \leq 3$. 
As desired, the counting in $n(r, s)$ exactly matches the total number of representations in the canonical sets of generic matter fields in table 2 , noting that

$$
\left(\left(\begin{array}{l}
n \\
k
\end{array}\right)\right)=\sum_{j=1}^{k}\left(\begin{array}{l}
k-1 \\
j-1
\end{array}\right)\left(\begin{array}{l}
n \\
j
\end{array}\right) .
$$

\subsection{Anomaly-equivalent exchanges and ambiguities in generic matter}

As we have seen in some simple examples in section 3.2, there are many situations in which distinct combinations of matter representations will yield solutions to the AC equations 2.9 and (2.11) with the same anomaly coefficients on the left-hand side. For example, consider the $k=3$ case of eq. (3.8). In a $G=\mathrm{SU}(2)$ theory, the fundamental representation has $\left(A_{\square}, C_{\square}\right)=(1,1 / 2)$, the adjoint has $\left(A_{\square}, C_{\square}\right)=(4,8)$, and the triple-symmetric has $\left(A_{\square \square \square}, C_{\square \square \square}\right)=(10,41)$. Because these factors appear in the $\mathrm{AC}$ equations in sums over all hypermultiplets, we see that the exchange

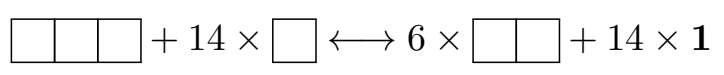

relates distinct solutions to the $\mathrm{AC}$ equations with the same $b_{\mathrm{SU}(2)}$. The singlet representations on the right side are necessary to balance the dimensions and satisfy the gravitational anomaly condition (2.9a). Two solutions to the AC equations related in this way are referred to as anomaly-equivalent. As mentioned earlier, in many cases there are paths in the supergravity moduli space that connect anomaly-equivalent matter spectra through matter transitions [26], though this need not always be the case.

In table 2, we have chosen a canonical set of generic matter representations with a cardinality that matches the number of AC equations. Thus, every spectrum that is allowed by anomaly cancellation will be anomaly-equivalent to some spectrum containing only the matter representations listed in this table (with the caveat that the resulting spectrum may have negative multiplicities for some representations). In many cases, exchanges will change the number of uncharged scalar moduli fields. As seen in eq. (3.14), increasing the number of triple-symmetric representations in an $\mathrm{SU}(2)$ theory requires reducing the number of moduli. In this sense, the triple-symmetric representation is non-generic (or "exotic"). More generally, from eq. (3.8), we see that the fundamental and adjoint representations of $\mathrm{SU}(2)$ are generic in the sense that any other anomaly-equivalent combination of representations will involve a smaller number of uncharged scalar moduli. We prove that the representations in table 2 are generic in the sense that exchanges that result in models containing only these representations can never decrease (but may increase) the number of uncharged scalars in appendix A.

It is important to note, however, that as the size of the gauge group increases, the set of matter representations that are generic in the sense of maximizing the dimension of the moduli space branch also increases, and can exceed the number of $\mathrm{AC}$ equations. In particular, when there are multiple gauge factors, there can be different anomaly-equivalent combinations of matter fields that have the same number of uncharged scalar moduli. In the simplest cases, there are certain combinations of matter representations under the factors of the gauge group that are "locally" indistinguishable in the sense that they can 
be related by conjugating one of the factors in the gauge group in a way that does not affect the anomaly conditions. For example, given a gauge group $\mathrm{SU}(N) \times \mathrm{SU}(M)$, the matter representations $(\square, \square)$ and $(\square, \square)$ are precisely equivalent under anomalies, since $A_{R}, B_{R}, C_{R}$ are unchanged under conjugation of the representation $R$. In fact, by choosing the conjugate realization of the gauge group $\mathrm{SU}(M)$, we can globally replace all matter in every representation $R$ with matter in the representation $\bar{R}$, so theories in which the complete spectrum is related by $R \leftrightarrow \bar{R}$ are globally equivalent. This leads to some ambiguity in the choice of generic matter for product gauge groups. One way to interpret this ambiguity is that, as we have done in table 2, we can choose a canonical set of generic matter representations whose cardinality matches the number of AC equations, and then there are other anomaly-free spectra in which these canonical choices can be freely exchanged for other generic matter representations that are anomaly-equivalent but not in the canonical set. Another way of framing this is simply to observe that the number of generic matter fields is larger than the number of AC equations, so that with sufficiently large product groups the $\mathrm{AC}$ equations do not uniquely determine the generic matter content of the theory. The situation is slightly more complicated for models with multiple $\mathrm{U}(1)$ factors, where for different choices of anomaly coefficients, different subsets of the set of generic matter fields are realized, in each case having cardinality equal to the number of $\mathrm{AC}$ equations; in these cases the different matter fields are similarly locally related by conjugation but contribute differently to the anomaly conditions. This is discussed in more detail for theories with two or three $\mathrm{U}(1)$ factors in sections 3.6 and 3.7. As we discuss in section 3.8, with four or more $\mathrm{U}(1)$ factors the number of generic matter fields with the same dimensionality of the moduli space branch increases still further.

In general, we will sometimes use the term "generic model" to describe a model living on a maximal-dimensional moduli space branch; except for the ambiguity between fundamental-fundamental and fundamental-antifundamental representations of $\mathrm{SU}(N) \times$ $\mathrm{SU}(M)$ product groups, and similar ambiguities discussed in section 3.8 that arise in the presence of four or more U(1) factors, we expect that such generic models will always have a number of anomaly-inequivalent matter representations equal to the number of anomaly equations.

\subsection{Generic matter and anomaly coefficients}

One subtlety in using the dimension of moduli space branches to define generic matter is that for different anomaly coefficients, different combinations of matter fields may be possible. To unambiguously define the generic matter representations we are interested in, we want to further focus on those representations that arise when the anomaly coefficients $b$ associated with the gauge group are relatively small. One could motivate this additional component in the precise definition of generic matter by some general sense in which small anomalies are more generic than large ones. A more satisfactory justification for focusing on small $b$ may be the fact that, at least for nonabelian groups, the branches of moduli space for a given gauge group, among those arising from all possible compatible anomaly coefficients, are largest when the $b$ s are small. While we do not attempt to prove rigorously for all the various nonabelian gauge groups considered here that the highest-dimensional branches 
of moduli space considered across all possible anomaly coefficients are always associated with small anomaly coefficients, we do show this in some of the simplest examples and also confirm in many cases that the matter content defined as generic in table 2 is realized with non-negative multiplicities at small $b$. It is also the case that the models with small anomaly coefficients and generic matter can generally be easily constructed in F-theory, while the explicit construction of models with larger anomaly coefficients is generally more difficult or impossible. In this subsection, we address this additional condition in the context of the simplest cases considered in section 3.2.

To give a sense of how the small $b$ condition affects the anomaly equations, we begin with a somewhat qualitative discussion. For simplicity, we focus in this discussion on the abelian anomaly equations and charges, though similar arguments hold in the nonabelian part of the theory. The abelian anomaly equations (2.11a) that are linear in $b$ are quadratic in the charges associated with the representations on the r.h.s., while the abelian anomaly equations that are quadratic in $b$ are quartic in the charges. (The corresponding statement for $\mathrm{SU}(2)$, for example, is that the anomaly coefficients $A_{k}, C_{k}$ for the $k$-index symmetric representation scale as the third and fifth powers of $k$ respectively, as noted in section 3.2). Thus, as the charges of the matter representations increase, the ratio between the r.h.s. of the equations quadratic in $b$ and those linear in $b$ increases roughly as $q^{2}$. For fixed $a$, then, we expect that small $b$ will generally be associated with anomaly solutions with nonnegative multiplicities for small charges. As $b$ increases, the typical charges of the solutions will increase. Thus, the types of matter that we are identifying as generic here can also be understood as those with relatively small charges. The exotic matter representations are those with larger charges, which arise for larger values of $b$. Note that for many gauge groups there are simple solutions with minimal anomaly coefficients $b$ in which only the representations with the very smallest charges have nonzero multiplicities (for example, for a U(1) theory with $b=6$ the spectrum contains 108 fields of charge $q=1$ and no other charges); we are interested in more generic situations where $b$ is large enough so that the number of representations with nonzero multiplicities is large enough to match the number of $\mathrm{AC}$ equations.

\subsubsection{SU(2) generic matter and anomaly coefficients}

To illustrate these issues, perhaps the simplest example is the case of theories with a single $\mathrm{SU}(2)$ factor and no tensor multiplets $(T=0)$. In this case, the gravitational anomaly coefficient is $-a=3$, and the anomaly equations restricted to the generic fundamental and adjoint matter fields are

$$
\begin{aligned}
& 18 b=x \square+4(x \square \square-1), \\
& 6 b^{2}=x \square^{+16(x \square \square-1),}
\end{aligned}
$$

where $b:=b_{\mathrm{SU}(2)}$ is a non-negative integer giving the anomaly coefficient for the $\mathrm{SU}(2)$ factor. It is straightforward to solve these equations, giving

$$
\begin{gathered}
x_{\square}=2 b(12-b), \\
x_{\square}=\frac{(b-1)(b-2)}{2} .
\end{gathered}
$$


Thus, when $b \leq 12$ there is a good solution (i.e., a solution with non-negative multiplicities for these generic matter fields) of the anomaly equations restricted to these two fields. Furthermore, the number of charged matter fields is

$$
H_{\text {charged }}=2 x \square+3 x \square \square=\frac{6+87 b-5 b^{2}}{2} .
$$

This number is smallest for small $b$; computing the dimension of the branch of moduli space, which is given from eq. (2.9a) by $H_{\text {uncharged }}=276-H_{\text {charged }}$, for $b \leq 12$ gives

$$
H_{\text {uncharged }}(b=1,2, \ldots, 12)=232,196,165,139,118,102,91,85,84,88,97,111 .
$$

This dimension is clearly largest at small values of $b$. When $b=13$, there is no solution with generic matter; we naively would have $x_{\square}=-26$ and $x_{\square}=66$. This is anomalyequivalent through eq. (3.14) to a model with spectrum

$$
b=13: \quad\left(x_{\square}, x_{\square}, x_{\square \square}\right)=(2,54,2), \quad H_{\text {uncharged }}=102 .
$$

A similar analysis up to $b=24$ gives models with fields in these three representations, and $H_{\text {uncharged }} \leq 102$. At this point, further representations such as $\square \square \square \square$ would need to be included for a non-negative spectrum. Thus, we see that for the gauge group SU(2) and theories with no tensor multiplets, the largest-dimensional branches of moduli space are associated with small values of $b$ and are realized by the generic matter content described in table 2 .

Continuing to consider the case of gauge group $\mathrm{SU}(2)$ and $T=0$, it is interesting to note that for certain values of $b$ there are anomaly-free non-negative spectra with positive values only for $x_{\square}, x_{\square \square}$; for example, at $b=14$ we have the spectrum

$$
b=14: \quad\left(x_{\square}, x_{\square}, x_{\square \square}\right)=(0,54,4), \quad H_{\text {uncharged }}=98 .
$$

For this choice of anomaly coefficient, this spectrum maximizes the dimension of the branch of moduli space. Thus, if we did not consider the range of anomaly coefficients $b$ or make an absolute comparison between the dimensions of branches for different values of $b$, it might seem natural to define generic matter as depending on $b$, so that the representations $\square \square, \square=\mid$ would be "generic" matter for $b=14$. From this point of view, eq. (3.14) could be viewed as increasing the dimensionality of the moduli space branch by trading away all fundamentals to end up at the maximal-dimensional branch with only representations $\square, \square, \square \quad \square$. We choose to focus on the types of matter that arise at small $b$ for several reasons: first, this gives a more universal definition of generic matter; second, this definition matches with the branches of moduli space of largest dimension compared across anomaly coefficients for nonabelian groups such as $\mathrm{SU}(2)$; and third, this matches most naturally with the structures we find from F-theory, as described later in the paper. 


\subsubsection{U(1) generic matter and anomaly coefficients}

Now let us consider the case of generic matter for models with a gauge group U(1), again restricting to the case $T=0$, where $-a=3$ and $\tilde{b}:=b_{11}$ is a non-negative integer. In this case, there is an additional subtlety coming from the fact that for the smallest values of $\tilde{b}$ there are no solutions at all to the anomaly equations with non-negative spectra. In particular, we can see immediately by taking the difference of the equations (3.3) that

$$
3 \tilde{b}(\tilde{b}-6)=\sum_{q>1} x_{q} q^{2}\left(q^{2}-1\right)
$$

so there can only be a non-negative spectrum for any set of charges when $\tilde{b} \geq 6$. Thus, when we assert that generic matter representations should be associated with the matter fields arising in the moduli space branch of highest dimension when the anomaly coefficients are small, we must include the condition that the anomaly coefficients be at least big enough that there is a possible non-negative spectrum.

With this condition, it is straightforward to compute the $\mathrm{U}(1)$ spectrum with charges $q=1,2$ when $\tilde{b}$ is small but not less than 6 . The result is

$$
\begin{aligned}
& x_{1}=\tilde{b}(24-\tilde{b}), \\
& x_{2}=\frac{\tilde{b}(\tilde{b}-6)}{4} .
\end{aligned}
$$

This spectrum is simply that associated with Higgsing the $\mathrm{SU}(2)$ models in eq. (3.16), when there is at least one adjoint matter field (see [5] for further related analysis). From this, it is clear that there is an acceptable spectrum of the $\mathrm{U}(1)$ model with these charges whenever $\tilde{b}$ is even and $6 \leq \tilde{b} \leq 24$ (note that, from eq. (3.21), there cannot be any solutions to the anomaly equations when $\tilde{b}$ is odd).

Note that for the U(1) gauge group, the dimension of the branches of moduli space does not decrease with increasing $\tilde{b}$ in the same way that it does for $\mathrm{SU}(2)$ and other nonabelian gauge groups. This is related to the fact that for $\mathrm{U}(1)$ there are an infinite number of possible solutions of the anomaly equations with arbitrarily large charges [5], unlike the finite number of matter spectra allowed for a fixed nonabelian gauge group, which follows from the fact that the dimensions of nonabelian group representations increase so that only a finite set of distinct representations are allowed for a theory with a given nonabelian gauge group, from the gravitational anomaly condition (2.9a).

\subsubsection{Models with $T>0$}

In the examples we have considered thus far, we have focused on the case with no tensor multiplets $(T=0)$. The observation that the generic matter fields are realized with nonnegative multiplicities in solutions to the anomaly equations for small values of the anomaly coefficients $b$ also holds more generally, though the analysis is a bit more involved.

Part of the challenge in dealing with models at larger values of $T$ is that the constraints on the anomaly coefficient $a$ and the positivity cone in the string charge lattice are not as well understood from the low-energy point of view [4]. Here, we briefly consider the analysis 
for the gauge group $\mathrm{SU}(2)$ at $T=1$ using the simplest positivity cones that are known to come from F-theory; a further analysis for larger values of $T$ is included in appendix B.

When $T>0$, the string charge lattice is a unimodular lattice $\Gamma[27]$ and $-a$ is a characteristic vector in $\Gamma[22]$ that satisfies $a \cdot a=9-T$. The anomaly coefficients $b_{i i}, b_{\kappa}$ for each abelian and nonabelian factor lie in a positivity cone in $\Gamma$ (this provides the proper sign for the gauge kinetic terms). For $T=1$, the two simplest choices of inner products associated with $\Gamma$ and positivity cones that come from F-theory are

$$
(x, y) \cdot\left(x^{\prime}, y^{\prime}\right)=x y^{\prime}+y x^{\prime} ; \quad x, y \geq 0
$$

and

$$
(x, y) \cdot\left(x^{\prime}, y^{\prime}\right)=x x^{\prime}-y y^{\prime} ; \quad x, y+x \geq 0 .
$$

We consider these cases in turn. (The structure of these two unimodular lattices $\Gamma$ is described in more detail in section 4.3.1.)

In the first, "even" case (3.23), we can choose a basis where $-a=(2,2)$. The $\mathrm{SU}(2)$ anomaly equations for a gauge factor with anomaly coefficient $b=\left(b_{0}, b_{1}\right)$ are then

$$
\begin{array}{r}
-6 a \cdot b=12\left(b_{0}+b_{1}\right)=x \square+4(x \square-1), \\
6 b \cdot b=12 b_{0} b_{1}=x \square+16(x \square \square-1) .
\end{array}
$$

Solving for generic SU(2) matter, we have

$$
\begin{gathered}
x_{\square}=16\left(b_{0}+b_{1}\right)-4 b_{0} b_{1}, \\
x_{\square \square}=1+b_{0} b_{1}-b_{0}-b_{1} .
\end{gathered}
$$

As shown in figure 1 , these equations have non-negative solutions whenever $b_{0}, b_{1} \leq 8$, except at $b_{0}=0, b_{1}>0$ and $b_{0}>0, b_{1}=0$. However, there are no solutions at all to the AC conditions for choices of $b$ where $b_{i}=0, b_{1-i}>1$ : as discussed in appendix B, any valid model must have

$$
g=\frac{b \cdot(b+a)}{2}+1 \geq 0 .
$$

We see that when $b_{i}=0, g=1-b_{1-i}$, and so there are no valid solutions in these cases. Alternatively, we see that for solutions in terms of generic matter, $x \square \square=g$, so any choice of $b_{i}$ for which the generic matter solution has a negative multiplicity of adjoints has no good solution for any set of representations. Thus, there are valid generic matter models for all $b_{0}, b_{1} \leq 8$ in the positivity cone for which there are any valid solutions.

We can use a similar approach for the "odd" case (3.24). Here, we can choose a basis where $-a=(3,-1)$. The $\mathrm{SU}(2)$ anomaly equations then become

$$
\begin{array}{r}
-6 a \cdot b=6\left(3 b_{0}+b_{1}\right)=x \square+4(x \square \square-1), \\
6 b \cdot b=6\left(b_{0}^{2}-b_{1}^{2}\right)=x \square+16(x \square \square-1),
\end{array}
$$

yielding

$$
\begin{gathered}
x_{\square}=24 b_{0}+8 b_{1}-2 b_{0}^{2}+2 b_{1}^{2}, \\
x_{\square \square}=\frac{1}{2}\left(b_{0}^{2}-b_{1}^{2}-3 b_{0}-b_{1}+2\right) .
\end{gathered}
$$




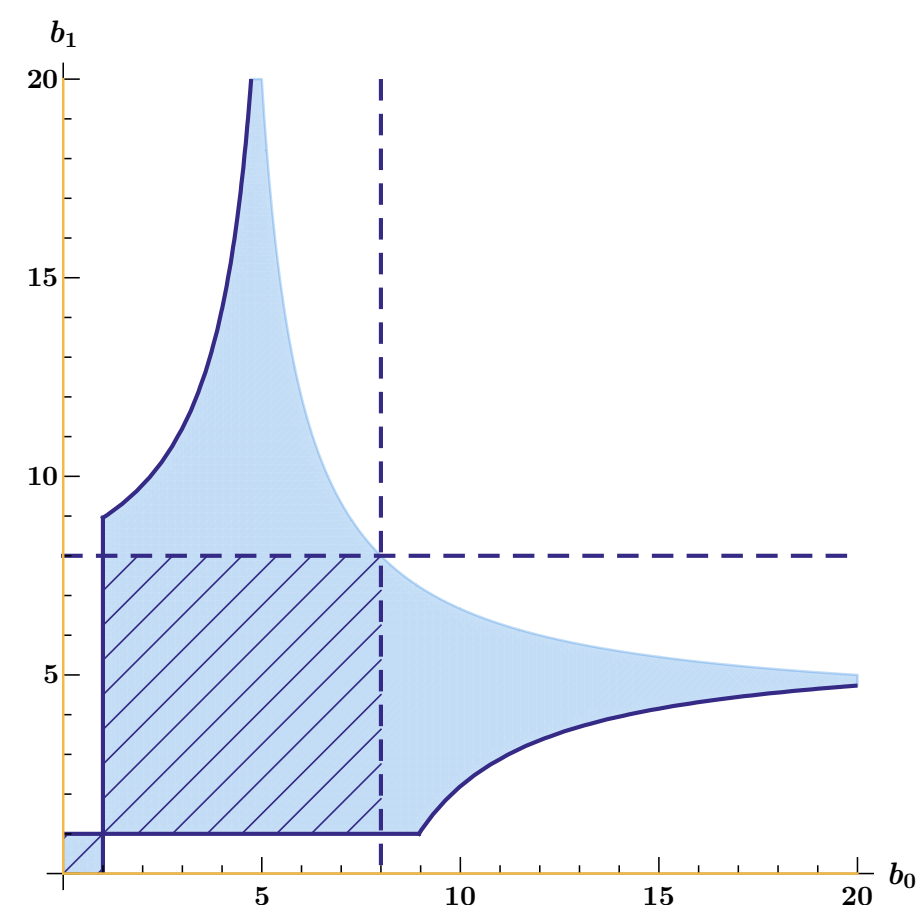

Figure 1. Plot of the $\left(b_{0}, b_{1}\right)$-plane for an $\mathrm{SU}(2)$ model with $T=1$, even string charge lattice, and positivity cone $b_{0}, b_{1} \geq 0$. Choices of $b=\left(b_{0}, b_{1}\right)$ within the positivity cone (bounded by the yellow curves) for which the AC equations have non-negative solutions with only generic matter are shaded in solid blue. The solid curves denote $g=0\left(b_{0}, b_{1}=1\right)$ and the gravitational bound (2.9a) on generic matter, which becomes a bound on $b_{0}, b_{1}$ using eq. (3.26). The dashed curves are $b_{0}, b_{1}=8$. The solid and dashed curves bound the hashed region where $g \geq 0$ (a constraint necessary to have any good solutions to the $\mathrm{AC}$ conditions), the gravitational bound is satisfied, and $b_{0}, b_{1} \leq 8$ (the small $b$ constraint). The hashed region lies entirely within the solid shaded region, showing that all such choices of $b$ yield non-negative solutions of the AC equations with only generic matter. As described in section 4.3.3, the models within the hashed region have a simple F-theory construction using a Tate tuning, while the models in the shaded but not hashed region would require a more sophisticated F-theory Weierstrass model, which may or may not exist.

As above, a choice of $b$ that yields any good solution of the $\mathrm{AC}$ equations will have

$$
x_{\square}=g \geq 0
$$

for the solution with generic matter.

As shown in figure 2, the multiplicities (3.29) are positive for all choices of $b$ with $b_{0} \leq 12$ and $b_{0}+b_{1} \leq 8$ that satisfy $g \geq 0$. Thus, we see that generic matter is realized with non-negative multiplicities for appropriate small values of $b$ in the positivity cone on the odd lattice as well.

In appendix B, we use a different approach to demonstrate these results for higher $T>0$ in a way that does not rely on a choice of positivity cone. 


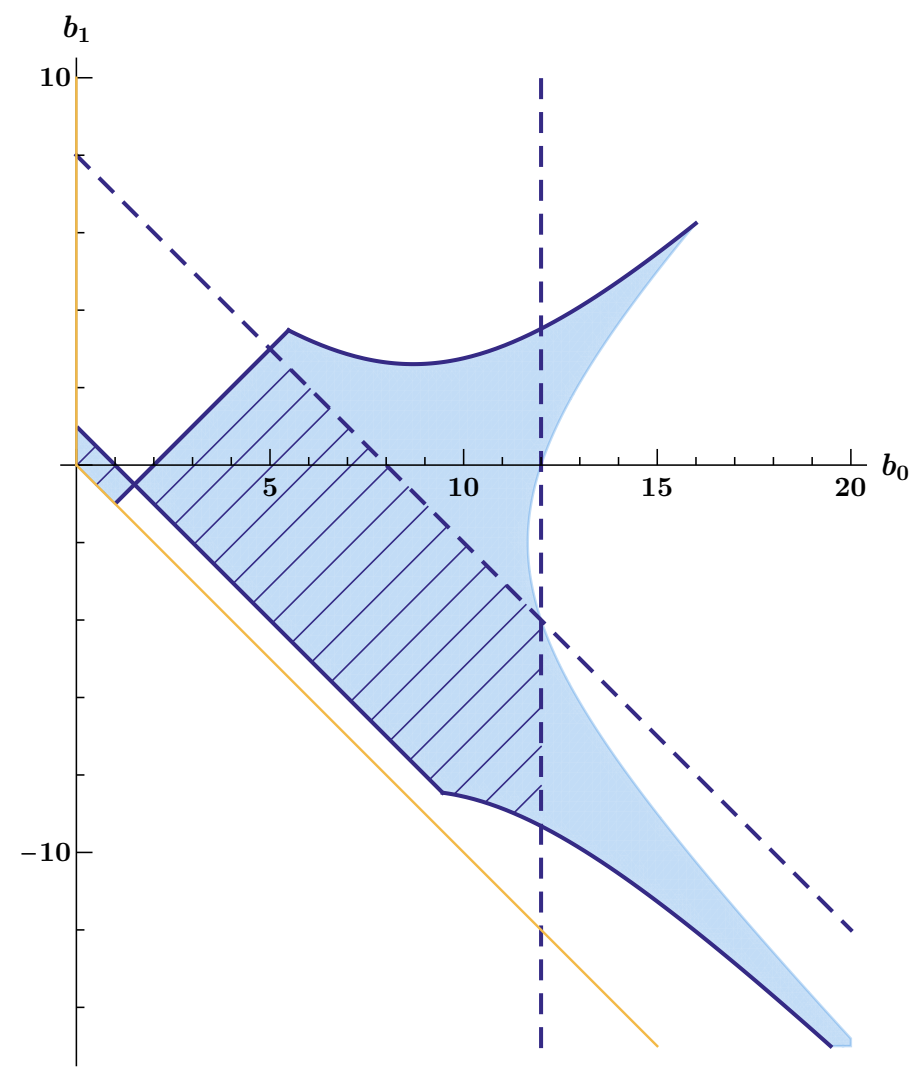

Figure 2. Plot of the $\left(b_{0}, b_{1}\right)$-plane for an $\mathrm{SU}(2)$ model with $T=1$, odd string charge lattice, and positivity cone $b_{0}, b_{1}+b_{0} \geq 0$. Choices of $b=\left(b_{0}, b_{1}\right)$ within the positivity cone (bounded by the yellow curves) for which the $\mathrm{AC}$ equations have non-negative solutions with only generic matter are shaded in solid blue. The solid curves denote $g=0\left(b_{0}+b_{1}=1, b_{0}-b_{1}=2\right)$ and the gravitational bound (2.9a) on generic matter, which becomes a bound on $b_{0}, b_{1}$ using eq. (3.29). The dashed curves are $b_{0}=12, b_{0}+b_{1}=8$. The solid and dashed curves bound the hashed region (again indicating the models with an F-theory Tate tuning) where $g \geq 0$ (a constraint necessary to have any good solutions to the $\mathrm{AC}$ conditions), the gravitational bound is satisfied, and $b_{0} \leq 12, b_{0}+b_{1} \leq 8$ (the small $b$ constraint). The hashed region lies entirely within the solid shaded region, showing that all such choices of $b$ yield non-negative solutions of the AC equations with only generic matter.

\subsection{Two abelian factors: $\mathrm{U}(1)^{2}$}

When the gauge group contains multiple abelian U(1) factors, the structure of generic matter becomes more complicated. In particular, the number of generic matter fields becomes larger than the number of anomaly cancellation equations, and different choices of anomaly coefficients give rise to different subsets of the set of generic matter fields. In the case of two $\mathrm{U}(1)$ factors, the fields

$$
(1, \pm 1), \quad(2, \pm 1), \quad(1, \pm 2)
$$

are all included in the set of generic matter fields. In any valid "generic" model (i.e., with a maximal-dimensional branch of moduli space for given relatively small anomaly coefficients), however, at most four of these six fields have nonzero multiplicities. This matches 
with the number expected from the anomaly cancellation conditions. The way in which these fields appear, however, is somewhat different than in the previously examined cases.

\subsubsection{Choices of generic matter spectra for $U(1) \times U(1)$ theories}

The fields in eq. (3.31) are related in pairs by conjugating one of the $U(1)$ factors, similar to the story with $(\square, \square)$ and $(\square, \square)$ matter fields for the group $\mathrm{SU}(N) \times \mathrm{SU}(M)$. When abelian U(1) factors are involved, however, even matter fields that are locally equivalent under a conjugation of a gauge factor can be anomaly-inequivalent. For example, with a gauge group of $\mathrm{SU}(N) \times \mathrm{U}(1)$, the representations $\square_{1}$ and $\square_{-1}$ are locally equivalent, in that one representation switches to the other under a conjugation of either gauge factor; however, these representations are actually distinguished by the anomaly equation (2.11b), and hence are not anomaly-equivalent and are separately listed as generic matter representations in the canonical set in table 2 that matches the number of AC equations. Similarly, for $\mathrm{U}(1)^{2}$ the representations $(1,1)$ and $(1,-1)$ are distinguished by the anomaly equations (2.11), as are the representations $(2, \pm 1)$, etc.

The abelian and mixed abelian-nonabelian anomaly equations (2.11) have the feature that they are invariant under a simultaneous change of signs

$$
\begin{aligned}
q_{i} & \rightarrow-q_{i}, \\
b_{i j} & \rightarrow-b_{i j}, \quad \forall j \neq i
\end{aligned}
$$

for any fixed choice of $i$. Thus, in a theory with $G=\mathrm{U}(1) \times \mathrm{U}(1)$, a valid spectrum of matter fields $x_{p, q}$ that solve the anomaly equations with a fixed anomaly coefficient $b_{12}$ is related to another solution with the anomaly coefficient $b_{12}^{\prime}=-b_{12}$, where the multiplicities of the matter fields in the sign-flipped solution are

$$
b_{12} \rightarrow b_{12}^{\prime}=-b_{12} \quad \Rightarrow \quad x_{p, q}^{\prime}=x_{p,-q} .
$$

While all six fields in eq. (3.31) can arise as generic matter fields, for any fixed choice of anomaly coefficients we expect from the number of $\mathrm{AC}$ equations that at most four of these fields will have positive multiplicities in a valid generic model with a maximal dimension of the moduli space branch. Indeed, this is the case. While generic $\mathrm{U}(1) \times \mathrm{U}(1)$ models contain fields of both sign choices $(1, \pm 1)$, the solutions with the largest number of uncharged scalars (at small $b$ ) have only one each taken from the pairs $(2, \pm 1)$ and $(1, \pm 2)$. In table 2, we have made a canonical choice of the fields with both signs negative, but the other three choices of sign combinations are also possible.

One way to understand this is from anomaly equivalences. From the anomaly equations, we can find the anomaly equivalence

$$
(2,1)+(2,-1)+6 \times(0,1)+8 \times(1,0) \longleftrightarrow 2 \times(2,0)+4 \times(1,1)+4 \times(1,-1)+6 \times(0,0) .
$$

Thus, we can always exchange a pair of charges $(2,1)+(2,-1)$ for other generic matter (assuming a sufficient number of fields of charges $(0,1)$ and $(1,0))$ and increase the number of uncharged scalars. A similar statement clearly holds for $(1,2)+(-1,2)$ matter. When 
we only have $(2,1)$ matter fields and no fields of charge $(2,-1)$, however, we cannot make further exchanges without decreasing the number of uncharged scalars. Thus, a model with generic matter is expected to have only one of the types of charge $(2,1)$ or $(2,-1)$, and only one of the types of charge $(1,2)$ or $(-1,2)$.

In fact, the sign choices of the allowed matter fields are determined directly by the structure of the anomaly coefficients. As described in more detail in appendix C, solving the eight relevant anomaly equations for the canonical choice of generic $\mathrm{U}(1) \times \mathrm{U}(1)$ matter content in table 2 gives

$$
\begin{aligned}
& x_{2,-1}=-b_{12} \cdot\left(a+\frac{1}{2} b_{11}\right), \\
& x_{-1,2}=-b_{12} \cdot\left(a+\frac{1}{2} b_{22}\right) .
\end{aligned}
$$

These multiplicities are non-negative when the r.h.s. of both equations is non-negative. This combination of signs for the r.h.s. of eq. (3.35) and the subset of generic matter fields containing $(2,-1),(-1,2)$ represents one consistent class of models associated with specific signs for the anomaly coefficients. Because there is no positivity constraint on the anomaly coefficient $b_{12}$, we can consider another closely related model, as discussed above, in which the sign of this coefficient is flipped; this corresponds to an equivalent model in which one of the $\mathrm{U}(1)$ factors is conjugated, giving a model with a spectrum related to the original spectrum through eq. (3.33). In particular, the resulting model has generic matter fields of types $(2,1)$ and $(1,2)$. Indeed, explicitly solving the eight relevant anomaly equations for the generic matter content with this choice of fields gives

$$
\begin{aligned}
& x_{2,1}=b_{12} \cdot\left(a+\frac{1}{2} b_{11}\right), \\
& x_{1,2}=b_{12} \cdot\left(a+\frac{1}{2} b_{22}\right) .
\end{aligned}
$$

When $T=0$, we can show that in fact these two types of matter spectra are the only possible combinations consistent with the anomaly constraints. From eq. (3.4) (noting that these equations will hold separately for each U(1) factor), we see that for each $U(1)$ factor we have

$$
x_{2}^{i}=\left(\frac{1}{2} b_{i i}\right) \cdot\left(a+\frac{1}{2} b_{i i}\right) \geq 0 .
$$

For theories with $T=0$, the anomaly coefficients $a, b_{i j}$ are integers, and $-a=3$, so eq. (3.4a) implies that $b_{i i} \geq 0$. This implies that for each $i, a+b_{i i} / 2 \geq 0$. As a result, the r.h.s. of eq. (3.35) always both have the same sign when $T=0$, as do the r.h.s. of eq. (3.36).

When $T>0$, however, the terms on the r.h.s. of the two equations (3.35) can have opposite signs. In this case, we get a mixed spectrum, with either charges $(2,1),(-1,2)$ or $(1,2),(2,-1)$. In these cases, the expressions for the multiplicities of the non-negative charges of these types are given by one equation from eq. (3.35) and one equation from eq. (3.36), as appropriate. We describe an explicit example of such a spectrum below. 


\subsubsection{Generic $\mathrm{U}(1) \times \mathrm{U}(1)$ matter from Higgsing nonabelian theories}

One illuminating perspective on these spectra can be understood from the point of view of Higgsing a nonabelian theory. As described in $[28]^{2}$, the canonical $\mathrm{U}(1) \times \mathrm{U}(1)$ generic matter types from table 2 can be realized when a theory with nonabelian gauge group $G_{(2)}=\mathrm{SU}(2) \times \mathrm{SU}(2) \times \mathrm{SU}(3)$ and generic matter is broken by Higgsing pairs of bifundamental $(\square, \mathbf{1}, \square)$ and $(\mathbf{1}, \square, \square)$ fields to give the $\mathrm{U}(1) \times \mathrm{U}(1)$ model. $^{3}$

In particular, we can embed the two $\mathrm{U}(1)$ factors into $G_{(2)}$ as

$$
\begin{gathered}
\mathrm{U}(1)_{1} \rightarrow\left(\begin{array}{cc}
1 & 0 \\
0 & -1
\end{array}\right)_{1}+\left(\begin{array}{ll}
0 & 0 \\
0 & 0
\end{array}\right)_{2}+\left(\begin{array}{ccc}
1 & 0 & 0 \\
0 & -1 & 0 \\
0 & 0 & 0
\end{array}\right)_{3}, \\
\mathrm{U}(1)_{2} \rightarrow\left(\begin{array}{ll}
0 & 0 \\
0 & 0
\end{array}\right)_{1}+\left(\begin{array}{cc}
1 & 0 \\
0 & -1
\end{array}\right)_{2}+\left(\begin{array}{ccc}
0 & 0 & 0 \\
0 & 1 & 0 \\
0 & 0 & -1
\end{array}\right)_{3} .
\end{gathered}
$$

Here, the subscript on each matrix indicates which factor of $G_{(2)}$ it acts in. With this embedding, we see that the generic matter types for $G_{(2)}$ descend to the canonical generic matter types for $\mathrm{U}(1) \times \mathrm{U}(1)$. For example, the adjoint of $\mathrm{SU}(3)$ contains fields that will give charges $(2,-1)$ and $(-1,2)$, as do the bifundamental fields $(\square, \mathbf{1}, \square)$ and $(\mathbf{1}, \square, \square)$.

If we denote the anomaly coefficients in the nonabelian $G_{(2)}$ model by $A=b_{\mathrm{SU}(2)_{1}}, B=$ $b_{\mathrm{SU}(2)_{2}}, C=b_{\mathrm{SU}(3)}$, then it is straightforward to deduce that

$$
\begin{aligned}
& b_{11}=2(A+C) \\
& b_{22}=2(B+C) \\
& b_{12}=-C .
\end{aligned}
$$

With the canonical choice of generic matter and the above embedding, we can then interpret the multiplicities (3.35) in terms of the original nonabelian model. In particular,

$$
x_{2,-1}=C \cdot(a+A+C)=C \cdot A+2 g_{C}-2,
$$

and a similar relation holds for $x_{-1,2}$, where $g_{C}=C \cdot(a+C) / 2+1$. This immediately matches with the analysis above: $C \cdot A$ is the number of $(\square, \mathbf{1}, \square)$ fields (which give one $(2,-1)$ field each), $g_{C}$ is the number of adjoints (which give two $(2,-1)$ fields each), and two bifundamental fields need to be used for the Higgsing.

From this point of view, many consistent generic $\mathrm{U}(1) \times \mathrm{U}(1)$ spectra can be realized by Higgsing a nonabelian model with gauge group $G_{(2)}$. The alternative spectra with $(2,1)$

\footnotetext{
${ }^{2}$ In the model in [28], the sign of $b_{12}$ is such that the spectrum contains $(2,1),(1,2)$ charged matter, but the embedding construction there is essentially equivalent to that used in the discussion here to realize our choice here of canonical $\mathrm{U}(1) \times \mathrm{U}(1)$ matter.

${ }^{3}$ Note that in this and the following sections on $\mathrm{U}(1)^{s}$ models for $s=3, s>3$, we choose to use fundamental-fundamental $\mathrm{SU}(N) \times \mathrm{SU}(M)$ matter as canonical rather than fundamental-antifundamental matter to simplify the structure of the Higgsing and embedding formulae; a different choice of signs for the embedding would match with the fundamental-antifundamental canonical matter choice in table 2 .
} 
and $(1,2)$ matter can be realized similarly by using eq. (3.32), which can be done explicitly by simply flipping the sign of one of the $\mathrm{U}(1)$ factors in the embedding (3.38) and changing the sign of $b_{12}$. Not all generic $\mathrm{U}(1) \times \mathrm{U}(1)$ models can be unHiggsed in this fashion, however. In some cases, there may not be enough uncharged scalar matter. Another more interesting class of cases arises when we have the mixed matter types such as $(2,1)$ and $(1,-2)$. We conclude this section with a simple example of such a case.

Consider the case of $T=1$ with the odd string lattice and positivity cone (3.24). We choose

$$
b_{11}=(6,0), \quad b_{22}=(8,-4), \quad b_{12}=(0,1)
$$

We see that in this case, we expect the spectrum

$$
\begin{gathered}
x_{2,-1}=-b_{12} \cdot\left(a+\frac{1}{2} b_{11}\right)=1, \\
x_{1,2}=b_{12} \cdot\left(a+\frac{1}{2} b_{22}\right)=3 .
\end{gathered}
$$

This cannot come from a Higgsed nonabelian model as described above, however. Choosing, for example, $C=b_{12}$ and $A=b_{11} / 2-C$, we see that the nonabelian model would have no SU(3) adjoint matter and only one $(\square, \mathbf{1}, \square)$ bifundamental field, so there would not be sufficient matter available for the Higgsing. Nonetheless, as we discuss further in section 4, it seems likely that this $\mathrm{U}(1) \times \mathrm{U}(1)$ spectrum can be realized in F-theory.

\subsubsection{Generic $\mathrm{U}(1) \times \mathrm{U}(1)$ matter at small $b, T=0$}

We believe that for all sufficiently small choices of $b$ that have good solutions to the AC conditions, a subset of generic matter representations with cardinality equal to the number of nontrivial AC conditions is realized with non-negative multiplicities. We prove that this is the case for $T=0$ models here, and briefly discuss the more general case in appendix C.

We have already seen in section 3.6.1 that for any choice of $b_{i j}$ that yields good solutions of the $\mathrm{AC}$ equations, at most one of the pairs $(2,-1),(-1,2)$ and $(2,1),(1,2)$ can have nonzero multiplicities in a generic model on a maximal-dimensional branch of moduli space. Thus, we only need to inspect the multiplicities for the remaining generic matter representations. The full set of equations determining these multiplicities is given in appendix C. For $T=0$, we find that for all $b_{i i}$ with $b_{11}+b_{22} \leq 24$ that yield good solutions of the AC equations, there exists a good solution with generic matter for one of the two choices of $(2, \pm 1),( \pm 1,2)$ for all $\left|b_{12}\right| \leq \min \left(b_{11}, b_{22}\right) / 2$. This result is shown in figure 3 .

\subsection{Three abelian factors: $\mathrm{U}(1)^{3}$}

Turning to $\mathrm{U}(1)^{3}$, in addition to the canonical fields in table 2, matter fields of the form $(1,1,1)$ are locally equivalent to those with charges $(1,1,-1)$, and can also play the role of generic matter fields. Similarly to the case of charges $(2,1)$ and $(2,-1)$, we find that there 


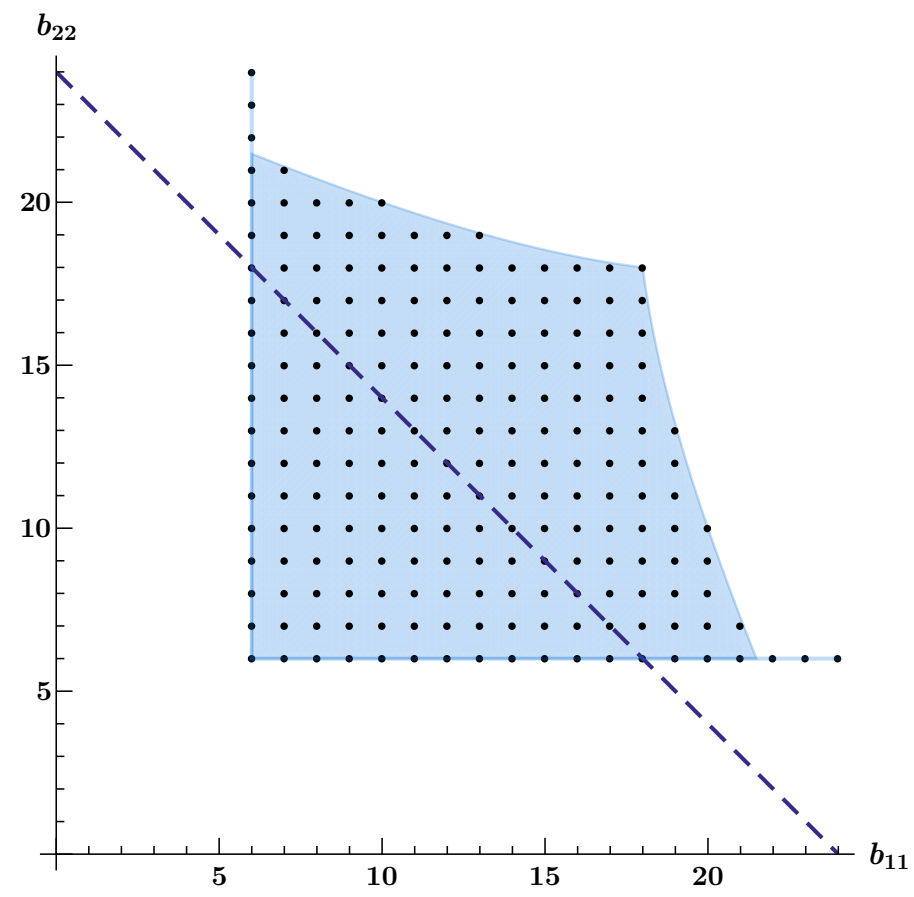

Figure 3. Plot of the $\left(b_{11}, b_{22}\right)$-plane for a $\mathrm{U}(1) \times \mathrm{U}(1)$ model with $T=0$. Choices of $b_{11}, b_{22}$ for which the AC equations have non-negative solutions with only generic matter (either with the $(2,-1),(-1,2)$ or the $(2,1),(1,2)$ pair of charge combinations) are shaded in solid blue. Integer choices of $b_{i i}$ for which there is such a solution with integer $b_{i j}$ are superimposed in black. Below the dashed curve $b_{11}+b_{22}=24$, all choices of $b_{i i}$ that yield any good solutions of the AC equations lie within the blue shaded region, and within this region, there is a good solution with one of the two above subsets of generic matter for all $\left|b_{12}\right| \leq \min \left(b_{11}, b_{22}\right) / 2$.

is an exchange

$$
\begin{aligned}
& (1,1,1)+(-1,1,1)+(1,-1,1)+(1,1,-1) \\
& +4 \times(1,0,0)+4 \times(0,1,0)+4 \times(0,0,1) \\
& \longleftrightarrow \\
& 2 \times(1,1,0)+2 \times(1,0,1)+2 \times(0,1,1)+2 \times(1,-1,0) \\
& +2 \times(1,0,-1)+2 \times(0,1,-1)+4 \times(0,0,0),
\end{aligned}
$$

so that, assuming there are a sufficient number of fields of charges $(1,0,0),(0,1,0)$, and $(0,0,1)$, we can always remove quartets of charges $(1,1,1)+(-1,1,1)+(1,-1,1)+(1,1,-1)$ and increase the number of uncharged scalars. Thus, we expect that models with generic matter and a maximal number of uncharged scalars (at small $b$ as usual) should only have a size three subset of these four charges present. Note that the number of canonical fields listed in the table has cardinality $3\left(\begin{array}{l}s \\ 3\end{array}\right)$, which matches the number of AC equations, while the number of fields of type $(1,1,1)$ is $\left(\begin{array}{c}s \\ 3\end{array}\right)$.

For each pair of $\mathrm{U}(1)$ charges, we can again use the analysis of the previous section to show that there are only one each of the pairs $(2, \pm 1,0),(1, \pm 2,0)$, etc. Furthermore, 
because the analysis for each pair of $\mathrm{U}(1)$ factors considered independent of the third charge is unchanged, we can read off which elements of each of the $(2, \pm 1,0)$ type pairs are realized directly from the signs of the terms $b_{i j} \cdot\left(a+b_{i i} / 2\right)$, analogous to eqs. (3.35) and (3.36), for each pair $i, j$.

While the structure of the minimal set of three of the four $(1, \pm 1, \pm 1)$ type fields (recall that $(1,-1,-1)$ is the same type of matter as $(-1,1,1))$ is correlated with the structure of the $(2, \pm 1,0)$ type fields, in some cases the minimal set of $(1, \pm 1, \pm 1)$ fields is not determined uniquely by the $(2, \pm 1,0)$ type fields or by the signs of the terms $b_{i j} \cdot\left(a+b_{i i} / 2\right)$. To say a little more about this, it is helpful to distinguish two classes of configurations. To illustrate the first class, let us start with a case where $-b_{i j} \cdot\left(a+b_{i i} / 2\right) \geq 0$ for all six possible pairs $i, j$ (with $b_{i j}=b_{j i}$ ). In the spirit of the discussion above for two $\mathrm{U}(1)$ factors, such a $\mathrm{U}(1)^{3}$ model can be realized by Higgsing a model with gauge group $G_{(3)}=\mathrm{SU}(2)^{3} \times \mathrm{SU}(3)^{3}$, where the $\mathrm{U}(1)$ factors are embedded as shown in table 3. Roughly speaking, the $\mathrm{SU}(2)$ factors are associated with the anomaly coefficient combinations $b_{i i} / 2+\sum_{j} b_{i j}$ for each $i$, and the $\mathrm{SU}(3)$ factors are associated with the $-b_{i j}, i \neq j$. With such an embedding, it is straightforward to see that the $\mathrm{SU}(3)$ adjoint and bifundamental fields realize $\mathrm{U}(1)$ charges in the canonical set listed in table 2 . Thus, when all these signs are consistently chosen in this way for the anomaly coefficients, we should realize this canonical set of generic matter fields (for sufficiently small $b$, as usual). For example, when $T=0$, this corresponds to the case where all $b_{i j}$ are negative.

For simplicity, let us continue to consider the case where $T=0$, so the anomaly coefficients are integers. We assume all $b_{i j}$ are nonzero; when one or more of these coefficients vanish, the configuration is degenerate and missing some generic matter fields. From the preceding Higgsing construction, we can consider three other classes of models that are realized by implementing the change of signs (3.32) for each of the three choices of $i$. This will give a total of four possible minimal sets of generic matter fields that we expect to be associated with moduli space branches of maximal dimension; these four sets are given by the canonical set listed in table 2 and the sets realized by flipping the signs of $q_{i}$ in all charges for each choice of $i$. For $T=0$, where the $b_{i j}$ are integers, these four classes of models correspond to sets of $b_{i j}$ where an even number of these anomaly coefficients are positive. In analogy with a spin model where $b_{i j}$ gives the coupling between pairs of spins, we refer to this as a "non-frustrated" configuration. In these cases, the type $(1, \pm 1, \pm 1)$ matter choices in generic models are also fixed by the anomaly coefficient signs. It is interesting to also consider the four "frustrated" configurations of $b_{i j}$, where an odd number of the off-diagonal abelian anomaly coefficients are positive. In these cases, again the spectrum of $(2, \pm 1)$ type matter is determined from the sign of the $b_{i j}$ (or in models with $T>0$ by the signs of $\left.b_{i j} \cdot\left(a+b_{i i} / 2\right)\right)$. The spectrum of $(1, \pm 1, \pm 1)$ type fields is, however, not determined uniquely from the signs of the anomaly coefficients in these cases. Roughly speaking, the choice of $(1, \pm 1, \pm 1)$ type fields follows whichever nonfrustrated configuration is "closest" to the given frustrated configuration. For example, with anomaly coefficients $b_{i i}=8, b_{12}=-2, b_{13}=-2, b_{23}=1$, we get the canonical set of $(1, \pm 1, \pm 1)$ fields, while with $b_{i i}=8, b_{12}=-2, b_{13}=-1, b_{23}=2$, which have the same signs, we get the set of generic matter fields $(1,1,1),(1,-1,-1),(1,-1,1)$ associated with 


\begin{tabular}{|l|ccc|}
\hline & $\mathrm{U}(1)$ & $\mathrm{U}(1)$ & $\mathrm{U}(1)$ \\
\hline $\mathrm{SU}(2)$ & $\left(\begin{array}{ll}0 & 0 \\
0 & 0\end{array}\right)$ & $\left(\begin{array}{ll}0 & 0 \\
0 & 0\end{array}\right)$ & $\left(\begin{array}{cc}1 & 0 \\
0 & -1\end{array}\right)$ \\
$\mathrm{SU}(2)$ & $\left(\begin{array}{cc}0 & 0 \\
0 & 0\end{array}\right)$ & $\left(\begin{array}{cc}1 & 0 \\
0 & -1\end{array}\right)$ & $\left(\begin{array}{ll}0 & 0 \\
0 & 0\end{array}\right)$ \\
$\mathrm{SU}(2)$ & $\left(\begin{array}{cc}1 & 0 \\
0 & -1\end{array}\right)$ & $\left(\begin{array}{cc}0 & 0 \\
0 & 0\end{array}\right)$ & $\left(\begin{array}{ll}0 & 0 \\
0 & 0\end{array}\right)$ \\
$\mathrm{SU}(3)$ & $\left(\begin{array}{ccc}0 & 0 & 0 \\
0 & 0 & 0 \\
0 & 0 & 0\end{array}\right)$ & $\left(\begin{array}{ccc}1 & 0 & 0 \\
0 & -1 & 0 \\
0 & 0 & 0\end{array}\right)$ & $\left(\begin{array}{ccc}0 & 0 & 0 \\
0 & 1 & 0 \\
0 & 0 & -1\end{array}\right)$ \\
$\mathrm{SU}(3)$ & $\left(\begin{array}{ccc}1 & 0 & 0 \\
0 & -1 & 0 \\
0 & 0 & 0\end{array}\right)$ & $\left(\begin{array}{ccc}0 & 0 & 0 \\
0 & 0 & 0 \\
0 & 0 & 0\end{array}\right)$ & $\left(\begin{array}{ccc}-1 & 0 & 0 \\
0 & 0 & 0 \\
0 & 0 & 1\end{array}\right)$ \\
$\mathrm{SU}(3)$ & $\left(\begin{array}{ccc}-1 & 0 & 0 \\
0 & 0 & 0 \\
0 & 0 & 1\end{array}\right)$ & $\left(\begin{array}{ccc}0 & 0 & 0 \\
0 & 1 & 0 \\
0 & 0 & -1\end{array}\right)$ & $\left(\begin{array}{lll}0 & 0 & 0 \\
0 & 0 & 0 \\
0 & 0 & 0\end{array}\right)$ \\
\hline
\end{tabular}

Table 3. Embedding of $\mathrm{U}(1)^{3}$ into $\mathrm{SU}(2)^{3} \times \mathrm{SU}(3)^{3}$. The generator of a given $\mathrm{U}(1)$ factor is the sum of the $\mathrm{SU}(N)$ generators in the corresponding column.

positive choices of $b_{13}, b_{23}$ (which can be related to the canonical set of generic matter fields by flipping the sign on $\left.q_{3}\right)$.

To conclude this section, we consider how the anomaly-equivalent representations $(\square, \square, \mathbf{1})$ and $(\square, \bar{\square}, \mathbf{1})$ of $\mathrm{SU}(3)^{3} \subset G_{(3)}$ behave under Higgsing to $\mathrm{U}(1) \times \mathrm{U}(1)$. As discussed above, with the embedding of table 3 , the fundamental-fundamental matter field gives rise to only the canonical choices of generic matter fields. On the other hand, the fundamental-antifundamental field gives rise to charges including $(-1,-1,2)$. (Note that such a charge can also arise from fundamental-fundamental matter fields when the signs on the embedding are chosen differently). While this may seem to suggest that $(2,-1,-1)$ type matter fields may arise in generic $\mathrm{U}(1)^{3}$ models, this is not the case. Comparing the complete sets of fields that arise from the fundamental-fundamental and fundamentalantifundamental $\mathrm{SU}(3) \times \mathrm{SU}(3)$ representations, we find that the resulting sets of $\mathrm{U}(1)^{3}$ fields have an anomaly equivalence

$$
\begin{gathered}
(2,-1,-1)+(2,0,0)+(1,1,-1)+(1,-1,1)+(1,-1,0) \\
+(1,0,-1)+(0,1,1)+(0,1,0)+(0,0,1) \\
\longleftrightarrow \quad \\
\quad(2,0,-1)+(2,-1,0)+(-1,1,1)+(1,1,-1)+(1,1,0) \\
\quad+(1,0,1)+2 \times(0,1,-1)+(0,0,0)
\end{gathered}
$$


This illustrates several things: first, it gives a simple understanding of the anomaly equivalence from $(2,-1,-1)$ to canonical generic matter; second, it is an example of how Higgsing matter that is anomaly-equivalent to generic matter can give non-generic matter fields; third, it emphasizes the point that while they are anomaly-equivalent, the representations $(\square, \square)$ and $(\square, \square)$ of $\mathrm{SU}(N) \times \mathrm{SU}(M)$ behave physically quite differently in many circumstances.

\subsection{Generic matter with more than three $U(1)$ factors}

For gauge groups with more than three $\mathrm{U}(1)$ factors, there are still further ambiguities in what defines generic matter. For a gauge group $\mathrm{U}(1)^{s}$ with $s>3$, in addition to the generic matter charge types $(0),(1),(2),(1, \pm 1),(2, \pm 1)$ and $(1, \pm 1, \pm 1)$ that can arise with a gauge group of $\mathrm{U}(1)^{3}$, among generic matter charge types we must also include fields with charges $(1, \pm 1, \pm 1, \pm 1)$ for any subset of four of the $\mathrm{U}(1)$ factors. Based on the structure of $\mathrm{U}(1)^{3}$ generic matter and on counting of anomaly cancellation conditions, in any given generic $\mathrm{U}(1)^{s}$ model we might expect that for each subset of four $\mathrm{U}(1)$ factors only one of the possible eight of these charge types will appear for any given set of anomaly coefficients. This turns out not to be the case, however, as we now describe in further detail.

From the point of view of counting anomaly constraints, we see that eq. (2.11d) gives an additional $\left(\begin{array}{l}s \\ 4\end{array}\right)$ constraints beyond those considered in the analysis for up to three $\mathrm{U}(1)$ factors, so including one new charge type for each set of four $\mathrm{U}(1)$ factors would give a set of generic matter fields that matches the number of $\mathrm{AC}$ equations, as we might desire for the subset of generic matter types realized in any given generic model. We can, however, identify anomaly equivalences that exchange any field of charge type $(1, \pm 1, \pm 1, \pm 1)$ for any other field of another such charge type under the same set of four U(1) factors without changing the number of uncharged scalars, assuming sufficient quantities of all other generic matter fields. Restricting attention to the simplest case of $\mathrm{U}(1)^{4}$, we have for example the anomaly equivalence

$$
\begin{aligned}
& (1,-1,1,-1)+(-1,1,1,0)+(1,0,-1,1)+(1,-1,0,1)+(0,1,1,-1) \\
& +(1,0,0,-1)+(0,1,-1,0)+(0,1,0,1)+(1,0,1,0) \\
& \longleftrightarrow \\
& (1,-1,-1,1)+(1,0,1,-1)+(1,-1,1,0)+(0,1,-1,1)+(-1,1,0,1) \\
& +(0,1,0,-1)+(1,0,-1,0)+(1,0,0,1)+(0,1,1,0) .
\end{aligned}
$$

This anomaly equivalence suggests that there can be multiple anomaly-equivalent models with different subsets of the generic matter fields; furthermore, we may expect to see "generic models" of maximal moduli space dimension that contain more distinct matter charges than the appropriate number of $\mathrm{AC}$ equations. In fact, this seems to indeed be possible, and can be understood in relation to the anomaly equivalence of the fundamentalfundamental and fundamental-antifundamental fields for an $\mathrm{SU}(N) \times \mathrm{SU}(M)$ gauge group.

To explore this further, we can take an embedding of $\mathrm{U}(1)^{4}$ into $G_{(4)}=\mathrm{SU}(2)^{4} \times \mathrm{SU}(3)^{6}$, analogous to that given in table 3 for $\mathrm{U}(1)^{3}$. The charges resulting from the Higgsing of such a model with generic matter for the nonabelian gauge group are of the forms (0), (1), 
(2), (1, 1), (1, -1), (2,-1), (1,1,-1), (1,1,-1,-1). (Note that other choices of embedding, or including fundamental-antifundamental fields in the nonabelian theory can give rise to charges type $(1, \pm 1, \pm 1, \pm 1)$ with different sign choices. Other embedding choices can also give rise to charges of type $(2, \pm 1, \pm 1)$, but these can be removed in favor of the generic matter types using the anomaly equivalence (3.44).) While this Higgsing construction gives rise to a set of $\mathrm{U}(1)^{4}$ models with only generic matter types, it generally will give more than one set of $(1,1,-1,-1)$ type charges. While we can exchange one such charge for another through eq. (3.45), this does not change the number of uncharged scalar fields, so this construction gives models with more distinct generic matter charge types than expected from the number of AC equations, although these models should be generic in the sense of maximizing moduli space dimension. Indeed, from the point of view of this Higgsing process, the equivalence (3.45) can be associated with the Higgsed version of the anomaly equivalence between fundamental-fundamental and fundamental-antifundamental $\mathrm{SU}(3) \times \mathrm{SU}(3)$ representations. This can be further understood, in particular, by noting that the l.h.s. and r.h.s. of the anomaly equivalence are related by simply flipping the signs on the last two charges. Thus, we see that at four (or more) U(1) factors, just in the same way as for multiple nonabelian factors, the number of distinct types of generic matter fields realized in any given generic model can exceed the number of anomaly cancellation equations.

Combining this analysis with our understanding of generic matter with three or fewer nonzero abelian charges, we can summarize the situation for the generic matter spectrum of a general $\mathrm{U}(1)^{s}$ theory: the bi-charged matter under any pair of $\mathrm{U}(1)$ factors will be determined just as in the $\mathrm{U}(1)^{2}$ and $\mathrm{U}(1)^{3}$ cases from the anomaly coefficients, with in particular the types of $(2, \pm 1)$ matter being determined by the signs of the terms $b_{i j} \cdot(a+$ $\left.b_{i i} / 2\right)$. Similar to the analysis of $\mathrm{U}(1)^{3}$ models in the previous subsection, we can consider each $\mathrm{U}(1)^{3}$ subgroup of $\mathrm{U}(1)^{k}$ to be "frustrated" or "non-frustrated" depending on the signs of the relevant $b_{i j}$ anomaly coefficients, and the spectrum of $(1, \pm 1, \pm 1)$ matter will be correlated to these anomaly coefficients for each $\mathrm{U}(1)^{3}$ subgroup as discussed above. For 4-charged matter of the types $(1, \pm 1, \pm 1, \pm 1)$, however, we may generally expect to find models with different combinations of matter charges even for fixed anomaly coefficients; such models will be related by anomaly equivalences such as eq. (3.45). While for certain combinations of anomaly coefficients we expect only certain types of $(1, \pm 1, \pm 1, \pm 1)$ to be possible in generic models, we leave a systematic analysis of the constraints and possibilities for further work.

\subsection{Other nonabelian factors}

The analysis of other nonabelian factors is closely parallel to that of $\mathrm{SU}(N)$. While we have not carried out this analysis at the same level of detail as with $\mathrm{SU}(N)$, we can enumerate the types of representations we expect to find as generic for the other compact simple Lie groups. Namely, for $\operatorname{Sp}(N)$ we expect to find fundamentals, adjoints, and antisymmetric representations, as for $\mathrm{SU}(N)$, while for $\mathrm{SO}(N)$ we expect to find fundamentals, spinors, and adjoints. The exceptional groups have no quartic Casimir, and so behave like $\mathrm{SU}(2)$ and $\mathrm{SU}(3)$; thus, we expect only fundamentals and adjoints to appear generic for these groups. (For the exceptional groups the relevant fundamental representations are the smallest- 
dimensional nontrivial representations, i.e., the $\mathbf{7}$ of $\mathrm{G}_{2}, \mathbf{2 7}$ of $\mathrm{E}_{6}$, etc.). Note that for $\mathrm{E}_{8}$, there are not distinct fundamental and adjoint representations, and so there appears to be a counting mismatch with the AC conditions; however, we do not expect to find matter in $6 \mathrm{D}$ supergravity theories with gauge group $\mathrm{E}_{8}$, and so this is not an issue.

We expect for each of these nonabelian gauge factors that the representations listed play the same role as the analogous representations for $\mathrm{SU}(N)$, with in particular matter charged under multiple fields including a given nonabelian factor involving the fundamental in the same way as for $\mathrm{SU}(N)$. Thus, for example, for $\mathrm{G}_{2} \times \mathrm{SU}(2)$, generic matter includes bi-charged matter in the $(\mathbf{7}, \mathbf{2})$ representation (which is self-conjugate, and so can appear in half-hypermultiplets).

\section{Generic matter in F-theory and the string swampland}

From the point of view of F-theory, the generic matter representations described in section 3.1 arise naturally through the simplest Weierstrass model constructions of both nonabelian and abelian gauge groups. Other "exotic" matter representations require more complicated constructions that involve more singular geometries and tuning additional moduli. The framework of F-theory also provides explicit constructions of "matter transitions," which exchange one kind of matter fields for another anomaly-equivalent set of matter fields without changing the gauge group. We summarize here how the models constructed in F-theory fit into the framework of generic matter defined earlier. In section 4.1, we describe F-theory models with $\mathrm{SU}(N)$ gauge factors only; F-theory models with other nonabelian gauge factors besides $\mathrm{SU}(N)$ can be constructed in a similar fashion to the simplest $\mathrm{SU}(N)$ models (using Tate tunings), and similarly give rise to generic matter fields. In section 4.2, we describe F-theory models with one or more U(1) factors.

F-theory gives rise to most $6 \mathrm{D}$ supergravity theories that can be realized in any version of string theory. ${ }^{4}$ In section 4.3 , we describe how generic matter provides a helpful paradigm with which to explore the "swampland" of $6 \mathrm{D}$ models that appear acceptable from anomaly cancellation and other known quantum consistency conditions, but which are not realized in F-theory.

\subsection{Tate and Weierstrass models for $\mathrm{SU}(N)$ gauge groups}

We review briefly the basic elements of F-theory that are involved in constructing a $6 \mathrm{D}$ theory with a gauge group $\mathrm{SU}(N)$. For a more extensive background on F-theory see the original papers [31-33] or the reviews [34, 35].

An F-theory model of a $6 \mathrm{D}$ supergravity theory is described by an elliptically fibered Calabi-Yau threefold encoded in a Weierstrass model

$$
y^{2}=x^{3}+f x+g,
$$

where $f, g$ are functions (or more properly sections of certain line bundles) on a complex surface base $B$. The geometry of the Calabi-Yau threefold encodes the physics of the

\footnotetext{
${ }^{4}$ Some exceptions include models that can be realized in the "frozen" phase of F-theory [6], or which include exotic matter that cannot be realized in conventional F-theory [29, 30].
} 
corresponding $6 \mathrm{D}$ theory; the number of tensor multiplets is given by $T=h^{1,1}(B)-1$, the $6 \mathrm{D}$ string charge lattice is given by $\Gamma=H^{1,1}(B, \mathbb{Z})$, and the anomaly coefficient $a$ is the element in $\Gamma$ associated with the canonical class $K_{B}$ of the base. The gauge group and matter fields are encoded in singularities of the elliptic fibration.

The F-theory model gives an $\mathrm{SU}(N)$ gauge group over a divisor (complex codimension one locus) $\sigma=0$ when the discriminant

$$
\Delta=4 f^{3}+27 g^{2}
$$

vanishes to order $N$ over $\sigma$, while either $f$ or $g$ does not vanish over $\sigma$. In general, this condition requires a precise cancellation of the terms in the discriminant at each order in $\sigma$. The simplest way that these cancellations can be imposed is through a "Tate form" model $[36,37]$

$$
y^{2}+a_{1} x y z+a_{3} y z^{3}=x^{3}+a_{2} x^{2} z^{2}+a_{4} x z^{4}+a_{6} z^{6} .
$$

When eq. (4.3) is converted into Weierstrass form by completing the square and clearing the quadratic term in $x$ (and setting $z=1$ ), an $\mathrm{SU}(N)$ singularity is realized over the divisor $\sigma=0$ when the $a_{n}$ coefficients vanish to the orders $\left[a_{1}, a_{2}, a_{3}, a_{4}, a_{6}\right]=$ $[0,1,\lfloor N / 2\rfloor,\lfloor(N+1) / 2\rfloor, N]$.

This simple Tate form (4.3) for $\mathrm{SU}(N)$ gives rise to a $6 \mathrm{D}$ theory where the $\mathrm{SU}(N)$ anomaly coefficient is the class in $\Gamma=H^{1,1}(B, \mathbb{Z})$ associated with the divisor $\sigma(b=[\sigma])$, and $\mathrm{SU}(N)$ matter fields transform under the fundamental, adjoint, and two-index antisymmetric matter representations - precisely the generic matter types listed in table 2 . This can be seen geometrically in the F-theory picture from the fact that adjoint representations arise from the genus of a smooth divisor, and the fundamental and two-index antisymmetric matter representations arise from simple codimension-two singularities associated with points on $\sigma$ where $\Delta$ vanishes to orders higher than $N$ through a simple singularity enhancement described using the Kodaira classification as $A_{N-1} \rightarrow A_{N}, A_{N-1} \rightarrow D_{N}$, respectively. The Tate form construction of $\mathrm{Sp}(N)$ and $\mathrm{SO}(N)$ gauge groups proceeds in a similar fashion, and for exceptional groups the Tate construction is even simpler, involving only conditions on the orders of vanishing of $f$ and $g$.

Any other kind of matter representation for $\mathrm{SU}(N)$ (or any other nonabelian gauge factor) in F-theory requires some additional more special tuning of the Weierstrass coefficients $f, g$. There is only one case in which it is known that this can be achieved through a Tate-type tuning: it was shown in [38] that a special Tate tuning of $\mathrm{SU}(6)$ can be realized when the $a_{n}$ coefficients vanish to orders $[0,2,2,4,6]$ (instead of the usual Tate SU(6) tuning $[0,1,3,3,6])$. In this case, at the locus $a_{1}=\Delta=0$ there arise higher singularities associated with the three-index $\mathbf{2 0}$ representation of $\mathrm{SU}(6)$. Since $a_{4}$ generally has at least as many degrees of freedom at order $\sigma^{3}$ as $a_{3}$ does at order $\sigma^{2}$, and $a_{2}$ generally also has degrees of freedom at order $\sigma$, it turns out that this exotic Tate tuning always reduces the number of degrees of freedom in the model, in accord with the principle that exotic matter always reduces the number of degrees of freedom (uncharged matter hypermultiplets) in the theory.

A systematic approach to tuning $\mathrm{SU}(N)$ Weierstrass models by arranging an orderby-order cancellation in the terms of the expansion of $\Delta$ in terms of expansions of $f, g$ as 
power series in $\sigma$ was carried out in [39]. In that paper, the assumption was made that $\sigma$ is a smooth divisor, and that the ring of functions on $\sigma$ is a Unique Factorization Domain (UFD). From this point of view, the three-index antisymmetric tensor representations of not only $\mathrm{SU}(6)$ but also $\mathrm{SU}(7)$ and $\mathrm{SU}(8)$ can be realized explicitly as tuned Weierstrass models. In each case, it can be seen explicitly that more degrees of freedom must be tuned away (associated with a decrease in the number of uncharged hypermultiplets) to realize the three-index antisymmetric tensor representations.

A further study of exotic matter was carried out in [40]. In that paper it was shown explicitly how the two-index symmetric representation of $\mathrm{SU}(N)$ and the three-index symmetric representation of $\mathrm{SU}(2)$ can be realized through a non-UFD tuning of $\mathrm{SU}(N)$ on a singular divisor $\sigma$. Again, in these situations, tuning the curve $\sigma$ so that it is singular and the spectrum includes exotic matter representations removes degrees of freedom encoded in uncharged hypermultiplets. In that paper, it is also argued that these are the only exotic representations of $\mathrm{SU}(N)$ that can be realized in conventional F-theory. Furthermore, for other gauge factors including in particular the exceptional gauge algebras, the same argument shows that no other non-generic matter parameterization should be possible in conventional F-theory other than for $\mathrm{SU}(N)$ and closely related $\mathrm{Sp}(N)$ cases.

Note that, as discussed in [40], although exotic matter representations can generally only be achieved by fine-tuning from a model with only generic matter, there are cases in which models with generic matter are ruled out by anomaly cancellation considerations that would imply that some matter representations have negative multiplicity, as mentioned in section 3.4. In such situations, there can be F-theory models that realize a given gauge group on a divisor $\sigma$ of a certain class with exotic matter, where no generic matter model is possible. In general, this occurs precisely where the anomaly coefficient $b$ becomes too large for generic matter to be possible with non-negative multiplicities.

As an example of how F-theory realizes generic $\mathrm{SU}(N)$ matter through Tate tunings at small $b$, we consider the simplest case of the base $\mathbb{P}^{2}$, corresponding to $T=0$. In this case, homology classes in $H^{1,1}\left(\mathbb{P}^{2}\right)$ are given by integer multiples of the generating class $H$, so anomaly coefficients are simply denoted by integers. The anomaly coefficient $-a=3$ corresponds to the anticanonical class $-K=3 H$. A Tate tuning of $\mathrm{SU}(2)$ is possible up to $b=12$, giving the generic matter $\mathrm{SU}(2)$ models listed in eq. (3.18). For $b>12$, any F-theory model must be described by a more general class of Weierstrass model that does not take the Tate form; in particular, as described in [40], the divisor $\sigma$ must have a triple-point singularity for each (half-hyper) matter multiplet in the $\square \square \square$ representation. While there is no completely systematic construction of such models, some F-theory models of this type with $b>12$ are explicitly constructed in [41]; for example (see table 3.22 in that paper) there is a model with $b=13$ that has 6 triple-symmetric matter fields, associated with 12 triple-point singularities in the locus $\sigma=0$. The F-theory construction of such exotic matter models is discussed further in section 4.3.

For $\mathrm{SU}\left(N_{1}\right) \times \cdots \times \mathrm{SU}\left(N_{r}\right)$ theories, standard F-theory constructions using the Tate construction for each factor yield the generic representations of the form $1, \square, \operatorname{Adj}, \square$, $(\square, \square)$, and $(\square, \square)$. Some more exotic representations, such as $(\square, \square)$, can also be 
constructed with a reduction in the number of uncharged scalars, though the Weierstrass models for such exotic multiply-charged have not been systematically described.

It is interesting to consider how the anomaly-equivalent generic matter representations $(\square, \bar{\square})$ and $(\square, \square)$ arise in simple F-theory models. While determining which of these representations arises at a given singularity involves somewhat subtle global aspects of a given Weierstrass model [39], it is straightforward to see that many general Tate models for $\mathrm{SU}(N) \times \mathrm{SU}(M)$ will include both types of representations, using a simple Higgsing argument. Consider, for simplicity, models with no tensor multiplets, corresponding to F-theory on $\mathbb{P}^{2}$. We can perform a Tate tuning of $\mathrm{SU}(N)$ on a smooth curve of degree $d$, which has self-intersection $n=d^{2}$ and genus $g=(d-1)(d-2) / 2$. The resulting $\operatorname{SU}(N)$ model has generic matter content

$$
g \times \operatorname{Adj}+[16(1-g)+(8-N) n] \times \square+(n+2-2 g) \times \square .
$$

We can break $\mathrm{SU}(N) \rightarrow \mathrm{SU}(N-k) \times \mathrm{SU}(k)$ by Higgsing on a pair of antisymmetric matter fields. The resulting model has the spectrum we expect from a Tate tuning of the product gauge group on a pair of distinct divisors of degree $d$. Under this Higgsing process, the decomposition of the $\square$ representations of $\mathrm{SU}(N)$ includes bifundamental representations $(\square, \square)$, while the decomposition of the adjoints of $\mathrm{SU}(N)$ includes $(2 \times)$ fundamentalantifundamental matter representations $(\square, \square)$. Thus, the resulting $\mathrm{SU}(N-k) \times \mathrm{SU}(k)$ model contains, among other fields, the matter representations

$$
2 g \times(\square, \bar{\square})+\left(d^{2}-2 g\right)(\square, \square) .
$$

As expected from anomaly cancellation, the total number of fields in these two representations sums to $d^{2}$, which is the number of points where the two degree $d$ curves intersect; however, the spectrum includes $d^{2}-3 d+2$ fundamental-antifundamental matter fields and $3 d-2$ bifundamental fields. This is a simple example of how F-theory generically gives rise to a mixture of the different anomaly-equivalent generic matter representations in cases where there are more generic matter fields than anomaly cancellation equations.

\section{$4.2 \mathrm{U}(1)$ matter in F-theory}

F-theory constructions of $6 \mathrm{D}$ models with abelian gauge groups are not well understood beyond the simplest models with a single $\mathrm{U}(1)$ factor and the generic $q=1,2$ matter representations. We summarize briefly here the situation of what is known. The generic matter analysis for $\mathrm{U}(1)^{2}, \mathrm{U}(1)^{3}$, and $\mathrm{U}(1)^{s}, s>3$ models carried out above gives new insights into the structure of F-theory constructions in these cases.

\subsubsection{One $U(1)$ factor}

A general construction of an F-theory model over a given base $B$ with a single U(1) factor was given by Morrison and Park in [42]. In the Morrison-Park model, the only matter fields that arise are the generic charges $q=1,2$. Thus, again, in this case the simplest F-theory construction gives a model with only generic charges. 
There are only a limited set of explicit Weierstrass model constructions known that give rise to $\mathrm{U}(1)$ theories with charges $q>2$. A specific set of models with $q=3$ was first identified in [43]. This set was generalized to a more general class of models with $q=3$ charges and related to non-UFD singularities in [44]; in this paper, Raghuram also identified a limited class of models with charge $q=4$. At this time, there are no explicit Weierstrass model constructions known for any $\mathrm{U}(1)$ charges $q>4$, although general arguments from Higgsing known nonabelian models suggest that there should be F-theory models with $\mathrm{U}(1)$ charges $q=5,6$ that arise from Higgsing simple Tate $\mathrm{SU}(N)$ models over the base $B=\mathbb{P}^{2}[5]$ and F-theory models with $\mathrm{U}(1)$ charges up to $q=21$ at least that arise from Higgsing more exotic nonabelian models on other bases [45]. A recent paper [46] gives an alternative construction of F-theory models with charge $q=4$ matter through a weak coupling limit of type IIB string theory, and identifies IIB models that should correspond to charge $q=5,6$ matter.

\subsubsection{Two $\mathrm{U}(1)$ factors}

For models with two $\mathrm{U}(1)$ factors $(G=\mathrm{U}(1) \times \mathrm{U}(1))$, a rather general construction was put forth in [28], following some more specific constructions with more constrained matter content $[47-50]$. The matter content of the general $\mathrm{U}(1)^{2}$ construction matches well with the generic $\mathrm{U}(1) \times \mathrm{U}(1)$ matter content described above, and can be understood as coming from the Higgsing of a nonabelian model with gauge group $G_{(2)}=\mathrm{SU}(2) \times \mathrm{SU}(2) \times \mathrm{SU}(3)$. Here, the $\mathrm{U}(1)$ factors are associated with divisors $A C, B C$, where the nonabelian factors are supported on $A, B, C$; this leads to a natural embedding of the $\mathrm{U}(1)$ factors in the Cartan subalgebra of the nonabelian group as in eq. (3.38). The explicit analysis of the $\mathrm{U}(1) \times \mathrm{U}(1)$ Weierstrass model in [28] was shown to give the generic matter spectrum described in section 3.6 with charges $(2,1)$ and $(1,2)$. This setup should also allow for the construction of models with generic matter charges $(2,-1)$ and $(1,-2)$, with different choices of divisors associated with the anomaly coefficients $b_{i j}$, though that is not addressed directly in the paper; we leave an investigation of the details of this analysis for further work. One interesting feature of the construction in [28] is that the most general model there automatically includes non-generic charge $(2,2)$ matter. A slightly more restricted version of the model gives only the generic matter charges in table 2 .

\subsubsection{Three or more U(1) factors}

At this point there is very little understanding of explicit F-theory Weierstrass models for theories with more than two U(1) factors. In [51], a model was given with three $\mathrm{U}(1)$ factors, but it provides only a very limited class of spectra and does not contain all expected generic matter fields or generic models. In [52], explicit Weierstrass models were given for K3 surfaces of Mordell-Weil rank up to 4, but the matter spectra of F-theory compactifications on these surfaces was not explored. There are also some explicit 6D models known with Mordell-Weil rank up to 8 and no matter charged under the U(1) factors (i.e., non-Higgsable U(1) factors) [53, 54]. These models are rather special. We can, however, from the analysis of generic matter, gain some insight into the structure one might expect for a general F-theory construction of generic $\mathrm{U}(1)^{s}$ models. 
Following the structure of the general $\mathrm{U}(1)^{2}$ F-theory model, it was conjectured in [28] that a general class of $\mathrm{U}(1)^{s}$ models could be constructed from the Higgsing of a model with gauge group

$$
G=\mathrm{SU}(2)^{s} \times \mathrm{SU}(3)^{\left(\begin{array}{c}
s \\
2
\end{array}\right)} \times \mathrm{SU}(4)^{\left(\begin{array}{c}
s \\
3
\end{array}\right)} \times \cdots \times \mathrm{SU}(s)^{s} \times \mathrm{SU}(s+1),
$$

in which each $\mathrm{U}(1)$ has a component in one of the $\mathrm{SU}(2)$ factors, each pair of $\mathrm{U}(1) \mathrm{s}$ has a common component in one of the $\mathrm{SU}(3)$ factors, and so on. From the analysis of generic matter in $\mathrm{U}(1)^{s}$ models above, however, and the structure of the anomaly equations, it seems that a broad class of generic matter models can be realized from Higgsing nonabelian models with the simpler gauge group

$$
G_{(s)}=\mathrm{SU}(2)^{s} \times \mathrm{SU}(3)^{\left(\begin{array}{c}
s \\
2
\end{array}\right)} .
$$

Here, there is an $\mathrm{SU}(2)$ factor associated with each $\mathrm{U}(1)$ factor, and an $\mathrm{SU}(3)$ factor associated with each pair of $\mathrm{U}(1)$ factors. We can always construct an embedding of $\mathrm{U}(1)^{s}$ into this gauge group analogous to the embedding of $\mathrm{U}(1)^{3}$ into $G_{(3)}=\mathrm{SU}(2)^{3} \times$ $\mathrm{SU}(3)^{3}$ given in table 3 . Higgsing a $G_{(s)}$ model with generic matter to $\mathrm{U}(1)^{s}$ using such an embedding gives a spectrum with charges of the generic matter types described in the previous section.

While it is straightforward to construct an F-theory model with generic matter and gauge group $G_{(s)}$ with any desired (relatively small) anomaly coefficients using the Tate construction, unfortunately it is not clear given such a Weierstrass model how to perform a Higgsing. The existence of the nonabelian model guarantees that there should be a Weierstrass model for the resulting Higgsed $\mathrm{U}(1)^{s}$ model, which with an appropriate embedding will have generic matter fields as described in sections 3.7 and 3.8. Unfortunately we do not know how to compute this model explicitly. One approach would be to simultaneously tune Morrison-Park type $\mathrm{U}(1)$ factors on the relevant combinations of $\mathrm{SU}(2)$ and $\mathrm{SU}(3)$ divisors, but it is not clear even in the case of $\mathrm{U}(1)^{2}$ how to derive the general model of [28] from this point of view. Indeed, understanding high-rank abelian gauge groups associated with large rank Mordell-Weil groups in the F-theory geometry is an open problem that deserves further attention; hopefully the structure of generic matter analyzed here may help provide some insights into these problems.

\subsection{Generic matter, string universality, and the swampland}

The concept of "generic" matter representations in 6D supergravity theories is a useful tool in analyzing the relationship between low-energy constraints on supergravity theories and UV complete models that come from F-theory or other string compactifications. It was conjectured in [55] that "string universality" may hold for $6 \mathrm{D} \mathcal{N}=(1,0)$ supergravity theories, meaning that every massless $6 \mathrm{D}$ spectrum of tensor fields, gauge fields, and matter representations that can be consistently coupled to quantum gravity is realized in string theory. Currently, there is still a significant gap in understanding whether this is true; in particular, even for theories with no tensor multiplets there is an infinite family of $U(1)$ charge spectra that are consistent with anomalies and other known low-energy consistency 
conditions but are not realized in F-theory [5]. The set of theories that satisfy known quantum consistency conditions but that are not realized by known string theory constructions has been dubbed the "swampland" $[56,57] . .^{5}$ At least for theories with generic matter types, it seems that some simple assumptions about the structure of the low-energy theory suffice to limit the swampland almost completely. This essentially focuses general questions about string universality and the swampland to more specific questions about exotic matter and some subtle issues related to the positivity cone of the low-energy theory. We summarize here briefly the general setup and explain how the swampland is substantially reduced for theories with generic matter.

\subsubsection{Structure of $6 \mathrm{D}$ supergravity theories}

Elaborating further on the basic framework described in section 2 , a $6 \mathrm{D} \mathcal{N}=(1,0)$ supergravity theory is described by some basic data for the low-energy theory. Any such theory has one gravity (super)multiplet, $T$ tensor multiplets, a gauge group $G$ associated with $\operatorname{dim}(G)$ gauge multiplets, and $H$ matter hypermultiplets that transform under various representations of the group $G$. In addition to this structure, there is a signature- $(1, T)$ integer lattice $\Gamma$, often referred to as the "anomaly lattice," which must be unimodular [27]. There is a gravitational anomaly coefficient $a \in \Gamma$, which satisfies $a \cdot a=9-T$ by the gravitational anomaly condition. For each nonabelian factor of the gauge group there is an anomaly coefficient $b_{\kappa} \in \Gamma$, and for each pair of abelian factors there is an anomaly coefficient $b_{i j} \in \Gamma$. These anomaly coefficients satisfy the anomaly constraints 2.9 and (2.11), which lead to various further integrality constraints [22, 55], including the condition that for a theory with $\mathrm{U}(1)$ gauge factors the anomaly coefficients $b_{i i}$ are even, in the sense that $b_{i i} \in 2 \Gamma$. Finally, there is a positivity cone in the anomaly lattice, corresponding to the Kähler moduli space of the theory. All of these quantities have a direct interpretation in the geometry of F-theory: the lattice $\Gamma$ is the homology lattice of the base surface $B$, the positivity cone is the cone of effective divisors, $a$ corresponds to the canonical class, $b$ corresponds to the divisor class of the seven-branes supporting a given gauge factor, etc.

For each value of $T$, then, we can classify theories beginning with a choice of $a$ and lattice $\Gamma$ with positivity cone. It was shown in [22] (based on some minimal assumptions) that $a$ is a characteristic vector for $\Gamma$, meaning that $a \cdot x+x \cdot x \in 2 \Gamma$ for any $x \in \Gamma$. For small values of $T$, there are only a few possible choices of lattice and $a$, and the characteristic vector condition suffices to limit the possibilities to those that can arise from F-theory. In particular, for $T=0$ the lattice $\Gamma$ is uniquely defined as $\Gamma=(1)$, and $-a=3$ is the unique choice for $a$ (with the sign fixed by the condition that $-a$ lies in the positivity cone, which by convention we take to be the set of positive values in $\Gamma$ ). For $T=1$, there are two possible unimodular lattices: the odd lattice with inner product

$$
\Omega_{1}=\left(\begin{array}{cc}
1 & 0 \\
0 & -1
\end{array}\right)
$$

\footnotetext{
${ }^{5}$ Note that different authors use the term swampland differently. We refer to the swampland as containing any model that does not violate known quantum consistency conditions but does not have a known explicit string construction. Thus, the swampland by this definition is time-dependent and can shrink as new constraints and new string constructions are identified.
} 
and the even lattice with inner product

$$
\Omega_{0}=U=\left(\begin{array}{ll}
0 & 1 \\
1 & 0
\end{array}\right) .
$$

We denote the corresponding string charge lattices by $\Gamma_{1}, \Gamma_{0}$. For $\Gamma_{1}$, the only possible choice for $a$ so that $a \cdot a=9-T=8$ is $-a=-a_{1}=(3,-1)$, up to symmetries. For $\Gamma_{0}$, there are two possibilities: $-a=-a_{0}=(2,2)$ and $-a=-a_{0}^{\prime}=(4,1)$. The second choice is, however, not a characteristic vector, so is not allowed in a consistent low-energy theory. For $T=0,1$, these allowed combinations of the anomaly lattice and $a$ are precisely those allowed by F-theory. In fact, if we assume that the anomaly lattice for any higher value of $T$ has the inner product $\operatorname{diag}(+1,-1,-1, \ldots,-1)$, then it seems that the characteristic vector condition uniquely determines the value $-a=(3,-1,-1, \ldots,-1){ }^{6}$

\subsubsection{Positivity cone and anomaly lattice related swampland issues}

There are several aspects of the anomaly lattice $\Gamma$ and the positivity cone that are not fully understood from the low-energy point of view. In particular, it is not clear how the low-energy theory constrains the positivity cone, and while in F-theory any vector in the positivity cone that has an inner product with itself $x \cdot x<-2$ must support a gauge group $\mathrm{SU}(3)$ or larger [7], this has not been proven purely from consistency considerations arising from coupling the low-energy theory to quantum gravity. These issues lead to various families of swampland theories that cannot be realized in F-theory but do not violate any proven quantum consistency conditions on the low-energy theory. It seems likely that these parts of the swampland can be removed by considering consistency conditions on the worldvolume of strings that couple to the tensor fields [55] (see, e.g., [58-63] for some recent progress in understanding these string world-volume theories). There are thus various important open swampland questions related to the anomaly lattice and positivity cone. Here, however, we assume that the lattice and positivity cone are of a form compatible with F-theory, and consider various gauge groups and matter content in that context.

\subsubsection{The swampland for generic matter}

Restricting attention to anomaly lattices $\Gamma$ and positivity cones compatible with F-theory, and focusing only on theories with the matter fields we have identified as generic, the only questions about theories in the swampland arise from three issues:

(a) large anomaly coefficients $b$,

(b) ambiguities between fundamental-fundamental/fundamental-antifundamental matter for products of nonabelian factors and the related ambiguity associated with the anomaly equivalence (3.45) for theories with more than three $\mathrm{U}(1)$ factors,

(c) a lack of explicit Weierstrass models for generic models with multiple abelian factors.

\footnotetext{
${ }^{6}$ Thanks to Noam Elkies for helpful discussions on this point.
} 
In essentially all of these cases, the appearance of swampland models is associated with our incomplete understanding of how to construct Weierstrass models describing elliptic Calabi-Yau threefolds with sufficiently complicated structure. We now briefly review how the known classes of F-theory constructions described in sections 4.1 and 4.2 match with the set of allowed low-energy theories with generic matter, and where the limitations of these constructions lead us to the swampland.

Recall that for generic matter for any combination of nonabelian and abelian gauge fields, we have found that the anomaly constraints uniquely identify the set of allowed matter representations given the gauge fields and associated anomaly vectors $b_{\kappa}, b_{i j}$, up to the ambiguities in item (b) above. Thus, we can define an apparently consistent low-energy theory by choosing a set of gauge group factors $G_{\kappa}, \mathrm{U}(1)_{i}$ and associated values of $b_{\kappa}, b_{i j}$ that are sufficiently small that the anomaly equations are satisfied by a subset of the set of generic matter fields. In cases with multiple nonabelian factors, or more than three abelian factors, there may be a family of theories associated with choosing different multiplicities of the anomaly-equivalent sets of generic matter fields associated with item (b) above. The swampland question in this context is: for which models in this class does there exist an explicit F-theory construction through a Weierstrass model, or more indirectly an F-theory construction of a model with a larger gauge group that can be Higgsed to the desired model. (There are currently no non-F-theory constructions of models in this class that cannot also be realized by conventional F-theory.)

Many of the theories in this general class can be directly constructed using the Tate form (4.3), as long as the anomaly coefficients are not too large. While there is no direct known Weierstrass construction for generic models with more than two U(1) factors, for small values of the anomaly coefficients as described in sections 3.7 and 3.8 we can realize models with more U(1) factors by Higgsing a Tate model with the appropriate number of $\mathrm{SU}(2)$ and SU(3) factors. These approaches essentially lead to F-theory models for any lowenergy theory that does not suffer from issues (a) and (b), though for multiple $U(1)$ factors the details of the Higgsing construction and associated embeddings have not been worked out in detail for all generic matter combinations. To illustrate the relevant issues more explicitly, we focus first on models with a single gauge factor, where only issue (a) is relevant.

As the simplest example, as discussed in [5], when the gauge group is $\mathrm{SU}(2)$ and $T=0$, there are anomaly-consistent theories for each value $1 \leq b \leq 12$, and all of these theories can be directly constructed as Weierstrass models using the Tate form, so there is no associated swampland for models with only generic matter (except for one subtlety regarding the case $b=12$, which we return to below). The situation is less clear at larger values of $T$. A Tate $\mathrm{SU}(2)$ construction is possible when $b \leq-4 a$, meaning that $-4 a-b$ is in the positivity cone. The condition for generic matter, however, is that $b \cdot b \leq-4 a \cdot b$, which is a weaker condition. Thus, there are some cases that admit generic matter solutions to the anomaly equations, for which a Tate construction is not possible.

As an example, consider the case of the even lattice (4.9), with positivity cone as defined in eq. (3.23); this corresponds to the class of F-theory models compactified on the Hirzebruch surface $\mathbb{F}_{0}=\mathbb{P}^{1} \times \mathbb{P}^{1}$. In this case, $-a=(2,2)$. The anomaly coefficient $b=(2,9)$ (which lies in the shaded but not the hashed region in figure 1 ) is too large 
for a Tate $\mathrm{SU}(2)$ construction, but is compatible with a spectrum $x_{\square}=104, x_{\square} \square=8$ (with 15 uncharged scalars needed to saturate the gravitational anomaly bound). Note that, as guaranteed by $b \in \Gamma_{0}$, this model does satisfy the global anomaly cancellation condition (2.13). This model is thus currently in the swampland, since it satisfies the known quantum consistency conditions (see figure 1) but does not at this time have a known F-theory construction.

While there is no Tate form Weierstrass model for this $\mathrm{SU}(2)$ theory on $\mathbb{F}_{0}$, it is possible that the more general class of non-UFD constructions analyzed in [40] can realize this model. Specifically, this could occur if the genus 8 curve describing the divisor $\sigma$ in the class $b$ where the $\mathrm{SU}(2)\left(A_{1}\right)$ singularity is localized is taken to be singular, so that some of the eight adjoints become localized; in this case the non-UFD ring of functions on $\sigma$ can lead to nontrivial cancellations giving an $\mathrm{SU}(2)$ Weierstrass model that is not in Tate form. We leave a more explicit analysis of this and related situations for future work. Note that the model with anomaly coefficient $b=(1,9)$ is not consistent as it violates the gravitational anomaly bound; since the corresponding curve would be rational (genus 0 ), the non-UFD construction possibility is not an option in this case.

For theories with a single $\mathrm{SU}(N)$ factor more generally, similar issues can arise, giving possible swampland models when the combination $b N$ is too large for a Tate realization. In general, a Tate realization of a theory with gauge group $\mathrm{SU}(N)$ will be possible when $b N \leq-8 a$. When this condition is violated (but the Kodaira bound $b N \leq-12 a$ is still satisfied), there may be Weierstrass constructions even when there is no Tate construction, as in the above $\mathrm{SU}(2)$ example, but there is no general methodology known or general condition for when such a Weierstrass model will exist. Another, slightly more subtle issue can arise when tuning maximal even or odd rank $\mathrm{SU}(N)$ factors through Tate $[25,39,64]$. The simplest example of this is found when trying to construct an $\mathrm{SU}(23)$ or $\mathrm{SU}(24)$ group on a divisor $b=1 \mathrm{in} \mathbb{P}^{2}$. In this case, the Tate tuning of $\mathrm{SU}(23)$ automatically forces a gauge group $\mathrm{SU}(24)$. So, the $T=0, b=1$ model with gauge group $\mathrm{SU}(23)$ is in the swampland; it is not clear if there is a non-Tate F-theory realization of such a model, but it seems unlikely since, unlike in the $\mathrm{SU}(2)$ case mentioned above, the genus of the $b=1$ curve is zero, so there is no opportunity for non-UFD structure. Similar issues arise when tuning, for example, an $\mathrm{SU}(15)$ on $\mathbb{F}_{1}$ or $\mathbb{F}_{2}$.

An even more subtle issue is relevant in the $\mathrm{SU}(24)$ case. In this case, the Tate tuning gives a model with gauge group $\mathrm{SU}(24) / \mathbb{Z}_{2}$. This model has only matter in the two-index antisymmetric tensor representation, which is invariant under the $\mathbb{Z}_{2}$ center. In fact, this seems to be a general pattern: in any model with no massless matter transforming under a central component of the gauge group, the resulting F-theory model has a gauge group that is quotiented by that central component. Since every known F-theory model that we are aware of satisfies this condition, it is natural to make the hypothesis that this is universally true for all F-theory constructions.

For example, with $T=0, b=12$ the $\mathrm{SU}(2)$ model described earlier has only 54 adjoint fields and no fundamentals, and the gauge group is actually $\mathrm{SO}(3)=\mathrm{SU}(2) / \mathbb{Z}_{2}[5,65]$. This is reminiscent of the completeness hypothesis $[66,67]$ stating that any theory coupled to quantum gravity must contain matter transforming in all nontrivial charges under the gauge 
group, but this condition applies only to massless fields and is therefore much stronger. It would be interesting to understand whether this condition is truly a universal constraint; for now, the models like those with a gauge group SU(24) and only two-index antisymmetric matter lie in the swampland.

Turning to models with a only single U(1) factor, again, generic matter content of charges $q=1,2$ is produced in many cases by the Morrison-Park model [42]. As discussed in [5], in the simplest cases where $T=0$, with the allowed values of $a$ and positivity cones compatible with F-theory geometry, the $\mathrm{U}(1)$ anomaly constraints precisely match those for the existence of a Morrison-Park model, and there is no swampland (with the exception of the model with only charge 2 matter that arises from Higgsing the $\mathrm{SU}(2), b=12$ model mentioned above, which violates the general condition discussed there). For larger values of $T$, the anomaly constraints are weaker but, as in the $\mathrm{SU}(2)$ case discussed above, have a seemingly parallel form to the constraints for a Morrison-Park model that would be interesting to understand better. An example of a swampland model here would be the Higgsing of the $b=(2,9), T=1 \mathrm{SU}(2)$ model mentioned above; presumably this model can be realized in F-theory if and only if the corresponding $\mathrm{SU}(2)$ model can also be realized.

Now considering theories with a gauge group containing multiple nonabelian factors, for a gauge group $G=\prod_{\kappa} \mathrm{SU}\left(N_{\kappa}\right)$ in general a Tate realization will be possible if $\sum_{\kappa} b_{\kappa} N_{\kappa} \leq$ $-8 a$, and again there will be cases where this condition is violated but the Kodaira bound $\sum_{\kappa} b_{\kappa} N_{\kappa} \leq-12 a$ is still satisfied where there may be exotic non-UFD or other F-theory constructions. A further issue that arises here is that the Tate construction will give some specific combination of fundamental-fundamental and fundamental-antifundamental matter for each product of nonabelian factors; there will be other anomaly-equivalent models with different multiplicities after exchanging these equivalent representations, and we do not currently have a general approach to analyzing or constructing the sets of models with arbitrary distributions of these anomaly-inequivalent representations. This contributes to parts of the swampland related to issue (b) above.

Finally, the F-theory construction of models with more than two U(1) factors is still not well understood (issue (c) above). For two U(1) factors, the general construction in [28] gives a general class of models with generic matter types. Presumably, like the MorrisonPark model discussed above, there will be cases at $T>0$ where no Tate tuning is possible but we expect a valid spectrum, which will contribute to the swampland of $\mathrm{U}(1)^{2}$ models. As discussed in section 4.2.2, the original analysis of these models made specific positivity choices for the anomaly coefficients, and a complete story would involve generalizing this analysis to all sign choices, though it seems likely this will lead to a consistent construction of all the different classes of $(2, \pm 1)$ matter spectra. For more than two U(1) factors, there is no general explicit model known, so the most general approach available is to implicitly construct $\mathrm{U}(1)^{3}$ and higher rank $\mathrm{U}(1)^{s}$ models by Higgsing generic nonabelian $G_{(s)}=\mathrm{SU}(2)^{s} \times \mathrm{SU}(3)^{\left(\begin{array}{c}s \\ 2\end{array}\right)}$ models. This will give a broad class of generic $\mathrm{U}(1)^{s}$ models, but as for the lower rank models discussed more explicitly, there will likely be similar further components of the swampland. The swampland for $\mathrm{U}(1)^{4}$ and higher models will also contain components from different distributions through the anomaly equivalence (3.45), associated with issue (b) above. 


\subsubsection{Exotic matter and the $6 \mathrm{D}$ swampland}

The concept of generic matter is thus helpful in organizing analysis of questions related to string universality and the swampland for 6D supergravity theories. Going beyond generic matter, the question of which exotic matter types are allowed in F-theory involves much more complex questions of algebraic geometry. For exotic matter charged under nonabelian gauge groups, a systematic analysis of three-index antisymmetric ("genus 0" [17]) SU(N) matter was carried out in [26, 39], and a general methodology for understanding "higher genus" matter representations in terms of singularities over divisors that themselves are singular was developed in [40]. While in many cases there are F-theory models with exotic singularity structures that match with anomaly-free low-energy theories with certain exotic matter content, in other cases it is not known whether F-theory models exist, and in still other cases it is known that F-theory models cannot exist and there are apparently consistent models in the swampland. It may be that some exotic matter is consistent and can be realized in string theory but not conventional F-theory; incorporating such exotic matter such as $E_{7} \times \mathrm{SU}(2)$ bifundamental matter into F-theory may involve the "T-brane" worldvolume fields on 7-branes [30], and other exotic matter may appear in the "frozen phase" of F-theory [6]. For abelian exotic matter, the story is even less clear. As discussed earlier, explicit models for F-theory constructions with abelian charges $q=3,4$ were constructed in $[43,44,46]$, and Higgsing nonabelian constructions can give certain F-theory models with charges up to $q=21$, but there still exists an infinite swampland of higher-charge $\mathrm{U}(1)$ models that have no F-theory realizations, even though there is no clear understanding of what the finite maximum $\mathrm{U}(1)$ charge allowed in F-theory constructions can be.

\subsubsection{Swampland summary}

Summarizing our discussion of the swampland and string universality, there are open questions at the level of the positivity cone, some detailed questions about which generic matter models can be realized for large gauge groups and at larger values of $b, T$, and questions about which exotic nonabelian and abelian matter representations can be realized in Ftheory. The analysis of generic matter that we have carried out here provides a useful framework in which to organize further research in these directions.

\section{Generic matter with global gauge group structure and 4D physics}

\subsection{Generic matter and the global structure of the gauge group}

In our discussions of generic matter for $\mathrm{SU}(N)$ and $\mathrm{U}(1)$ gauge groups, we have primarily assumed that there are no subtleties in the global structure of the gauge group. In particular, we have assumed that the semisimple part of the gauge algebra is associated with a simply connected gauge group and that the gauge group is connected. One can consider more complicated cases where the nonabelian part of the gauge group is not simply connected, or where the gauge group has discrete structure and is not connected. The global structure of the gauge group played a role in the last section in the discussion of swampland models with no massless matter charged under a central component of the gauge group. 
A complete analysis of these more general cases is beyond the scope of this paper, but we make some basic remarks here about a few aspects of these questions.

One useful tool in considering both low-energy 6D theories and their F-theory constructions is the Higgsing process, which can connect theories with different gauge groups. ${ }^{7}$ We have used Higgsing processes in many places in this paper to describe generic U(1) ${ }^{s}$ models from the Higgsing of generic nonabelian models with gauge groups $G_{(s)}=\mathrm{SU}(2)^{s} \times$ $\mathrm{SU}(3)^{\left(\begin{array}{c}s \\ 2\end{array}\right)}$, for $s=1,2,3, \ldots$ For nonabelian groups such as $\mathrm{SU}(N)$, a theory with generic matter content will still have generic matter content when a Higgs transition occurs from giving a vacuum expectation value (VEV) to a pair of fields in the fundamental representation, for example. In this case, the new gauge group is $\mathrm{SU}(N-1)$, and the other matter fields in the fundamental of $\mathrm{SU}(N)$, for example, decompose as $\boldsymbol{N} \rightarrow(\boldsymbol{N}-\mathbf{1})+\mathbf{1}$ under $\mathrm{SU}(N-1)$.

It is interesting to consider, however, what happens when we take an $\mathrm{SU}(N+1)$ theory with generic matter including at least one adjoint multiplet, and Higgs on an adjoint field with the $\operatorname{VEV} \operatorname{diag}(1,1, \ldots, 1,-N)$. In this case, the new gauge group is $\mathrm{SU}(N) \times$ $\mathrm{U}(1) / \mathbb{Z}_{N}$, where the discrete group $\mathbb{Z}_{N}$ has elements of the form $\operatorname{diag}(\omega, \omega, \ldots, \omega) \times \omega^{-1} \in$ $\mathrm{SU}(N) \times \mathrm{U}(1)$, with $\omega$ an $N$ th root of unity. That these elements of the discrete center of $\mathrm{SU}(N) \times \mathrm{U}(1)$ correspond to trivial elements of the original group $\mathrm{SU}(N+1)$ can be seen from the fact that the $\mathrm{SU}(N)$ factor embeds naturally in the first $N$ components of $\mathrm{SU}(N+1)$, while the $\mathrm{U}(1)$ factor is generated by $\operatorname{diag}(1,1, \ldots, 1,-N)$. Under such a Higgsing, the "bifundamental" type fields that are charged under both $\mathrm{SU}(N)$ and $\mathrm{U}(1)$ take forms that differ from the generic form when the $\mathrm{SU}(N)$ factor is simply connected. In particular, fields that take a given representation of $\mathrm{SU}(N)$ lead to $\mathrm{U}(1)$ charges that can only differ by shifts through multiples of $N$. When the $\mathrm{U}(1)$ charges are labeled in units of $1 / N$, this corresponds to unit shifts of the U(1) charges. For example, a fundamental of $\mathrm{SU}(N+1)$ leads to a fundamental of $\mathrm{SU}(N)$ with $\mathrm{U}(1)$ charge 1 , which may naturally be described as charge $1 / N$, and a single hypermultiplet that is uncharged under $\mathrm{SU}(N)$ and has $\mathrm{U}(1)$ charge $N$, naturally described as charge 1 in the units where the fundamental has charge $1 / N$. It is interesting to note that it was observed that this same shift property seems to be generic for certain F-theory realizations of $\mathrm{SU}(N) \times \mathrm{U}(1)$ gauge groups [68] (see also footnote 9), though it is not known that all F-theory models with such a gauge group can be thought of as coming from a Higgsing of a larger gauge symmetry such as $\mathrm{SU}(N+1)$. Note that there are multiple possible actions of the $\mathbb{Z}_{N}$ on the product group. For instance, when $N$ is prime, we can have a gauge group $\mathrm{SU}(N) \times \mathrm{U}(1) / \mathbb{Z}_{N}$ where the discrete group consists of elements of the form $\operatorname{diag}(\omega, \omega, \ldots, \omega) \times \omega^{-\bar{k}} \in \mathrm{SU}(N) \times \mathrm{U}(1)$, with $\omega$ an $N$ th root of unity and $\bar{k}$ the modular multiplicative inverse of $k$, which allows representations such as $\square_{k}$.

\footnotetext{
${ }^{7}$ Actually, since there is no superpotential in six dimensions, 6D supergravity theories with different numbers of tensor multiplets, different gauge groups, and different matter representations generally all live on branches of a single moduli space, connected through Higgs transitions, tensionless string transitions (which trade a tensor multiplet for 29 scalar multiplets in the simplest situations), and matter transitions [26]. For convenience, however, we often refer to branches of the theory with different massless spectra as different models or "theories." See also footnote 1.
} 


\begin{tabular}{|cc|}
\hline $\begin{array}{c}\mathrm{SU}(N) \times \mathrm{U}(1) \\
\text { Generic Matter }\end{array}$ & $\begin{array}{c}\mathrm{SU}(N) \times \mathrm{U}(1) / \mathbb{Z}_{N} \\
\text { Generic Matter }\end{array}$ \\
\hline $\mathbf{1}_{0}$ & $\mathbf{1}_{0}$ \\
$\mathbf{1}_{1}$ & $\mathbf{1}_{1}$ \\
$\mathbf{1}_{2}$ & $\mathbf{1}_{2}$ \\
$\square_{0}$ & $\square_{k / N}$ \\
$\square_{1}$ & $\square_{1+k / N}$ \\
$\square_{-1}$ & $\square_{-1+k / N}$ \\
$\square_{0}$ & $\square_{2 k / N}$ \\
$\operatorname{Adj}_{0}$ & $\operatorname{Adj}_{0}$ \\
\hline
\end{tabular}

Table 4. Generic matter representations for gauge groups $\mathrm{SU}\left(N_{1}\right) \times \mathrm{U}(1)$ and $\mathrm{SU}\left(N_{1}\right) \times \mathrm{U}(1) / \mathbb{Z}_{N}$. Here, $k \in \mathbb{Z}$ is determined by the embedding of the $\mathbb{Z}_{N}$ in the center of $\mathrm{SU}(N) \times \mathrm{U}(1)$, and we have $-N / 2<k \leq N / 2$. If $k$ is not relatively prime to $N$, then the quotient reduces to a quotient by the relevant subgroup of $\mathbb{Z}_{N}$, which is the trivial group for $k=0$.

The intuition gained from Higgsing $\mathrm{SU}(N+1) \rightarrow \mathrm{SU}(N) \times \mathrm{U}(1) / \mathbb{Z}_{N}$ naturally leads us to the question of determining the full set of generic matter for the latter gauge group. Under this Higgsing, the generic matter of $\mathrm{SU}(N+1)$ decomposes as

$$
\begin{aligned}
\square & \rightarrow \square_{1 / N}+\mathbf{1}_{1}, \\
\square & \rightarrow \square_{2 / N}+\square_{-1+1 / N}, \\
\operatorname{Adj} & \rightarrow \operatorname{Adj}_{0}+2 \times \square_{1+1 / N}+\mathbf{1}_{0},
\end{aligned}
$$

where we have chosen to label the $\mathrm{U}(1)$ charges in units of $1 / N$. The $\mathrm{AC}$ conditions tell us that we expect eight generic representations in this case, and indeed, if we add $\mathbf{1}_{2}$ to the representations on the right-hand side of eq. (5.1), this collection of representations appears to be generic. ${ }^{8}$ Note that these representations look very similar to the generic representations for $\mathrm{SU}(N) \times \mathrm{U}(1)$, in that they agree in the $N \rightarrow \infty$ limit. More generally, the discrete subgroup $\mathbb{Z}_{N}$ can be embedded in the center of $\mathrm{SU}(N) \times \mathrm{U}(1)$ in multiple ways, as noted above, and the choice of embedding determines the offset of the $\mathrm{U}(1)$ charges from those for the $\mathrm{SU}(N) \times \mathrm{U}(1)$ generic matter, as shown in table 4 .

Another set of questions involves $6 \mathrm{D}$ theories with discrete gauge groups. These have been a subject of much recent research $([65,69-73]$, see $[35,74]$ for reviews and further

\footnotetext{
${ }^{8}$ We have not proven this in complete generality, but have checked other allowed representations with small charges and confirmed that all the representations we checked can be exchanged for these generic representations with an increase in moduli space dimension.
} 
references). We make only a few brief comments here. Starting from a U(1) theory with generic matter, we have only charges $q=1,2$. Higgsing such a theory on fields of charge $q=$ 2 leads to a theory with discrete gauge group $\mathbb{Z}_{2}$ and charges $q=1$. Thus, there is a natural sense in which the discrete gauge group $\mathbb{Z}_{2}$ fits into the generic class of $6 \mathrm{D}$ supergravity theories. On the F-theory side, however, and from the point of view of counting uncharged scalar hypermultiplets, it is not clear why a $\mathbb{Z}_{2}$ discrete gauge group is more "generic" in any meaningful sense than a theory with a $\mathbb{Z}_{3}$ discrete gauge group. We leave a further exploration of these questions to future research.

\subsection{Generic matter in 4D supergravity theories and F-theory models}

From the point of view of the low-energy supergravity theory, much of the structure we have used in 6D to classify generic matter is not available in four dimensions. In particular, for $4 \mathrm{D}$ theories with $\mathcal{N}=1$ supersymmetry, there is in general a superpotential that lifts many or most of the uncharged scalar moduli fields, so the notion of generic matter as being associated with a larger number of moduli does not hold in the low-energy 4D theory; one may expect that the number of flux vacua will be exponentially larger on higher-dimensional moduli spaces [75], but the details of this are a bit harder to quantify explicitly. Furthermore, the absence of a purely gravitational anomaly in 4D means that there is not as clear an upper bound on the number of fields in the theory in $4 \mathrm{D}$, as opposed to $6 \mathrm{D}$ where eq. $(2.9 \mathrm{a})$ puts a strict bound on the number and complexity of charged and uncharged matter fields for a given gauge group.

A clearer indication perhaps for four dimensional theories is that the same geometric structures arise in constructing $4 \mathrm{D} \mathcal{N}=1$ supergravity theories from F-theory as arise in 6D. In particular, the kinds of singularities that are most generic in Weierstrass models give rise to common types of matter in $4 \mathrm{D}$ and $6 \mathrm{D}$. There are further subtleties related to fluxes, the superpotential, and chiral matter (see [35] for a recent detailed review), but there is a sense in which the same types of matter fields that are generic for $6 \mathrm{D}$ are also the most generic constructions in $4 \mathrm{D}$ F-theory models. Thus, we would expect that for $\mathrm{SU}(N)$ gauge groups coming from generic F-theory constructions without exotic singularities and associated matter, the natural representations would be the fundamental, adjoint, and two-index antisymmetric representations. Similarly, for a theory with a U(1) gauge group we would expect generically only charges $q=1,2$, unless as discussed in the previous section the $\mathrm{U}(1)$ is part of a larger group like $\mathrm{SU}(N) \times \mathrm{U}(1) / \mathbb{Z}_{N}$ that has more complicated global structure.

\subsection{Generic matter and the standard model}

The structure of generic matter seems to shed some interesting light on a long-standing question regarding the standard model of particle physics. The gauge group of the standard model is generally described as $G_{(\mathrm{SM})}=\mathrm{SU}(3)_{\mathrm{c}} \times \mathrm{SU}(2)_{\mathrm{L}} \times \mathrm{U}(1)_{Y}$, with matter fields taken by standard convention to have fractional charges under the $\mathrm{U}(1)_{Y}$ gauge field. For example, the left-handed quarks in the standard model transform in the fundamental representations of $\mathrm{SU}(3)$ and $\mathrm{SU}(2)$ with $\mathrm{U}(1)_{Y}$ charge $Y=1 / 6$, while the right-handed up quark transforms in the fundamental representation of $\mathrm{SU}(3)$ and the trivial representation of $\mathrm{SU}(2)$, with $\mathrm{U}(1)_{Y}$ charge $Y=4 / 6$. If the gauge group of the standard model observed 
in nature really has the global structure of $G_{(\mathrm{SM})}$, the natural units of charge would be such that the left-handed quarks would have a unit charge $6 Y=1$, while the right-handed electron would have charge $6 Y=-6$. Independent of charge normalization, this global structure of the gauge group would make it appear to be an accident of nature that the electron has an electromagnetic charge three times as large as the natural charge units for quarks. An alternative hypothesis is that the actual global structure of the standard model gauge group is $G=G_{(\mathrm{SM})} / \mathbb{Z}_{6}$, where the discrete $\mathbb{Z}_{6}$ has elements of the form $\operatorname{diag}\left(\omega^{2}\right) \otimes \operatorname{diag}\left(\omega^{3}\right) \otimes \omega$, with $\omega$ a sixth root of unity; all the charges of the fundamental particles in the standard model are invariant under the central $\mathbb{Z}_{6}$, so there is no empirical evidence for or against this alternative hypothesis. (In fact, there is also no empirical evidence that the $\mathrm{U}(1)_{Y}$ factor is compact instead of a non-compact $\mathbb{R}$ gauge group). See [76] for a recent analysis and further references regarding this ambiguity in the gauge group of the standard model.

From the point of view of the generic matter representations identified in this paper, the standard model gauge group without the $\mathbb{Z}_{6}$ quotient seems unnatural, in the sense that the matter fields do not fit into the generic classes of fields listed in table 2. In particular, as mentioned above the right-handed up quark has 4 units of $\mathrm{U}(1)_{Y}$ charge, the left-handed leptons have -3 units of $\mathrm{U}(1)_{Y}$ charge, etc. It is natural, therefore, to consider generic matter for the gauge group $\mathrm{SU}(3)_{\mathrm{c}} \times \mathrm{SU}(2)_{\mathrm{L}} \times \mathrm{U}(1)_{Y} / \mathbb{Z}_{6}$. The generic matter we find, along with each corresponding multiplet from the MSSM, is shown in table 5 .

Since our concrete definition of generic matter relies on the structure of $6 \mathrm{D}$ supergravity, this is the context in which we have determined the generic matter fields in table 5 . We have not proven rigorously that all possible other representations can be exchanged for the fields in this table, but we have checked this by hand for all reasonably small representations. In particular, for the gauge group of the SM, eq. (2.9a) tells us that $H \leq 285$ for any $T$ (the bound becomes more strict for larger $T$ ) in a $6 \mathrm{D}$ supergravity theory. Thus, at least for $6 \mathrm{D}$ theories with this gauge group, it suffices to check that the representations in table 5 are generic for all exchanges with representations of dimension at most 285. Carrying out this brute force check, we do find that exchanges to the representations in table 5 never decrease the number of uncharged scalars.

The upshot of this analysis is that the matter content of the MSSM consists of generic matter field types, so long as the global structure of the gauge group is $\mathrm{SU}(3)_{c} \times \mathrm{SU}(2)_{\mathrm{L}} \times$ $\mathrm{U}(1)_{Y} / \mathbb{Z}_{6}$. Note that this structure of the gauge group can arise in particular when the standard model is realized by breaking a GUT group such as $\mathrm{SU}(5), \mathrm{E}_{6}, \mathrm{E}_{7}$, or $\mathrm{E}_{8}$.

It is also interesting to consider the question of whether there are nontrivial $4 \mathrm{D}$ chiral matter models that contain only generic matter for the gauge group $\mathrm{SU}(3)_{\mathrm{c}} \times \mathrm{SU}(2)_{\mathrm{L}} \times$ $\mathrm{U}(1)_{Y}$ (without the $\mathbb{Z}_{6}$ quotient). For generic matter, we therefore consider the possible multiplicities of the fields from table 2 for a chiral theory with this gauge group. The AC conditions in 4D are linear and cubic in the hypercharge, rather than quadratic and quartic. These models can indeed have chiral matter, and so we can ask how the multiplicities of each representation must be related to those of their conjugates in order to satisfy anomaly cancellation. We note immediately that fields like the left-handed quark fields, which transform under all three gauge factors, are not among the set of generic matter fields. 


\begin{tabular}{|cc|}
\hline Generic Matter & MSSM Multiplet \\
\hline$(\mathbf{1}, \mathbf{1})_{0}$ & $\boldsymbol{N}^{c}$ \\
$(\mathbf{1}, \mathbf{1})_{1}$ & $\boldsymbol{E}^{c}$ \\
$(\square, \mathbf{1})_{2}$ & $\overline{\boldsymbol{U}^{c}}$ \\
$(\square, \mathbf{1})_{2 / 3}$ & $\overline{\boldsymbol{D}^{c}}$ \\
$(\square, \mathbf{1})_{-1 / 3}$ & \\
$(\square, \mathbf{1})_{-4 / 3}$ & $\overline{\mathbf{L}}=(\overline{\boldsymbol{N}} \quad \overline{\boldsymbol{E}}), \boldsymbol{H}_{u}, \overline{\boldsymbol{H}_{d}}$ \\
$(\mathbf{1}, \square)_{1 / 2}$ & \\
$(\text { Adj, 1 })_{3 / 2}$ & \\
$(\mathbf{1}, \text { Adj })_{0}$ & \\
$(\square, \square)_{1 / 6}$ & \\
\end{tabular}

Table 5. Generic matter representations (not including conjugates) for the gauge group $\mathrm{SU}(3)_{\mathrm{c}} \times$ $\mathrm{SU}(2)_{\mathrm{L}} \times \mathrm{U}(1)_{Y} / \mathbb{Z}_{6}$, which include all the left-handed MSSM multiplets. The generic matter for the group $\mathrm{SU}(3)_{\mathrm{c}} \times \mathrm{SU}(2)_{\mathrm{L}} \times \mathrm{U}(1)_{Y}$ does not include the MSSM multiplets.

Furthermore, the only field charged under both the $\mathrm{SU}(2)$ and $\mathrm{U}(1)_{Y}$ factors has charges $(\mathbf{1}, \square)$, so the difference between left- and right-handed multiplicities of this field must vanish by the $\mathrm{U}(1) \mathrm{SU}(2)^{2}$ anomaly. This implies that we cannot have any chiral matter that is charged under both the $\mathrm{SU}(2)_{\mathrm{L}}$ and the $\mathrm{U}(1)_{Y}$, including, e.g., a field like an "electron." There is, however, a nontrivial multi-parameter family of solutions to the complete set of $4 \mathrm{D}$ anomaly equations. Defining $\Delta x_{R}:=x_{R}-x_{\bar{R}}$, we find a family of solutions of the form

$$
\begin{gathered}
\Delta x_{(\square, \mathbf{1})_{0}}=a, \quad \Delta x_{(\mathbf{1}, \square)_{0}}=b, \quad \Delta x_{(\operatorname{Adj}, \mathbf{1})_{0}}=c, \quad \Delta x_{(\mathbf{1}, \operatorname{Adj})_{0}}=d, \\
\Delta x_{(\square, \mathbf{1})_{1}}=\Delta x_{(\square, \mathbf{1})_{-1}}=e, \quad \Delta x_{(\square, \square)_{0}}=(-a-2 e) / 2, \\
\Delta x_{(\mathbf{1}, \square)_{1}}=\Delta x_{(\mathbf{1}, \mathbf{1})_{1}}=\Delta x_{(\mathbf{1}, \mathbf{1})_{2}}=0 .
\end{gathered}
$$

If the standard model gauge group did not have a global structure with the $\mathbb{Z}_{6}$ quotient, this would appear to be the most generic type of matter we would expect from considerations of $6 \mathrm{D}$ supergravity and F-theory geometry. This would be a less phenomenologically rich world than the one we live in, however, with no chiral fields simultaneously charged under the $\mathrm{SU}(2)$ and $\mathrm{U}(1)_{Y}$ gauge factors, like the left-handed quarks and charged leptons. In the context of F-theory, we might expect to have constructions leading to either the MSSM with the gauge group having the quotient structure and the standard MSSM 
matter fields associated with generic matter from table 5, or the gauge group having the product structure without the quotient and a spectrum in the family of fields listed in eq. (5.2). The MSSM with the gauge group having no quotient taken, however, seems to involve non-generic matter and is likely disfavored by F-theory or perhaps any other approach to string compactification. ${ }^{9}$

\section{Conclusions}

In this paper, we have introduced a notion of "generic" matter representations for different gauge groups in supergravity theories. This notion is given a specific and quantitative meaning in the context of six-dimensional supergravity theories, where we define generic matter representations to be those that arise on the branches of the moduli space of largest dimension for a given gauge group when the anomaly coefficients are suitably small. This definition matches nicely with the anomaly cancellation conditions in six dimensions, and also matches with the matter representations that arise through the most direct and straightforward geometric constructions in the language of F-theory. While we use six dimensional supergravity to give a concrete definition to the notion of generic matter, the correspondence with natural constructions in F-theory suggests that this notion should also be meaningful for four-dimensional supergravity theories containing gauge groups and matter fields in various representations.

The notion of generic matter illuminates some outstanding puzzles related to $6 \mathrm{D}$ supergravity and F-theory. As we have described in section 3, the structure of generic matter clarifies what kinds of charged matter we expect in the most generic constructions of $\mathrm{F}$ theory models with multiple abelian gauge factors, and may be helpful in guiding further research on the challenging problem of explicitly constructing such models.

\footnotetext{
${ }^{9}$ Note that in [68], a swampland hypothesis was put forward regarding F-theory constructions of theories with product groups, and it was suggested that this implied that the standard model gauge group as constructed by F-theory would generally have the $\mathbb{Z}_{6}$ quotient. That argument is somewhat different from what we are saying here. The precise statement made in [68] depends upon the assumption that in a theory with a U(1) factor, the lattice of singlet charges associated with fields charged under that U(1) but no other factors determines the preferred normalization of that $\mathrm{U}(1)$ charge. For theories with only a single $\mathrm{U}(1)$ and no other gauge factors, this is a special case of the massless field swampland hypothesis mentioned in section 4.3.3. Under the more general hypothesis that this statement is true even in the presence of one or more nonabelian factors, [68] argue that in any F-theory model, all fields that transform in a given representation of the remaining gauge factors must differ by a multiple of the normalized U(1) charge. Several comments on this result and the connection to the present work: 1) This argument does not rule out in any way the existence of F-theory models with a standard model gauge group (without $\mathbb{Z}_{6}$ quotient) and spectrum composed of the fields listed in eq. (5.2), as long as there is at least one multiplet with charges $(\mathbf{1}, \mathbf{1})_{1}$ (note that this multiplicity need not vanish in $6 \mathrm{D}$ theories or for vector-like $4 \mathrm{D}$ multiplets). 2) It is not clear that the assumption quoted above is correct. In particular, consider a theory with gauge group $\mathrm{SU}(2) \times \mathrm{U}(1)$ with $x \square_{1}=0$ but $x_{\mathbf{1}_{2}}, x \square_{2} \neq 0$. This would not be allowed by this assumption. Nevertheless, there are anomaly-free models with such spectra in 6D. While no F-theory constructions for such models are known, this is likely because $\square_{2}$ is a non-generic representation and would require a fine-tuned exotic Weierstrass model; thus, we see no reason why such models cannot exist. 3) The statement that any model where the singlet $\mathrm{U}(1)$ charge can be used as a measuring stick for the massless charged fields must have a gauge group with a quotient structure is again a special case of the massless field swampland hypothesis of section 4.3.3.
} 
Generic matter also provides a useful tool for framing questions about the string swampland. As discussed in section 4 , when we restrict to $6 \mathrm{D}$ supergravity theories with a string charge lattice and positivity cone compatible with those known to arise from geometric constructions, the swampland of apparently consistent theories with no known realization in F-theory is rather limited for theories with only generic matter content. The main questions in this regard can then in large part be related to questions about exotic matter representations and the construction of Weierstrass models realizing sufficiently exotic singularities to realize these representations.

Perhaps the most interesting application of these ideas is in the context of $4 \mathrm{D}$ physics. Naively, even taking the structure of the standard model gauge group $\mathrm{SU}(3)_{\mathrm{c}} \times \mathrm{SU}(2)_{\mathrm{L}} \times$ $\mathrm{U}(1)_{Y}$ to be fixed, one could imagine consistent theories with an essentially infinite number of possible different combinations of light matter fields in different representations. Of course, the standard model is known to have one of the simplest combinations of such fields that satisfies $4 \mathrm{D}$ anomaly cancellation conditions, but the notion of generic matter gives a concrete framework that motivates why such a "simplest combination" may be favored by nature, at least in the context of a UV completion such as F-theory. In fact, as we have found here, the matter representations in the standard model are only generic if the gauge group has the global structure $\mathrm{SU}(3)_{\mathrm{c}} \times \mathrm{SU}(2)_{\mathrm{L}} \times \mathrm{U}(1)_{Y} / \mathbb{Z}_{6}$. While there is as yet no simple and direct experimental mechanism for testing this aspect of the global structure of the gauge group, this distinction is a promising sign that this kind of analysis may eventually lead us to new insights regarding important and observable features of physics beyond the standard model.

As mentioned at the end of the introduction, it is important to note that we are defining generic matter in this paper in terms of a fixed choice of gauge group. Thus, the analysis presented here represents a refinement of our understanding of the space of $6 \mathrm{D}$ supergravity theories and F-theory vacua that goes beyond the more basic question of which gauge groups are most generic or prevalent in the broader landscape of supergravity or string vacua. As also noted in the introduction, we have not here discussed strongly coupled conformal matter associated with gravitationally coupled SCFTs, which represent another important and interesting arena for study.

\section{Acknowledgments}

We would like to thank Noam Elkies, Ling Lin, Greg Moore, and Nikhil Raghuram for helpful discussions. Thanks to Yinan Wang for comments on a preliminary version of this manuscript. The authors are supported by DOE grant DE-SC00012567.

\section{A Generic matter for $\mathrm{SU}\left(N_{1}\right) \times \cdots \times \mathrm{SU}\left(N_{r}\right) \times \mathrm{U}(1)^{3}$}

In this appendix, we will prove that the matter representations given in table 2 are generic in the case of $\prod_{i} \mathrm{SU}\left(N_{i}\right) \times \mathrm{U}(1)^{3}$, in the sense that exchanges from other representations to these representations never decrease the number of uncharged scalars. We will first prove this to be true for canonical generic matter for $\mathrm{SU}(N)$ and $\mathrm{U}(1)^{3}$ individually, after which 
we can use these results in the proof for $\mathrm{SU}(N) \times \mathrm{U}(1)^{3}$ and $\prod_{i} \mathrm{SU}\left(N_{i}\right) \times \mathrm{U}(1)^{3}$. We have addressed the non-canonical generic matter types in the various sections of the main text.

\section{A.1 $\mathrm{SU}(N)$}

Note that, defining

$$
\begin{aligned}
\left(T_{12}\right)_{i j} & =\delta_{i 1} \delta_{j 1}-\delta_{i 2} \delta_{j 2}, \\
\left(T_{34}\right)_{i j} & =\delta_{i 3} \delta_{j 3}-\delta_{i 4} \delta_{j 4}, \\
\left(T_{123}\right)_{i j} & =\delta_{i 1} \delta_{j 1}+\delta_{i 2} \delta_{j 2}-2 \delta_{i 3} \delta_{j 3},
\end{aligned}
$$

the group theory coefficients $A_{R}, B_{R}, C_{R}, E_{R}$ can be computed directly via

$$
\begin{aligned}
A_{R} & =\frac{1}{2} \operatorname{tr}_{R} T_{12}^{2} \\
B_{R}+2 C_{R} & =\frac{1}{2} \operatorname{tr}_{R} T_{12}^{4}, \\
C_{R} & =\frac{3}{4} \operatorname{tr}_{R} T_{12}^{2} T_{34}^{2}, \\
E_{R} & =-\frac{1}{6} \operatorname{tr}_{R} T_{123}^{3} .
\end{aligned}
$$

In this section, we take the definition of $C_{R}$ in eq. (A.2c) at face value, so that $C_{R}=0$ for $\mathrm{SU}(2)$ and $\mathrm{SU}(3)$, while $B_{R}$ does not necessarily vanish. This is in contrast to the conventions of section 2 , where we take $B_{R}=0$ for $\mathrm{SU}(2)$ and $\mathrm{SU}(3)$, and would have $C_{R}=\frac{1}{4} \operatorname{tr}_{R} T_{12}^{4}$ for these groups.

Consider a theory with gauge group $G=\mathrm{SU}(N)$. An exchange that trades a hypermultiplet charged under the non-generic representation $R$ for some combination of hypermultiplets charged under the canonical generic matter representations in table 2 increases the number of uncharged scalars by an amount

$$
X=d+\left(\frac{3 N+1}{12}\right) g_{R}+\frac{N(N-3)}{6} C_{R}-N A_{R}
$$

where

$$
\begin{aligned}
d & =\operatorname{dim} R, \\
g_{R} & =B_{R}+2 C_{R}-A_{R} .
\end{aligned}
$$

In terms of traces, we can rewrite $X$ in the form

$$
X=d+\operatorname{tr}_{R}\left\{\frac{N}{8} T_{12}^{2}\left[T_{12}^{2}+(N-3) T_{34}^{2}-5\right]+\frac{1}{24} T_{12}^{2}\left(T_{12}^{2}-1\right)\right\} .
$$

Recall that the irreducible representations of $\mathrm{SU}(N)$ are in bijection with Young diagrams with at most $N-1$ rows. Let $\lambda$ be the partition such that the Young diagram of shape $\lambda$, which we will call $[\lambda]$, corresponds to the representation $R$. The basis elements of the representation $R$ then correspond to semistandard Young tableaux (SSYT) of shape $\lambda$ with entries in $1, \ldots, N$; we denote the set of such Young tableaux as $\operatorname{SSYT}_{N}(\lambda)$. 
Using this fact, the trace in eq. (A.5) can be evaluated as a sum over $\operatorname{SSYT}_{N}(\lambda)$ :

$$
\begin{aligned}
X=\sum_{T \in \operatorname{SSYT}_{N}(\lambda)}\{1 & +\frac{N}{8}\left(\mu_{1}-\mu_{2}\right)^{2}\left[\left(\mu_{1}-\mu_{2}\right)^{2}+\theta(N-4)(N-3)\left(\mu_{3}-\mu_{4}\right)^{2}-5\right] \\
& \left.+\frac{1}{24}\left(\mu_{1}-\mu_{2}\right)^{2}\left[\left(\mu_{1}-\mu_{2}\right)^{2}-1\right]\right\} .
\end{aligned}
$$

Here, $\mu=\left(\mu_{1}, \mu_{2}, \ldots, \mu_{N}\right)$ is the weight of the SSYT $T$, so that the entry $i$ occurs $\mu_{i}$ times in $T$, and $\theta$ is the Heaviside step function.

Our goal is to show that $X$ is always non-negative. We proceed by casework. We want to identify the SSYT for which the corresponding summand is negative, so that we can ensure they are compensated for by other positive summands and the resulting sum is never negative. For ease of notation, denote the contribution from SSYT $T$ of weight $\mu$ by $S_{\mu}$, so that

$$
\begin{aligned}
& X=\sum_{T \in \operatorname{SSYT}_{N}(\lambda)} S_{\mu} \\
& .
\end{aligned}
$$

Note that if $\mu_{1}=\mu_{2}$, then $S_{\mu}=1$.

We first consider the case that $\mu_{3}=\mu_{4}$; this also covers the cases where $N<4$, in which case there is no quartic Casimir and thus no generator $T_{34}$. In this case, we have

$$
S_{\mu}=1+\frac{k^{2}}{8}\left[N\left(k^{2}-5\right)+\frac{k^{2}-1}{3}\right]
$$

for $\mu_{1}=\mu_{2} \pm k$, which gives $S_{\mu}=1$ for $k=0, S_{\mu}=1-\frac{N}{2}$ for $k=1, S_{\mu}=\frac{3}{2}-\frac{N}{2}$ for $k=2$, and $S_{\mu}>1$ for $k>2$.

Next, we consider the case $\mu_{3}=\mu_{4} \pm 1$ and $N \geq 4$. In this case,

$$
S_{\mu}=1+\frac{k^{2}}{8}\left[N^{2}+N\left(k^{2}-8\right)+\frac{k^{2}-1}{3}\right]
$$

for $\mu_{1}=\mu_{2} \pm k$, which gives $S_{\mu}=1$ for $k=0, S_{\mu}=1+\frac{N(N-7)}{8} \geq-\frac{1}{2}$ for $k=1$, and $S_{\mu}>1$ for $k>1$.

For $\left|\mu_{3}-\mu_{4}\right| \geq 2, S_{\mu} \geq 1$ for all values of $\mu_{1}, \mu_{2}$.

Thus, there are only three types of tableaux we must consider to ensure that $X>0$ : those with $\mu_{3}=\mu_{4}($ or $N<4)$ and $\mu_{1}=\mu_{2} \pm 1$ or $\mu_{1}=\mu_{2} \pm 2$, and those with $\mu_{3}=\mu_{4} \pm 1$ and $\mu_{1}=\mu_{2} \pm 1$.

First, note that $S_{\mu} \geq 0$ for all $T$ when $N=2$, so $X \geq 0$ for $\mathrm{SU}(2)$.

For $N>2$, it will be useful to consider the collective contribution $\tilde{S}_{\mu}:=\sum_{\pi \in \mathrm{S}_{N}} S_{\pi(\mu)}$ from all diagrams of a given weight $\mu$ and all of its permutations. To see why, we briefly introduce some facts about the Kostka numbers. The number of SSYT of shape $\lambda$ and weight $\mu$ is given by the Kostka number $K_{\lambda \mu}$ [77]. A useful fact is that the Kostka number $K_{\lambda \mu}$ is invariant under permutations of the weight $\mu$ [78], i.e., $K_{\lambda \mu}=K_{\lambda \pi(\mu)}$ for any permutation $\pi \in \mathrm{S}_{N}$. Thus, every term in $\sum_{\pi \in \mathrm{S}_{N}} S_{\pi(\mu)}$ will be proportional to $K_{\lambda \mu}$. Another useful fact is that if $\mu, \mu^{\prime}$ are partitions (i.e., they are weakly decreasing) and 
$\mu \unrhd \mu^{\prime}$, then $K_{\lambda \mu^{\prime}} \geq K_{\lambda \mu}$ [79]. Here, $\unrhd$ is the dominance order, under which $\left(\mu_{1}, \ldots, \mu_{N}\right) \unrhd$ $\left(\mu_{1}^{\prime}, \ldots, \mu_{N}^{\prime}\right)$ if $\sum_{i=1}^{k} \mu_{i} \geq \sum_{i=1}^{k} \mu_{i}^{\prime}$ for all $1 \leq k \leq N$.

We now move on to consider $N>2$. We will carefully elaborate the argument for $N=3$, and then appeal to similar reasoning for $N>3$. For $N=3$, the only tableaux that contribute negatively are those with $\mu_{1}=\mu_{2} \pm 1$, which contribute $S_{\mu}=-\frac{1}{2}$. Consider the summed contribution $\tilde{S}_{\mu}$ for weight $\mu=\left(j, j+1, k^{\prime}\right)$ with fixed $j, k^{\prime}$. We know the result will be proportional to $K_{\lambda \mu}$, and the contributions $S_{\mu}$ each only depend on the relative differences of the $\mu_{i}$, so for notational simplicity we can instead refer to a "relative weight" $\mu-\mu_{1}=[0,1, k]$ for $k=k^{\prime}-j$, where we are using square brackets to indicate that the entries are taken relative to $\mu_{1}$. Furthermore, we will still account for all possible cases if we assume $k \geq 1$, because $S_{\mu}$ does not depend on the sign of the differences $\mu_{1}-\mu_{2}$ and $\mu_{3}-\mu_{4}$. The tableaux of weights $[0,1, k]$ and $[1,0, k]$ each contribute $-K_{\lambda \mu} / 2$. We have the cases $k=1, k=2$, and $k>2$. In the case $k=1$, the contribution from tableaux of weight $(1,1,0)$ is $+K_{\lambda \mu}$, as $S_{\mu}=1$ for all tableaux with $\mu_{1}=\mu_{2}$. In the case $k>2$, the contribution from tableaux of weight $(0, k, 1)$ is at least $+K_{\lambda \mu}$, as $S_{\mu} \geq 1$ for all tableaux with $\left|\mu_{1}-\mu_{2}\right|>2$. The only case in question is then $k=2$, in which case the weights $[1,2,0]$ and $[2,1,0]$ each contribute an additional $-K_{\lambda \mu} / 2$ and the weights $[0,2,1]$ and $[2,0,1]$ contribute nothing. Thus, $\tilde{S}_{[2,1,0]}=-2 K_{\lambda \mu}$ and all other contributions $\tilde{S}_{\mu}$ are non-negative. We will defer the discussion of this case to later in this section.

Similar arguments can be considered for $N>3$. Using such arguments, we can see that if the relative weight $\left[0, \mu_{2}-\mu_{1}, \ldots, \mu_{N}-\mu_{1}\right]$ contains an entry of 3 or greater, then $\tilde{S}_{\mu}$ will be non-negative, because the negative contributions will be outweighed by large positive contributions from the differences of at least three in the relevant permutations. Thus, we restrict to relative weights with only entries $0,1,2$. Again, similar arguments lead to the conclusion that the only relative weight that gives a negative $\tilde{S}_{\mu}$ is of the form $[2,1, \ldots, 1,0]$, with a single 2 , a single 0 , and all other entries 1 . Using the result above for the values of $S_{\mu}$ for a given $\mu$, we find that in this case,

$$
\tilde{S}_{[2,1, \ldots, 1,0]}=(1-N) K_{\lambda \mu}, \quad N \geq 3 .
$$

This includes the only possible trouble case we found for $N=3$.

We thus restrict our attention to relative weights of the form $[2,1, \ldots, 1,0]$ for $N \geq 3$. Our arguments thus far have only dealt with the weight $\mu$ of the tableaux, but not the shape $\lambda$. Consider a weight $\mu=(2+k, 1+k, \ldots, 1+k, k)$. Note that if the diagram $[\lambda]$ has fewer than $2+k$ columns, then $K_{\lambda \mu}=0$; in fact, $[\lambda]$ must have at least $k+1$ boxes outside of the first $k+1$ columns, as there can be at most $N-1$ rows in the Young diagram $[\lambda]$ corresponding to an irreducible representation of $\mathrm{SU}(N)$. In the case $k=0,[\lambda]$ can have a single box in the second column and none in the third. In this case, $[\lambda]$ is the adjoint representation and $\mu=(2,1, \ldots, 1,0)$. Here, we can see that the negative contribution is $(1-N) K_{\lambda \mu}=1-N$, which is exactly balanced by the positive contribution $K_{\lambda \mu^{\prime}}=N-1$ of diagrams with weight $\mu^{\prime}=(1, \ldots, 1)$. These are the only diagrams that contribute, so in this case $X=0$, as we already knew because the adjoint is one of the representations in our set of generic matter. 
In all remaining cases, there are at least two boxes outside of the first $k+1$ columns, at least one of which must be in the first row. First, consider the case that there are two additional boxes in the first row, i.e., the first row contains at least $k+3$ boxes, and consider tableaux of weight $\mu=(k, 2+k, 1+k, \ldots, 1+k)$. Because such tableaux have at least $k+3$ columns and only $k+2$ instances of the entry 2 , and also have more of each entry higher than 2 than of 1 entries, there must be at least one column with no entry 2 but containing higher entries. Thus, from each such tableau we can produce a new valid tableau of the same shape as follows: in the leftmost column that contains no 2 but does contain higher entries, we replace the lowest entry greater than 2 with a 2 . This maps each tableau of shape $\lambda$ and weight $\mu$ to a new tableau of shape $\lambda$ and weight $(k, 3+k, 1+k, \ldots, 1+k, k, 1+k, \ldots, 1+k)$, although which other entry has only $k$ instances depends on the original tableau. This map is not generally invertible, as it may not be injective; however, because all 2 entries occur in the first two rows, the map is at most two-to-one. Thus, although $K_{\lambda[0,0,3,1, \ldots, 1]} \leq K_{\lambda \mu}$ (because of the dominance order), we see that

$$
K_{\lambda \mu} \leq 2(N-2) K_{\lambda[0,0,3,1, \ldots, 1]},
$$

where the factor of $N-2$ accounts for all possible entries higher than 2 that could have been traded for an additional 2 in the above map, and the factor of 2 accounts for the map possibly being two-to-one. The negative contribution we must balance is $(1-N) K_{\lambda \mu}$, and so the more useful inequality is

$$
(N-1) K_{\lambda \mu} \leq 2(N-1)(N-2) K_{\lambda[0,0,3,1, \ldots, 1]} .
$$

Now, if we consider all tableaux of shape $\lambda$ with weight $\mu^{\prime}=(k, 3+k, k, 1+k, \ldots, 1+k)$ or any permutation thereof, their total contribution to $X$ is

$$
\frac{1}{2}(N-2)\left(7 N^{2}+9\right) K_{\lambda \mu^{\prime}}
$$

Using the inequality (A.12), we then have

$$
\begin{aligned}
& \frac{1}{2}(N-2)\left(7 N^{2}+9\right) K_{\lambda \mu^{\prime}}-(N-1) K_{\lambda \mu} \geq \frac{1}{2}(N-2)\left(7 N^{2}+9\right) K_{\lambda \mu^{\prime}} \\
&-2(N-1)(N-2) K_{\lambda \mu^{\prime}} \\
&=\frac{1}{2}(N-2)[N(7 N-4)+13] K_{\lambda \mu^{\prime}} \\
& \geq 0 .
\end{aligned}
$$

Thus, the positive contribution from such tableaux is always sufficient to balance the negative contributions from those of relative weight $[2,1, \ldots, 1,0]$ and its permutations, when $[\lambda]$ has at least $k+3$ boxes in the first row.

In the remaining cases, $[\lambda]$ must have at least two boxes in the column $k+2$, and no boxes in column $k+3$. We can then use a similar approach as above. In this case, we again consider tableaux of weight $(k, 2+k, 1+k, \ldots, 1+k)$, and note that we can map each to a new tableau by replacing with a 3 the least entry greater than 3 in the leftmost column 
that contains no 3 but does contain higher entries. This is again at most a two-to-one map. This proves that

$$
(N-1) K_{\lambda \mu} \leq 2(N-1)(N-3) K_{\lambda[0,0,2,2,1, \ldots, 1]} .
$$

Considering all tableaux of weight $\mu^{\prime}=(k, k, 2+k, 2+k, 1+k \ldots, 1+k)$ or any permutations thereof, we see that these tableaux collectively contribute

$$
\left[\frac{1}{2} N(N-1)(N-2)-2\right] K_{\lambda \mu^{\prime}}
$$

We then have

$$
\begin{aligned}
{\left[\frac{1}{2} N(N-1)(N-2)-2\right] K_{\lambda \mu^{\prime}}-(N-1) K_{\lambda \mu} } & \geq\left[\frac{1}{2} N(N-1)(N-2)-2\right] K_{\lambda \mu^{\prime}} \\
& -2(N-1)(N-3) K_{\lambda \mu^{\prime}} \\
& =\frac{1}{2}(N-2)[N(N-5)+8] K_{\lambda \mu^{\prime}} \\
& \geq 0 .
\end{aligned}
$$

This shows that the negative contribution from tableaux of relative weight $[2,1, \ldots, 1,0]$ and its permutations is compensated in all remaining cases, completing the proof. Thus, the representations presented in table 2 are generic for the gauge group $G=\mathrm{SU}(N)$.

\section{A.2 $\mathrm{U}(1)^{3}$}

Now we consider a theory with gauge group $G=\mathrm{U}(1)^{3}$. An exchange that trades a hypermultiplet charged under the non-generic representation $\left(q_{1}, q_{2}, q_{3}\right)$ for some combination of hypermultiplets charged under the canonical generic matter representations in table 2 increases the number of uncharged scalars by an amount

$$
Y=\frac{1}{16}\left[2 Q_{1}^{2}\left(Q_{2}-7\right)+Q_{2}\left(Q_{2}-6\right)+Q_{1}^{4}+4\left(Q_{22}+3 Q_{11}\right)+16\right]
$$

where

$$
\begin{aligned}
Q_{1} & =q_{1}+q_{2}+q_{3}, \\
Q_{2} & =q_{1}^{2}+q_{2}^{2}+q_{3}^{2}, \\
Q_{11} & =q_{1} q_{2}+q_{1} q_{3}+q_{2} q_{3}, \\
Q_{22} & =q_{1}^{2} q_{2}^{2}+q_{1}^{2} q_{3}^{2}+q_{2}^{2} q_{3}^{2} .
\end{aligned}
$$

This polynomial is non-negative for all integer charges $q_{i} \in \mathbb{Z}$. To see this, first note that

$$
Q_{22}+3 Q_{11}=q_{1} q_{2}\left(q_{1} q_{2}+3\right)+q_{1} q_{3}\left(q_{1} q_{3}+3\right)+q_{2} q_{3}\left(q_{2} q_{3}+3\right)
$$

has a minimum value of -2 on the integers, so $4\left(Q_{22}+3 Q_{11}\right)+16 \geq 8$ on the integers. The term $Q_{1}^{4}$ is clearly non-negative, as it is a fourth power. Thus, the only negative contributions can occur whenever $Q_{2}<7$. We can easily enumerate the possible charge combinations for which this is true: $(0,0,0),(1,0,0),(1, \pm 1,0),(1,1, \pm 1),( \pm 2,0,0),(2, \pm 1,0)$, $(2,1, \pm 1),(2,-1,-1)$, and their permutations (and conjugates). However, most of these 
representations are members of the set of generic matter in table 2, and so we already know that their exchanges do not change the number of uncharged scalars, and we can check the remaining cases by hand. We find that $Y$ is non-negative for all cases, so the representations presented in table 2 are generic for the gauge group $G=\mathrm{U}(1)^{3}$.

\section{A.3 $\mathrm{SU}(N) \times \mathrm{U}(1)^{3}$}

We now consider a theory with gauge group $\mathrm{SU}(N) \times \mathrm{U}(1)^{3}$. An exchange that trades a hypermultiplet charged under the non-generic representation $R_{\left(q_{1}, q_{2}, q_{3}\right)}$ for some combination of hypermultiplets charged under the canonical generic matter representations in table 2 increases the number of uncharged scalars by an amount

$$
P_{1}=X+d Y-d+N A_{R}\left(Q_{2}-Q_{11}\right)
$$

in terms of the quantities defined in appendices A.1 and A.2. We know already that $X, Y \geq 0$ for any $R$ and $q_{1}, q_{2}, q_{3} \in \mathbb{Z}$. To deal with the final two terms, note that $Y-1 \geq 0$ for all charge combinations other than $(1,0,0),(1, \pm 1,0),(1,1,-1),(2,0,0)$, $(2,-1,0)$, and their permutations (and conjugates). For each of these charge combinations, $Q_{2}-Q_{11} \geq 1$, and so $X-d+N A_{R}\left(Q_{2}-Q_{11}\right) \geq 0$ because $C_{R}, g_{R} \geq 0$. Thus, $P_{1} \geq 0$ for all representations $R_{\left(q_{1}, q_{2}, q_{3}\right)}$.

\section{A.4 $\mathrm{SU}\left(N_{1}\right) \times \mathrm{SU}\left(N_{2}\right) \times \mathrm{U}(1)^{3}$}

We now consider a theory with gauge group $\mathrm{SU}\left(N_{1}\right) \times \mathrm{SU}\left(N_{2}\right) \times \mathrm{U}(1)^{3}$. An exchange that trades a hypermultiplet charged under the non-generic representation $\left(R_{1}, R_{2}\right)_{\left(q_{1}, q_{2}, q_{3}\right)}$ for some combination of hypermultiplets charged under the canonical generic matter representations in table 2 increases the number of uncharged scalars by an amount

$$
P_{2}=d_{1} X_{2}+d_{2} X_{1}+d_{1} d_{2} Y+N_{1} A_{1} N_{2} A_{2}-2 d_{1} d_{2}+\left(d_{1} N_{2} A_{2}+d_{2} N_{1} A_{1}\right)\left(Q_{2}-Q_{11}\right),
$$

in terms of the quantities defined in appendices A.1 and A.2, with $X_{i}, d_{i}$, and $A_{i}:=A_{R_{i}}$ for $i=1,2$ corresponding to the respective $\mathrm{SU}\left(N_{i}\right)$ gauge factors. Using the same arguments as in the previous section, we can account for one factor of $-d_{1} d_{2}$ by absorbing it into $Y$ for most values of the $q_{i}$, or into the nonabelian terms in the remaining cases.

To account for the other factor of $-d_{1} d_{2}$, we appeal to the fact that $N A_{i} \geq d_{i}$ for all representations other than the singlet. This fact can be demonstrated using the same approach as in appendix A.1; in this case, we find that the only "trouble cases" are the relative weights of the form $\mu=[1, \ldots, 1]$, which contribute $-K_{\lambda \mu}$. Using the approach of appendix A.1, we consider the map between tableaux that replaces the entry in the box immediately to the right of the final 1 (which must exist, as there are $N(1+k)$ boxes and $1+k$ instances of entry 1 in at most $N-1$ rows, for some $k$ ) with a 1 . Unlike the earlier cases, this map is a bijection, and thus shows that

$$
K_{\lambda \mu} \leq(N-1) K_{\lambda[2,1, \ldots, 1,0]}
$$


The tableaux of weight $\mu^{\prime}=(2+k, 1+k, \ldots, 1+k, k)$ and its permutations collectively contribute $N(N+1) K_{\lambda \mu^{\prime}}$, and we then have

$$
\begin{aligned}
N(N+1) K_{\lambda \mu^{\prime}}-K_{\lambda \mu} & \geq[N(N+1)-(N-1)] K_{\lambda \mu^{\prime}} \\
& =\left(N^{2}+1\right) K_{\lambda \mu^{\prime}} \\
& \geq 0
\end{aligned}
$$

Thus, in the case that neither $R_{1}$ nor $R_{2}$ is the singlet, $N_{1} A_{1} N_{2} A_{2} \geq d_{1} d_{2}$; in the case that one of them is the singlet, say $R_{1}=\mathbf{1}$, then $d_{2} X_{1}=d_{1} d_{2}$, balancing the remaining $-d_{1} d_{2}$. Thus, $P_{2} \geq 0$ for all representations $\left(R_{1}, R_{2}\right)_{\left(q_{1}, q_{2}, q_{3}\right)}$.

\section{A.5 $\mathrm{SU}\left(N_{1}\right) \times \cdots \times \mathrm{SU}\left(N_{r}\right) \times \mathrm{U}(1)^{3}$}

Because the generic matter in table 2 only has matter charged under at most two of the nonabelian factors, we can see that the result of appendix A.4 generalizes trivially to an arbitrary number of $\mathrm{SU}(N)$ factors, completing the proof that the canonical matter in table 2 is generic for the gauge group $G=\mathrm{SU}\left(N_{1}\right) \times \cdots \times \mathrm{SU}\left(N_{r}\right) \times \mathrm{U}(1)^{3}$. By taking the all hypermultiplets to be uncharged under some or all of the $\mathrm{U}(1)$ factors, this also proves the result for $\mathrm{SU}\left(N_{1}\right) \times \cdots \times \mathrm{SU}\left(N_{r}\right) \times \mathrm{U}(1)^{s}, s \leq 3$.

\section{B Generic SU(2) matter at small $b, T>0$}

Here, we will generalize the proofs given in section 3.5.3 to higher $T$, using an approach that does not rely on the positivity cone. We will restrict our attention to $\mathrm{SU}(2)$ theories. Consider the lattice

$$
\Gamma=\operatorname{diag}(1,-1,-1, \ldots,-1),
$$

for $1 \leq T<9$, with corresponding $-a=(3,-1, \ldots,-1)$, which is fixed by the characteristic vector criterion shown in [22]. (Note that the even lattice (4.9), which was already treated in section 3.5.3, only arises at $T=1$, so we do not revisit that case here.) We wish to prove that for sufficiently small values of $b=\left(b_{0}, \ldots, b_{T}\right)$, there is always a solution of the AC conditions with only fundamentals and adjoints. We define $n=b \cdot b$ and $\gamma=-a \cdot b$.

The genus is defined as

$$
g=\frac{b \cdot(b+a)}{2}+1=\frac{n-\gamma}{2}+1
$$

This expression is given by the Riemann-Roch formula in the F-theory setting, but from the low-energy point of view, this can be taken as a definition of the quantity $g$. By taking the appropriate combination of eqs. (2.9c) and (2.9e), we can write the genus for an $\mathrm{SU}(2)$ model as

$$
g=\frac{1}{12} \sum_{R} x_{R}\left(2 C_{R}-A_{R}\right) .
$$

Thus, we can assign to each representation $R$ a genus

$$
g_{R}=\frac{2 C_{R}-A_{R}}{12}
$$


so that $g$ is the sum of the $g_{R}$ over all hypermultiplets. As mentioned in section 3.2 , an arbitrary $\mathrm{SU}(2)$ representation $\underbrace{\square \cdots \square}_{k}$ has $A_{R}=\left(\begin{array}{c}k+2 \\ 3\end{array}\right)$ and $C_{R}=\left(\begin{array}{c}k+2 \\ 3\end{array}\right) \frac{3 k(k+2)-4}{10}$. From these, we see that

$$
g_{R}=\frac{(k+3)(k+2)(k+1) k(k-1)}{120} .
$$

We see that $g_{\square}=0, g_{\square}=1$, and that the genus is strictly increasing in $k$. Thus, for a given choice of $b$, the genus must be non-negative for there to be any solutions to the AC equations, so we must have

$$
\frac{n-\gamma}{2}+1 \geq 0
$$

Now consider a choice of $b$ for which there is no valid solution (one with non-negative multiplicities) in terms of generic matter. There is a unique solution for the multiplicities $x_{1}, x_{\square}, x_{\square}$ for this $b$, and we know that $x_{\square}=g$ must be non-negative from the above argument. We further know that $x_{1} \geq 0$, because if this were not the case, then no exchanges of the form (3.8) could result in a valid solution, as they all reduce the number of uncharged scalars in exchange for larger representations. Thus, we must have $x \square<0$, by the assumption this choice of $b$ does not have a valid solution with only these three representations. This gives us the further constraint

$$
x_{\square}=8 \gamma-2 n<0 .
$$

For there to be a valid solution at all with this choice of $b$, there must be a sequence of exchanges for larger representations that will result in all non-negative multiplicities. As shown in eq. (3.8), exchanges to higher representations produce fundamentals but consume adjoints, and so there must be a sufficient number of adjoints in order for exchanges to give a positive multiplicity $x_{\square}$ without $x_{\square}$ becoming negative. From eq. (3.8), an exchange to $\underbrace{\square \cdots \square}_{k}$ produces

$$
\frac{(k+4)(k+2)(k+1) k(k-2)}{30\left(\begin{array}{c}
k+3 \\
5
\end{array}\right)}=\frac{4(k+4)(k-2)}{(k+3)(k-1)}
$$

fundamentals for every adjoint it consumes. This ratio is strictly increasing in $k$, and approaches 4 as $k \rightarrow \infty$. Thus, we must have

$$
x \square \geq-4 x \square \square
$$

in order for there to be a sufficient number of adjoints for exchanges to make all multiplicities positive. This gives us the third constraint

$$
8 \gamma-2 n \geq-4\left(\frac{n-\gamma}{2}+1\right)
$$

This constraint along with eq. (B.7) implies eq. (B.6). We see then that the range of possible $\gamma, n$ where there may be a problem with generic matter solutions is the set of integer pairs in the range

$$
\gamma \geq 0, \quad n>4 \gamma
$$


From $-a=(3,-1 \ldots,-1)$, we have

$$
n=b_{0}^{2}-\sum_{i=1}^{T} b_{i}^{2}, \quad \gamma=3 b_{0}+\sum_{i=1}^{T} b_{i} .
$$

For each fixed $T, x$, from the inequality

$$
\sum_{i=1}^{T} b_{i}^{2} \geq \frac{1}{T}\left(\sum_{i=1}^{T} b_{i}\right)^{2}
$$

we have

$$
n \leq b_{0}^{2}-\frac{1}{T}\left(\sum_{i=1}^{T} b_{i}\right)^{2}=b_{0}^{2}-\frac{\left(\gamma-3 b_{0}\right)^{2}}{T} .
$$

We see that for a fixed $T$ the resulting parabola is tangent to the line $n=4 \gamma$ when

$$
b_{0}=6+2 \sqrt{9-T} .
$$

For smaller values of $b_{0}$, the parabola lies outside the region (B.11), so there is always a solution with non-negative multiplicities of generic matter when $T<9, b_{0} \leq 6$. An explicit check shows that the first integer value of $b_{0}$ that exceeds the value in eq. (B.15) for each $T$ corresponds to a valid choice of $b$ that does not admit generic matter. For example, at $T=3, b=(11,2,2,2)$ gives $\gamma=27, n=109$, which has $n>4 \gamma$. In figure 4 , we show the parabolas $n=b_{0}^{2}-\frac{\left(\gamma-3 b_{0}\right)^{2}}{T}$ with $b_{0}=\lfloor 6+2 \sqrt{9-T}+1\rfloor$ for each $T$, and how they intersect the line $n=4 \gamma$.

For $T=9$ with the unimodular lattice (B.1), the same result holds. While from the low-energy point of view we cannot rule out the unimodular lattice $U \oplus \mathrm{E}_{8}$ at $T=9$, the only F-theory construction with this lattice uses the Enriques surface and does not admit any gauge group since the canonical class is trivial up to torsion. Similarly, any F-theory model with $T>9$ must have a larger gauge group than $\mathrm{SU}(2)$ from non-Higgsable clusters, so this argument shows that there is a non-negative generic matter spectrum for all $\mathrm{SU}(2)$ models that are not already in the swampland for other reasons.

Note that this proof does not require a choice of positivity cone, and so is strictly more powerful than the arguments given in section 3.5.3, even for the case of $T=1$.

\section{Generic matter spectra for $G=\mathrm{U}(1)^{2}$}

In this appendix, we briefly discuss generic matter for the group $G=\mathrm{U}(1)^{2}$ with general $T$.

Solving the AC equations (2.11) for the two nine-element subsets (including $(0,0)$ ) of generic $\mathrm{U}(1)^{2}$ matter that include the $(2,-1),(-1,2)$ and $(2,1),(1,2)$ pairs of charge 


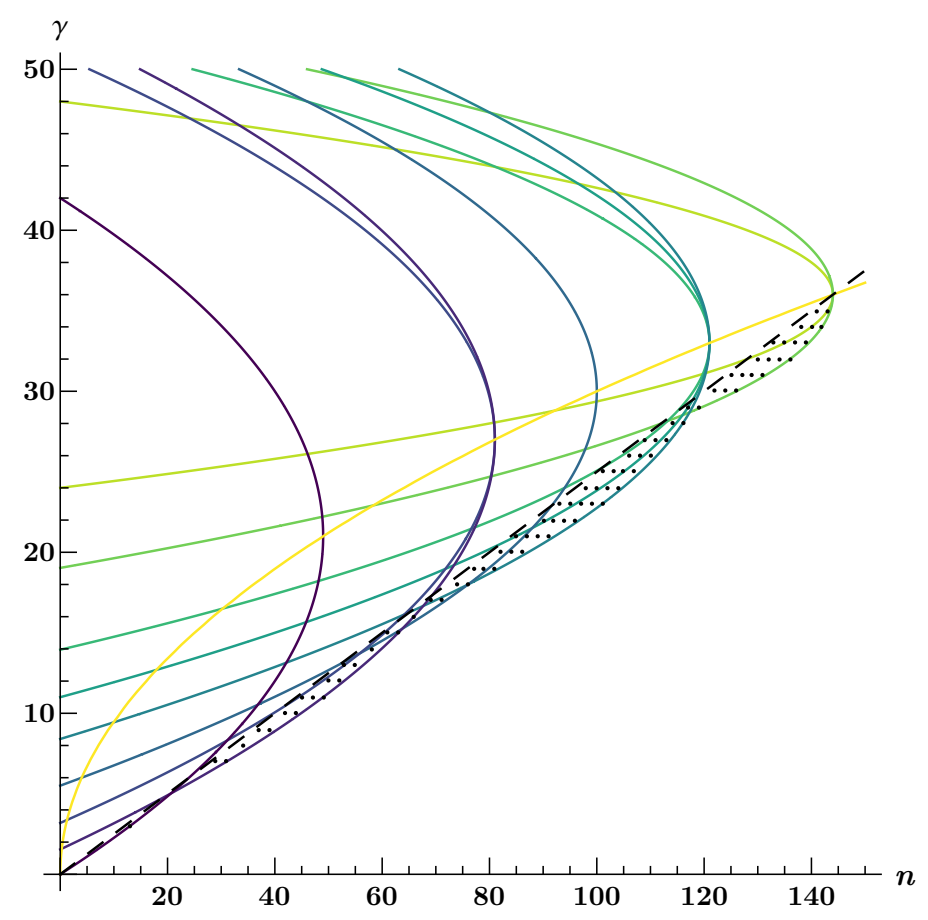

Figure 4. A plot of the parabolas $n=b_{0}^{2}-\frac{\left(\gamma-3 b_{0}\right)^{2}}{T}$ with the critical value $b_{0}=\lfloor 6+2 \sqrt{9-T}+1\rfloor$, for $T=1, \ldots, 9$. These values are $b_{0}=12,12,11,11,11,10,9,9,7$ for $T=1, \ldots, 9$, respectively. The lightest yellow curve is the parabola $n=\gamma^{2} / 9$, relevant for $T=0$, and the curves for $T=1, \ldots, 9$ appear in progressively darker colors. The dashed line is $n=4 \gamma$, the line below which there exist valid choices of anomaly coefficient that do not yield generic matter solutions. In black are points within the parabolas for $T=1, \ldots, 9$ for which there are valid solutions of the AC conditions but no solutions with generic matter.

combinations, we find that

$$
\left(\begin{array}{c}
x_{1,0} \\
x_{0,1} \\
x_{2,0} \\
x_{0,2} \\
x_{1, \pm 1} \\
x_{1, \mp 1} \\
x_{2, \pm 1} \\
x_{ \pm 1,2}
\end{array}\right)=\left(\begin{array}{cccccccc}
-8 & \pm 7 & 0 & -1 & \pm 2 & -1 & \pm \frac{3}{2} & 0 \\
0 & \pm 7 & -8 & 0 & \pm \frac{3}{2} & -1 & \pm 2 & -1 \\
\frac{1}{2} & \mp 1 & 0 & \frac{1}{4} & \mp \frac{1}{2} & 0 & 0 & 0 \\
0 & \mp 1 & \frac{1}{2} & 0 & 0 & 0 & \mp \frac{1}{2} & \frac{1}{4} \\
0 & \mp 9 & 0 & 0 & \mp \frac{3}{2} & \frac{1}{2} & \mp \frac{3}{2} & 0 \\
0 & \pm 1 & 0 & 0 & \mp \frac{1}{2} & \frac{1}{2} & \mp \frac{1}{2} & 0 \\
0 & \pm 1 & 0 & 0 & \pm \frac{1}{2} & 0 & 0 & 0 \\
0 & \pm 1 & 0 & 0 & 0 & 0 & \pm \frac{1}{2} & 0
\end{array}\right)\left(\begin{array}{c}
a \cdot b_{11} \\
a \cdot b_{12} \\
a \cdot b_{22} \\
b_{11}^{2} \\
b_{11} \cdot b_{12} \\
b_{11} \cdot b_{22}+2 b_{12}^{2} \\
b_{22} \cdot b_{12} \\
b_{22}^{2}
\end{array}\right)
$$

Similarly, solving the AC equations (2.11) for the two nine-element subsets (including $(0,0))$ of generic $\mathrm{U}(1)^{2}$ matter that include the $(2,-1),(1,2)$ and $(2,1),(-1,2)$ pairs of 
charge combinations, we find that

$$
\left(\begin{array}{c}
x_{1,0} \\
x_{0,1} \\
x_{2,0} \\
x_{0,2} \\
x_{1, \pm 1} \\
x_{1, \mp 1} \\
x_{2, \pm 1} \\
x_{\mp 1,2}
\end{array}\right)=\left(\begin{array}{cccccccc}
-8 & \pm 1 & 0 & -1 & \pm 2 & -1 & \mp \frac{3}{2} & 0 \\
0 & \mp 1 & -8 & 0 & \pm \frac{3}{2} & -1 & \mp 2 & -1 \\
\frac{1}{2} & \mp 1 & 0 & \frac{1}{4} & \mp \frac{1}{2} & 0 & 0 & 0 \\
0 & \pm 1 & \frac{1}{2} & 0 & 0 & 0 & \pm \frac{1}{2} & \frac{1}{4} \\
0 & \mp 5 & 0 & 0 & \mp \frac{3}{2} & \frac{1}{2} & \pm \frac{1}{2} & 0 \\
0 & \pm 5 & 0 & 0 & \mp \frac{1}{2} & \frac{1}{2} & \pm \frac{3}{2} & 0 \\
0 & \pm 1 & 0 & 0 & \pm \frac{1}{2} & 0 & 0 & 0 \\
0 & \mp 1 & 0 & 0 & 0 & 0 & \mp \frac{1}{2} & 0
\end{array}\right)\left(\begin{array}{c}
a \cdot b_{11} \\
a \cdot b_{12} \\
a \cdot b_{22} \\
b_{11}^{2} \\
b_{11} \cdot b_{12} \\
b_{11} \cdot b_{22}+2 b_{12}^{2} \\
b_{22} \cdot b_{12} \\
b_{22}^{2}
\end{array}\right) .
$$

By inspecting these matrices, we see that in all four cases, a good solution with nonnegative multiplicities must have $b_{12}$ satisfying

$$
\begin{aligned}
& \frac{1}{2} b_{11} \cdot\left(a+\frac{1}{2} b_{11}\right) \geq \pm b_{12} \cdot\left(a+\frac{1}{2} b_{11}\right) \geq 0 \\
& \frac{1}{2} b_{22} \cdot\left(a+\frac{1}{2} b_{22}\right) \geq \pm b_{12} \cdot\left(a+\frac{1}{2} b_{22}\right) \geq 0
\end{aligned}
$$

for the relevant choice of signs. Note that any good solution must have

$$
-a \cdot b_{11},-a \cdot b_{22}, b_{11}^{2}, b_{22}^{2}, b_{11} \cdot b_{22}+2 b_{12}^{2} \geq 0,
$$

following directly from the forms of the AC equations (2.11).

In section 3.6.3, we show that when $T=0$, there is always a non-negative generic matter spectrum when the anomaly coefficients $b$ are not too big.

Open Access. This article is distributed under the terms of the Creative Commons Attribution License (CC-BY 4.0), which permits any use, distribution and reproduction in any medium, provided the original author(s) and source are credited.

\section{References}

[1] M.B. Green, J.H. Schwarz and P.C. West, Anomaly Free Chiral Theories in Six-Dimensions, Nucl. Phys. B 254 (1985) 327 [inSPIRE].

[2] A. Sagnotti, A Note on the Green-Schwarz mechanism in open string theories, Phys. Lett. B 294 (1992) 196 [hep-th/9210127] [INSPIRE].

[3] V. Kumar and W. Taylor, A Bound on $6 D \mathcal{N}=1$ supergravities, JHEP 12 (2009) 050 [arXiv:0910.1586] [INSPIRE].

[4] V. Kumar, D.R. Morrison and W. Taylor, Global aspects of the space of $6 D \mathcal{N}=1$ supergravities, JHEP 11 (2010) 118 [arXiv: 1008.1062] [INSPIRE].

[5] W. Taylor and A.P. Turner, An infinite swampland of $\mathrm{U}(1)$ charge spectra in $6 D$ supergravity theories, JHEP 06 (2018) 010 [arXiv:1803.04447] [INSPIRE]. 
[6] L. Bhardwaj, D.R. Morrison, Y. Tachikawa and A. Tomasiello, The frozen phase of F-theory, JHEP 08 (2018) 138 [arXiv: 1805.09070] [INSPIRE].

[7] D.R. Morrison and W. Taylor, Classifying bases for $6 D$ F-theory models, Central Eur. J. Phys. 10 (2012) 1072 [arXiv:1201.1943] [inSPIRE].

[8] D.R. Morrison and W. Taylor, Non-Higgsable clusters for $4 D$ F-theory models, JHEP 05 (2015) 080 [arXiv: 1412.6112] [INSPIRE].

[9] M. Del Zotto, J.J. Heckman, A. Tomasiello and C. Vafa, 6d Conformal Matter, JHEP 02 (2015) 054 [arXiv: 1407.6359] [INSPIRE].

[10] F. Apruzzi, J.J. Heckman, D.R. Morrison and L. Tizzano, 4D Gauge Theories with Conformal Matter, JHEP 09 (2018) 088 [arXiv:1803.00582] [INSPIRE].

[11] M. Del Zotto, J.J. Heckman, D.R. Morrison and D.S. Park, 6D SCFTs and Gravity, JHEP 06 (2015) 158 [arXiv: 1412.6526] [INSPIRE].

[12] L.B. Anderson, A. Grassi, J. Gray and P.-K. Oehlmann, F-theory on Quotient Threefolds with $(2,0)$ Discrete Superconformal Matter, JHEP 06 (2018) 098 [arXiv:1801.08658] [INSPIRE].

[13] J. Halverson, C. Long and B. Sung, Algorithmic universality in F-theory compactifications, Phys. Rev. D 96 (2017) 126006 [arXiv:1706.02299] [INSPIRE].

[14] W. Taylor and Y.-N. Wang, Scanning the skeleton of the 4D F-theory landscape, JHEP 01 (2018) 111 [arXiv: 1710.11235] [INSPIRE].

[15] W. Taylor and Y.-N. Wang, The F-theory geometry with most flux vacua, JHEP 12 (2015) 164 [arXiv: 1511.03209] [INSPIRE].

[16] J. Tian and Y.-N. Wang, E-string spectrum and typical F-theory geometry, arXiv: 1811.02837 [INSPIRE].

[17] V. Kumar, D.S. Park and W. Taylor, 6D supergravity without tensor multiplets, JHEP 04 (2011) 080 [arXiv:1011.0726] [INSPIRE].

[18] A.P. Turner, Computing $\mathrm{SU}(N)$ anomaly coefficients, to appear.

[19] J. Erler, Anomaly cancellation in six-dimensions, J. Math. Phys. 35 (1994) 1819 [hep-th/9304104] [INSPIRE].

[20] D.S. Park and W. Taylor, Constraints on 6D Supergravity Theories with Abelian Gauge Symmetry, JHEP 01 (2012) 141 [arXiv:1110.5916] [INSPIRE].

[21] D.S. Park, Anomaly Equations and Intersection Theory, JHEP 01 (2012) 093 [arXiv: 1111.2351] [INSPIRE].

[22] S. Monnier, G.W. Moore and D.S. Park, Quantization of anomaly coefficients in $6 D$ $\mathcal{N}=(1,0)$ supergravity, JHEP 02 (2018) 020 [arXiv:1711.04777] [INSPIRE].

[23] M. Bershadsky and C. Vafa, Global anomalies and geometric engineering of critical theories in six-dimensions, hep-th/9703167 [INSPIRE].

[24] R. Suzuki and Y. Tachikawa, More anomaly-free models of six-dimensional gauged supergravity, J. Math. Phys. 47 (2006) 062302 [hep-th/0512019] [INSPIRE].

[25] V. Kumar, D.R. Morrison and W. Taylor, Mapping $6 D \mathcal{N}=1$ supergravities to F-theory, JHEP 02 (2010) 099 [arXiv: 0911.3393] [InSPIRE]. 
[26] L.B. Anderson, J. Gray, N. Raghuram and W. Taylor, Matter in transition, JHEP 04 (2016) 080 [arXiv: 1512.05791] [INSPIRE].

[27] N. Seiberg and W. Taylor, Charge Lattices and Consistency of 6D Supergravity, JHEP 06 (2011) 001 [arXiv:1103.0019] [INSPIRE].

[28] M. Cvetič, D. Klevers, H. Piragua and W. Taylor, General U(1) $\times$ U(1) F-theory compactifications and beyond: geometry of unHiggsings and novel matter structure, JHEP 11 (2015) 204 [arXiv: 1507.05954] [INSPIRE].

[29] C. Lüdeling and F. Ruehle, F-theory duals of singular heterotic K3 models, Phys. Rev. D 91 (2015) 026010 [arXiv: 1405.2928] [INSPIRE].

[30] M. Cvetič, J.J. Heckman and L. Lin, Towards Exotic Matter and Discrete Non-Abelian Symmetries in F-theory, JHEP 11 (2018) 001 [arXiv:1806.10594] [INSPIRE].

[31] C. Vafa, Evidence for F-theory, Nucl. Phys. B 469 (1996) 403 [hep-th/9602022] [InSPIRE].

[32] D.R. Morrison and C. Vafa, Compactifications of F-theory on Calabi-Yau threefolds. I, Nucl. Phys. B 473 (1996) 74 [hep-th/9602114] [INSPIRE].

[33] D.R. Morrison and C. Vafa, Compactifications of F-theory on Calabi-Yau threefolds. II, Nucl. Phys. B 476 (1996) 437 [hep-th/9603161] [INSPIRE].

[34] W. Taylor, TASI Lectures on Supergravity and String Vacua in Various Dimensions, arXiv:1104.2051 [INSPIRE].

[35] T. Weigand, TASI Lectures on F-theory, arXiv:1806.01854 [INSPIRE].

[36] M. Bershadsky, K.A. Intriligator, S. Kachru, D.R. Morrison, V. Sadov and C. Vafa, Geometric singularities and enhanced gauge symmetries, Nucl. Phys. B 481 (1996) 215 [hep-th/9605200] [INSPIRE].

[37] S. Katz, D.R. Morrison, S. Schäfer-Nameki and J. Sully, Tate's algorithm and F-theory, JHEP 08 (2011) 094 [arXiv: 1106.3854] [INSPIRE].

[38] Y.-C. Huang and W. Taylor, Comparing elliptic and toric hypersurface Calabi-Yau threefolds at large Hodge numbers, JHEP 02 (2019) 087 [arXiv: 1805.05907] [INSPIRE].

[39] D.R. Morrison and W. Taylor, Matter and singularities, JHEP 01 (2012) 022 [arXiv:1106.3563] [INSPIRE].

[40] D. Klevers, D.R. Morrison, N. Raghuram and W. Taylor, Exotic matter on singular divisors in F-theory, JHEP 11 (2017) 124 [arXiv:1706.08194] [INSPIRE].

[41] D. Klevers and W. Taylor, Three-Index Symmetric Matter Representations of SU(2) in F-theory from Non-Tate Form Weierstrass Models, JHEP 06 (2016) 171 [arXiv: 1604.01030] [INSPIRE].

[42] D.R. Morrison and D.S. Park, F-Theory and the Mordell-Weil Group of Elliptically-Fibered Calabi-Yau Threefolds, JHEP 10 (2012) 128 [arXiv:1208.2695] [INSPIRE].

[43] D. Klevers, D.K. Mayorga Pena, P.-K. Oehlmann, H. Piragua and J. Reuter, F-Theory on all Toric Hypersurface Fibrations and its Higgs Branches, JHEP 01 (2015) 142 [arXiv: 1408.4808] [INSPIRE].

[44] N. Raghuram, Abelian F-theory Models with Charge-3 and Charge-4 Matter, JHEP 05 (2018) 050 [arXiv:1711.03210] [INSPIRE]. 
[45] N. Raghuram and W. Taylor, Large U(1) charges in F-theory, JHEP 10 (2018) 182 [arXiv: 1809.01666] [INSPIRE].

[46] F.M. Cianci, D.K. Mayorga Peña and R. Valandro, High U(1) charges in type IIB models and their F-theory lift, JHEP 04 (2019) 012 [arXiv: 1811.11777] [INSPIRE].

[47] J. Borchmann, C. Mayrhofer, E. Palti and T. Weigand, Elliptic fibrations for $\mathrm{SU}(5) \times \mathrm{U}(1) \times \mathrm{U}(1)$ F-theory vacua, Phys. Rev. D 88 (2013) 046005 [arXiv:1303.5054] [INSPIRE].

[48] M. Cvetič, D. Klevers and H. Piragua, F-Theory Compactifications with Multiple U(1)-Factors: Constructing Elliptic Fibrations with Rational Sections, JHEP 06 (2013) 067 [arXiv: 1303.6970] [INSPIRE].

[49] M. Cvetič, A. Grassi, D. Klevers and H. Piragua, Chiral Four-Dimensional F-theory Compactifications With SU(5) and Multiple U(1)-Factors, JHEP 04 (2014) 010 [arXiv:1306.3987] [INSPIRE].

[50] J. Borchmann, C. Mayrhofer, E. Palti and T. Weigand, SU(5) Tops with Multiple U(1)s in F-theory, Nucl. Phys. B 882 (2014) 1 [arXiv:1307.2902] [INSPIRE].

[51] M. Cvetič, D. Klevers, H. Piragua and P. Song, Elliptic fibrations with rank three Mordell-Weil group: F-theory with $\mathrm{U}(1) \times \mathrm{U}(1) \times \mathrm{U}(1)$ gauge symmetry, JHEP 03 (2014) 021 [arXiv: 1310.0463] [INSPIRE].

[52] Y. Kimura, F-theory models on K3 surfaces with various Mordell-Weil ranks constructions that use quadratic base change of rational elliptic surfaces, JHEP 05 (2018) 048 [arXiv: 1802.05195] [INSPIRE].

[53] G. Martini and W. Taylor, 6D F-theory models and elliptically fibered Calabi-Yau threefolds over semi-toric base surfaces, JHEP 06 (2015) 061 [arXiv:1404.6300] [INSPIRE].

[54] D.R. Morrison, D.S. Park and W. Taylor, Non-Higgsable abelian gauge symmetry and F-theory on fiber products of rational elliptic surfaces, Adv. Theor. Math. Phys. 22 (2018) 177 [arXiv: 1610.06929] [INSPIRE].

[55] V. Kumar and W. Taylor, String Universality in Six Dimensions, Adv. Theor. Math. Phys. 15 (2011) 325 [arXiv: 0906.0987] [INSPIRE].

[56] C. Vafa, The String landscape and the swampland, hep-th/0509212 [INSPIRE].

[57] H. Ooguri and C. Vafa, On the Geometry of the String Landscape and the Swampland, Nucl. Phys. B 766 (2007) 21 [hep-th/0605264] [INSPIRE].

[58] G. Lockhart and C. Vafa, Superconformal Partition Functions and Non-perturbative Topological Strings, JHEP 10 (2018) 051 [arXiv:1210.5909] [INSPIRE].

[59] B. Haghighat, A. Iqbal, C. Kozçaz, G. Lockhart and C. Vafa, M-Strings, Commun. Math. Phys. 334 (2015) 779 [arXiv: 1305.6322] [INSPIRE].

[60] A. Gadde, B. Haghighat, J. Kim, S. Kim, G. Lockhart and C. Vafa, 6d String Chains, JHEP 02 (2018) 143 [arXiv: 1504.04614] [INSPIRE].

[61] M. Del Zotto, J. Gu, M.-X. Huang, A.-K. Kashani-Poor, A. Klemm and G. Lockhart, Topological Strings on Singular Elliptic Calabi-Yau 3-folds and Minimal 6d SCFTs, JHEP 03 (2018) 156 [arXiv: 1712.07017] [INSPIRE].

[62] M. Del Zotto and G. Lockhart, Universal Features of BPS Strings in Six-dimensional SCFTs, JHEP 08 (2018) 173 [arXiv: 1804.09694] [INSPIRE]. 
[63] S.-J. Lee, W. Lerche and T. Weigand, Tensionless Strings and the Weak Gravity Conjecture, JHEP 10 (2018) 164 [arXiv: 1808.05958] [INSPIRE].

[64] S.B. Johnson and W. Taylor, Enhanced gauge symmetry in 6D F-theory models and tuned elliptic Calabi-Yau threefolds, Fortsch. Phys. 64 (2016) 581 [arXiv: 1605. 08052] [INSPIRE].

[65] D.R. Morrison and W. Taylor, Sections, multisections and U(1) fields in F-theory, arXiv: 1404.1527 [INSPIRE].

[66] T. Banks and N. Seiberg, Symmetries and Strings in Field Theory and Gravity, Phys. Rev. D 83 (2011) 084019 [arXiv:1011.5120] [INSPIRE].

[67] D. Harlow and H. Ooguri, Symmetries in quantum field theory and quantum gravity, arXiv: 1810.05338 [INSPIRE].

[68] M. Cvetič and L. Lin, The Global Gauge Group Structure of F-theory Compactification with $\mathrm{U}(1) s$, JHEP 01 (2018) 157 [arXiv: 1706. 08521] [INSPIRE].

[69] V. Braun and D.R. Morrison, F-theory on Genus-One Fibrations, JHEP 08 (2014) 132 [arXiv: 1401.7844] [INSPIRE].

[70] C. Mayrhofer, D.R. Morrison, O. Till and T. Weigand, Mordell-Weil Torsion and the Global Structure of Gauge Groups in F-theory, JHEP 10 (2014) 16 [arXiv:1405.3656] [INSPIRE].

[71] L.B. Anderson, I. García-Etxebarria, T.W. Grimm and J. Keitel, Physics of F-theory compactifications without section, JHEP 12 (2014) 156 [arXiv:1406.5180] [INSPIRE].

[72] M. Cvetič, R. Donagi, D. Klevers, H. Piragua and M. Poretschkin, F-theory vacua with $\mathbb{Z}_{3}$ gauge symmetry, Nucl. Phys. B 898 (2015) 736 [arXiv:1502.06953] [INSPIRE].

[73] Y. Kimura, Nongeometric heterotic strings and dual F-theory with enhanced gauge groups, JHEP 02 (2019) 036 [arXiv: 1810.07657] [INSPIRE].

[74] M. Cvetič and L. Lin, TASI Lectures on Abelian and Discrete Symmetries in F-theory, PoS (TASI2017) 020 (2018) [arXiv: 1809.00012] [INSPIRE].

[75] F. Denef, Les Houches Lectures on Constructing String Vacua, Les Houches 87 (2008) 483 [arXiv:0803.1194] [INSPIRE].

[76] D. Tong, Line Operators in the Standard Model, JHEP 07 (2017) 104 [arXiv:1705. 01853] [INSPIRE].

[77] C. Kostka, Über den Zusammenhang zwischen einigen Formen von symmetrischen Funktionen, Crelle's J. 93 (1882) 89.

[78] R.P. Stanley and S. Fomin, Enumerative Combinatorics, Cambridge Studies in Advanced Mathematics, vol. 2, Cambridge University Press (1999).

[79] M. Fayers, A note on Kostka numbers, http://www.maths.qmul.ac.uk/ mf/papers/kostka.pdf (2015). 